\title{
Super-exceptional geometry: origin of heterotic M-theory and super-exceptional embedding construction of M5
}

\author{
Domenico Fiorenza, ${ }^{a}$ Hisham Sati ${ }^{b}$ and Urs Schreiber ${ }^{b, 1}$ \\ ${ }^{a}$ Dipartimento di Matematica, La Sapienza Universita di Roma, \\ Piazzale Aldo Moro 2, 00185 Rome, Italy \\ ${ }^{b}$ Mathematics, Division of Science, New York University Abu Dhabi, \\ Saadiyat Marina District, U.A.E. \\ E-mail: fiorenza@mat.uniroma1.it, hsati@nyu.edu, us13@nyu.edu
}

ABSTRACT: In the quest for the mathematical formulation of M-theory, we consider three major open problems: a first-principles construction of the single (abelian) M5-brane Lagrangian density, the origin of the gauge field in heterotic M-theory, and the supersymmetric enhancement of exceptional M-geometry. By combining techniques from homotopy theory and from supergeometry to what we call super-exceptional geometry within superhomotopy theory, we present an elegant joint solution to all three problems. This leads to a unified description of the Nambu-Goto, Perry-Schwarz, and topological Yang-Mills Lagrangians in the topologically nontrivial setting. After explaining how charge quantization of the C-field in Cohomotopy reveals D'Auria-Fré's "hidden supergroup" of 11d supergravity as the super-exceptional target space, in the sense of Bandos, for M5-brane sigma-models, we prove, in exceptional generalization of the doubly-supersymmetric superembedding formalism, that a Perry-Schwarz-type Lagrangian for single (abelian) $\mathcal{N}=(1,0)$ M5-branes emerges as the super-exceptional trivialization of the M5-brane cocycle along the super-exceptional embedding of the "half" M5-brane locus, super-exceptionally compactified on the Hořava-Witten circle fiber. From inspection of the resulting 5d super Yang-Mills Lagrangian we find that the extra fermion field appearing in super-exceptional M-geometry, whose physical interpretation had remained open, is the M-theoretic avatar of the gaugino field.

KEYwords: p-branes, M-Theory, Superspaces

ArXiv EPRINT: 1908.00042

\footnotetext{
${ }^{1}$ On leave from Czech Academy of Science, Prague, Czech Republic.
} 


\section{Contents}

1 Introduction 1

2 Perry-Schwarz Lagrangian for M5 on $S^{1} \quad 10$

3 Super-exceptional M-geometry 16

4 Super-exceptional MK6- and $\frac{1}{2} M 5$-geometry 21

5 Super-exceptional Perry-Schwarz \& Yang-Mills Lagrangians 29

6 Super-exceptional equivariance along M-theory circle 34

7 Super-exceptional M5 Lagrangian from super-exceptional embedding 42

8 Outlook 46

\section{Introduction}

An actual formulation of $M$-theory remains a fundamental open problem from physical and mathematical points of view (see [84, section 12], [69, section 2]). We had initiated in [39] a program of attacking this problem, based on universal constructions in super-homotopy theory (see [46] for review), and used this to find first-principles derivations of various aspects expected of M-theory (see [44, 47, 48]). In this paper we look to carry this further and consider the following three major sub-problems:

1. Provide a systematic construction of M5-brane Lagrangians.

2. Identify M-theory avatar degrees of freedom of the gauge field and gaugino field appearing on MO9-planes.

3. Extend exceptional M-geometry to the supergeometric setting in a natural and constructive manner.

We present a unified approach which leads to an elegant joint solution to all three at once, using the two principles, super-geometry and super-homotopy theory, explained below. 
First open problem: M5-brane Lagrangians. A widely recognized open sub-problem is the identification of the $6 \mathrm{~d}$ superconformal field theory (see [83]) on coincident M5-branes (see [77, section 3]), whose dimensional reduction to four dimensions is expected to elucidate deep aspects of non-perturbative 4-dimensional Yang-Mills theory; and not only those of theoretical interest such as $\mathcal{N}=1$ Montonen-Olive duality (see [120]), but also of profound interest in phenomenology, such as for the prediction of hadron spectra in confined quantum chromodynamics ([119, section 4], [97, 98], see [54, 93, 109]).

- Typically, it is asserted that this is an open problem only for $N \geq 2$ coincident M5branes, while the special case of a single M5-brane is well-known. Indeed, there is a non-covariant Lagrangian formulation [1, 90, 105] adapted to M5-s wrapped on the Mtheory circle fiber, as well as a covariant version at the cost of introducing an auxiliary field $[12,89]$. Both of these involved some ingenuity in their construction which makes them look somewhat baroque. Indeed, their double dimensional reduction reproduces the D4-brane Lagrangian, and hence the 5d super Yang-Mills + topological YangMills Lagrangian, only up to an intricate field redefinition [1, section 6], [2, section 6 \& appendix A].

- Such complications, already in the formulation of the base case of a theory whose expected generalization remains elusive, may indicate that the natural perspective on the problem has not been identified yet. What has been missing is a derivation of the M5-brane Lagrangian systematically from first principles of M-theory, with manifest dimensional reduction to the D4-brane.

Second open problem: Heterotic gauge enhancement. The non-perturbative completion of heterotic string theory has famously been argued $[63,64]$ to be given by the Mtheoretic completion of 11-dimensional supergravity KK-compactified on a $\mathbb{Z}_{2}$-orbifolded circle fiber, where the $\mathbb{Z}_{2}$-action on the circle has two fixed points, hence two fixed planes as an action on spacetime: the MO9-planes.

- With an actual formulation of M-theory lacking, the argument for this is necessarily indirect, and it goes as follows. Plain $11 d$ supergravity turns out to have a gravitational anomaly when considered on such MO9 boundaries, hence to be inconsistent in itself. Thus, if the putative M-theory completion indeed exists and hence is consistent, it must somehow introduce a further contribution to the total anomaly such as to cancel it. The form of that further anomaly contribution inferred this way is the same as that of a would-be field theory of charged chiral fermions on the MO9-planes, just as found in heterotic string theory.

- This suggests that if M-theory actually exists, it must include avatars of these super gauge field theory degrees of freedom appearing on MO9-branes. While many consistency checks for this assumption have been found, it remained open what the M-theoretic avatar of the heterotic gauge field actually is. In [63, 64] the 10d SYM action on the MO9s is just added by hand to that of 11d supergravity. 
Third open problem: Super-exceptional M-geometry. The Kaluza-Klein (KK) compactifications of $11 \mathrm{~d}$ supergravity on $n$-tori have a rich space of scalar moduli fields invariant under ever larger exceptional Lie groups as $n$ increases [28], reflecting just the expected duality symmetries acting on the corresponding string theories [71]. This led to the proposal [70] (see also $[9,75,87,115,116]$ ) that M-theory is an enhancement of $D=11$ supergravity to a theory of "exceptional geometry" with a "generalized tangent bundle" of the form

$$
\underbrace{T_{\text {ex }} X^{n}}_{\text {exceptional tangent bundle }}:=T X^{n} \oplus \underbrace{\wedge_{X}^{2} T^{*} X^{n}}_{\text {M2 wrapping modes }} \oplus \underbrace{\wedge_{X}^{5} T^{*} X^{n}}_{\text {M5 wrapping modes }} \oplus \cdots
$$

locally encoding wrapping modes of the M2- and the M5-brane already before KKcompactification.

- While the exceptional generalized geometry enhancements of the bosonic sector of 11d supergravity is well studied (see, e.g., [38] and references therein), the inclusion of fermionic exceptional coordinates, hence a unification of supergeometry with exceptional generalized geometry to "super-exceptional generalized geometry", had remained an open problem [24, p. 39], [25, pp. 4, 7]. Arguments were given in $[9,45,103,114]$ that the super-exceptional geometry for maximal $n=11$ is to be identified with what was called the "hidden supergroup of 11d supergravity" in $[4,10,32]$, but open questions remained. In particular, the physical meaning of

(a) the extra fermion field $\eta$ on super-exceptional spacetime (see definition 3.3 below),

(b) the parameter $s \in \mathbb{R} \backslash\{0\}$ for decompositions of the C-field (see proposition 3.5 below)

had remained open.

- It may seem that supersymmetrization is but an afterthought once the bosonic sector of exceptional geometry is understood, (e.g. [20] for $n=7$ ). But most aspects of M-theory are controlled by - and are emergent from - its local supersymmetry structure (see, e.g., $[46,111]$ ), with the bosonic sector being implied by the spin geometry, instead of the other way around. The lift of this supersymmetry first principle to exceptional generalized geometry had remained open.

The joint solution. In $[45,4.6],[103]$ we had already observed that a supersymmetric enhancement of $n=11$ exceptional M-geometry is provided by what [32] called the "hidden supergroup" of 11d supergravity. With [47, proposition 4.31], [48, proposition 4.4], it follows that this must be the correct target space for M5-brane sigma-models, as we explain in section 3. Accordingly, in section 4 we consider super-exceptional 5-brane embeddings and find in section 5 that this induces the Perry-Schwarz Lagrangian (reviewed in section 2) and, after super-exceptional equivariantization along the M-theory circle fiber introduced in section 6, the full super-exceptional M5-brane Lagrangian, in section 7. The resulting D4-brane Lagrangian with its 5d SYM+tYM Lagrangian is manifest (remark 7.5) and 
identifies the super-exceptional fermion as the M-theoretic avatar of the heterotic gauge field (remark 5.4).

Before giving more detail in the Outline of results, we recall the two foundational principles of our development:

Principle 1: Super-geometry. Despite the evident relevance of super-geometry for the foundations of M-theory, many constructions in the literature start out with the bosonic data (e.g. [1, section 2]) and relegate super-geometrization to an afterthought (e.g. [1, section 3]). Countering this tendency, the "doubly supersymmetric" approach of $[13,66]$, reviewed under the name "super-embedding approach" in [106, 107], shows that seemingly mysterious, or at least convoluted-looking, aspects of traditional constructions find their natural meaning and more elegant formulation when strictly everything is systematically internalized into super-geometry. In particular, the all-important " $\kappa$-symmetry" of super $p$ brane sigma-models, which, following [52], is traditionally imposed by hand onto the action principle, is revealed by the superembedding approach to be ([108], see [106, section 4.3], [68, section 4.3]) nothing but the super-odd-graded component of the super-worldvolume super-diffeomorphism symmetry - hence a consequence of the fundamental principle of general covariance internal to super-geometry.

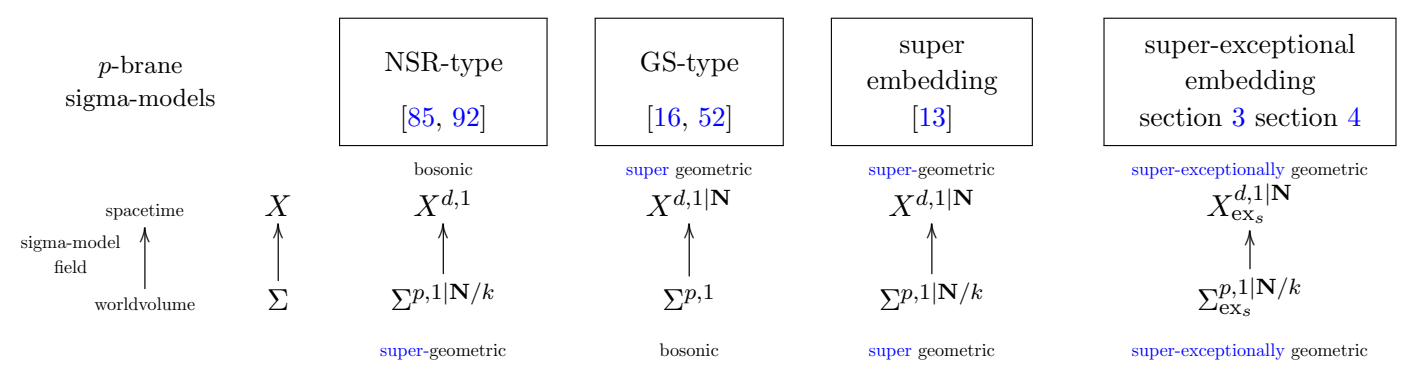

Indeed, all of the following has been systematically obtained from the superembedding approach: the equations of motion of the superstring [13, section 4] of the M2-brane [13, section 3] and of the M5-brane [66, 67], [106, 5.2], as well as the Lagrangian density of the superstring and of the M2-brane [11, 68]. But an analogous derivation of the M5-brane's Lagrangian density had remained open. Notice that it is the Lagrangian density which gives the crucial instanton contributions for these branes [15, 58].

Principle 2: homotopy theory. The gauge principle of physics — read as saying that no two things (e.g. field configuratons) are ever equal or not, but that we have to ask for gauge transformations between these, and higher order gauge-of-gauge transformations between those - is mathematically embodied in homotopy theory, these days increasingly referred to as "higher structures" (see [19, section 2] for a lightning introduction and pointers to details, and see [73] for a gentle invitation). Combining this with super-geometry yields super-homotopy theory where super-geometric $\infty$-groupoids (super$\infty$-stacks) unify super moduli spaces for higher super gauge fields with super-orbifolds 
appearing as super-spacetimes.

\begin{tabular}{|c|c|c|}
\hline & Physics & Mathematics \\
\hline & Gauge principle & Homotopy theory \\
\hline$\&$ & Pauli exclusion principle & Super-geometry \\
\hline
\end{tabular}

Homotopy theory, and more so super-homotopy theory, is extremely rich. But if, for the time being, we ignore torsion cohomology groups, homotopy theory simplifies to rational homotopy theory [91, 110] (see [53, 62], and see [46], [19, section 2] for review in our context). The main result here is that topological spaces, regarded up to rational weak homotopy equivalence, are encoded by their differential graded-commutative algebra of Sullivan differential forms, regarded up to quasi-isomorphism. If we suppress some technical fine-print (see $[19,(8)]$ for the precise statement), we may schematically write this as follows:

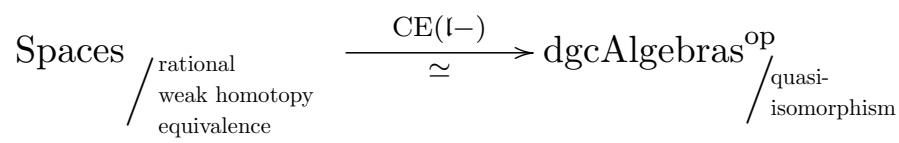

For super-homotopy theory this yields rational superspaces in rational super-homotopy theory [69, section 2] (see [46] for review). The following table shows the notation which we use, exemplified for key examples of rational super spaces (here for $X$ a topological space, we write $X_{\mathbb{R}}$ for its rationalization over $\mathbb{R}$ ):

\begin{tabular}{|c|c|c|c|}
\hline & $\begin{array}{c}\text { Rational } \\
\text { super space }\end{array}$ & $\begin{array}{c}\text { Loop } \\
\text { super } L_{\infty} \text {-algebra }\end{array}$ & $\begin{array}{c}\text { Chevalley-Eilenberg } \\
\text { super dgc-algebras } \\
\text { ("FDA"s) }\end{array}$ \\
\hline General & $X_{\mathbb{R}}$ & $\mathfrak{l} X$ & $\mathrm{CE}(\mathfrak{l} X)$ \\
\hline $\begin{array}{c}\text { Super } \\
\text { spacetime }\end{array}$ & $\mathbb{T}^{d, 1 \mid \mathbf{N}}$ & $\mathbb{R}^{d, 1 \mid \mathbf{N}}$ & $\mathbb{R}\left[\left\{\psi^{\alpha}\right\}_{\alpha=1}^{N},\left\{e^{a}\right\}_{a=0}^{d}\right] /\left(\begin{array}{l}d \psi^{\alpha}=0 \\
d e^{a}=\bar{\psi} \Gamma^{a} \psi\end{array}\right)$ \\
\hline $\begin{array}{c}\text { Eilenberg-MacLane } \\
\text { space }\end{array}$ & $K(\mathbb{R}, p+2)$ & $\mathbb{R}[p+1]$ & $\mathbb{R}\left[c_{p+2}\right] /\left(d c_{p+2}=0\right)$ \\
\hline $\begin{array}{c}\text { Odd dimensional } \\
\text { sphere }\end{array}$ & $\Upsilon_{\mathbb{R}}^{p+1} S^{1}$ & $\mathbb{R}\left[\omega_{2 k+1}\right] /\left(d \omega_{2 k+1}=0\right)$ \\
\hline $\begin{array}{c}\text { Even dimensional } \\
\text { sphere }\end{array}$ & $S_{\mathbb{R}}^{2 k+1}$ & $\mathfrak{l}\left(S^{2 k+1}\right)$ & $\mathbb{R}\left[\omega_{2 k}, \omega_{4 k-1}\right] /\left(\begin{array}{l}d \omega_{2 k}=0 \\
d \omega_{4 k-1}=-\omega_{2 k} \wedge \omega_{2 k}\end{array}\right)$ \\
\hline $\begin{array}{c}\text { M2-extended } \\
\text { super spacetime }\end{array}$ & $\widehat{\mathbb{T}^{10,1 \mid \mathbf{3} 2}}$ & $\left.\mathfrak{m} 2 S^{2 k}\right)$ & $\mathbb{R}\left[\left\{\psi^{\alpha}\right\}_{\alpha=1}^{32},\left\{e^{a}\right\}_{a=0}^{10}, h_{3}\right] /\left(\begin{array}{l}d \psi^{\alpha}=0 \\
d e^{a}=\bar{\psi} \Gamma^{a} \psi \\
d h_{3}=\mu_{\mathrm{M} 2}\end{array}\right)$ \\
\hline
\end{tabular}

(Here the reader may regard the last column to be the very definition of the second and third columns.)

One finds that a considerable amount of structures expected in M-theory emerge naturally in rational super-homotopy theory:

- On super-geometric $\infty$-groupoids, the Sullivan construction of rational homotopy theory (see, e.g., [53, 62]) unifies with higher super Lie integration [18, section 3.1] to exhibit super $L_{\infty}$-algebroids as models for rational super-homotopy theory. Their 
Chevalley-Eilenberg algebras are the "FDA"s as known in the supergravity literature $[22,32,113]$.

- Using super-homotopy theory, we had shown $[39,43]$ that the completion of the "old brane scan" to the full "brane bouquet" emerges from the superpoint $\mathbb{R}^{0 \mid 1}$ as the classification of iterated universal invariant higher central extensions.

- This process culminates [46, p. 12] in the $D=11, \mathcal{N}=1$ (hence $N=\mathbf{3 2}$ ) superMinkowski spacetime, carrying the super M2-brane cocycle $\mu_{\mathrm{M} 2}$ and, on the corresponding higher extension, the super M5-brane cocycle [39] which is the curvature of the M5 Wess-Zumino (WZ) term [12, (8)] [42] (see [46] for review):

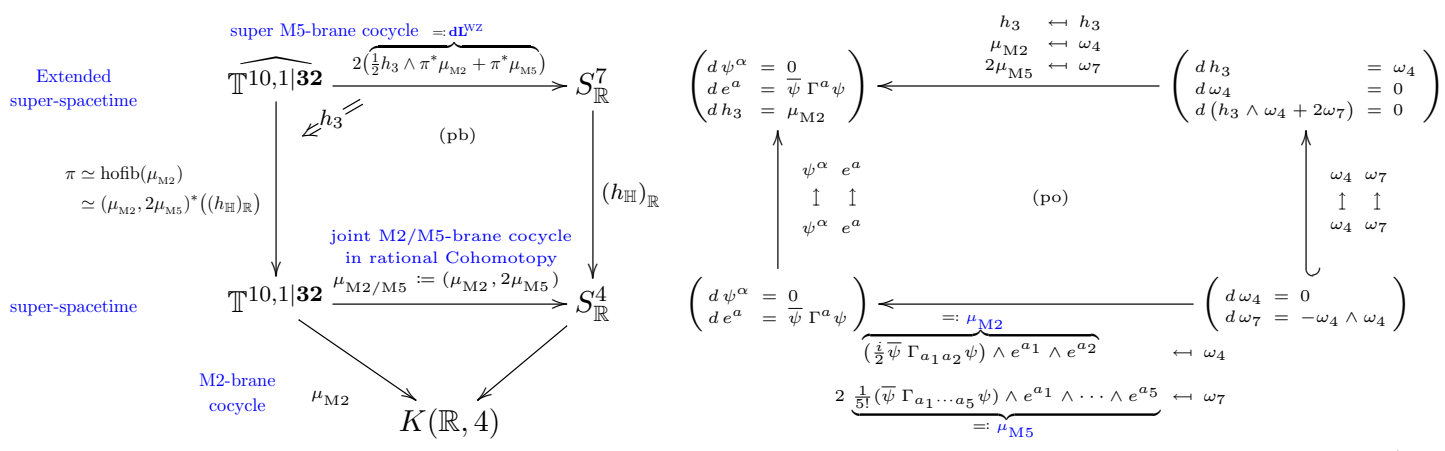

- The M2- and M5-brane cocycles unify [102, 2.5], [42] into a single non-abelian cocycle $\mu_{\mathrm{M} 2 / \mathrm{M} 5}(1.2)$ with coefficients in the rational 4-sphere, hence in rational Cohomotopy cohomology theory in degree 4.

- The Bredon-equivariant enhancement of the joint $\mu_{\mathrm{M} 2 / \mathrm{M} 5}$ cocycle to rational ADEequivariant Cohomotopy, amounts [69], via Elmendorf's theorem, to relative trivializations along super-embeddings of fixed/singular super-spacetimes:
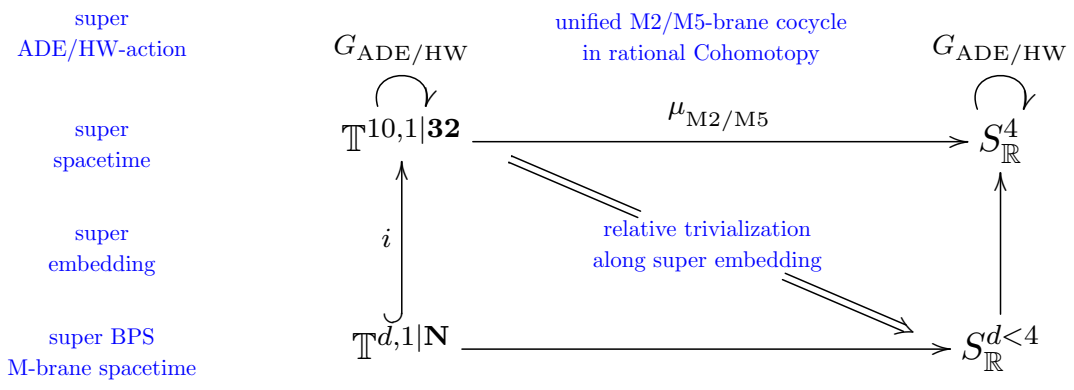

These trivializations relative to $\frac{1}{2}$-BPS super-embeddings constitute the corresponding super $p$-brane Green-Schwarz-type sigma model Lagrangian, at least for branes without gauge fields on their worldvolume [69, proposition 6.10]. We recall how this works in the case of the M2-brane. 
The M2-brane in super-homotopy theory. [69, proposition 6.10] The $\kappa$-symmetric Green-Schwarz-type Lagrangian density for the M2-brane [16] looks intricate when written out in the traditional component formulation (see [34, (2.1)], [31, (3)]), but attains a highly elegant form in a fully supergeometric formulation. Indeed, promoting the M2 worldvolume itself to a super-manifold embedded into target super-spacetime, locally of the form shown on the left of the following diagram
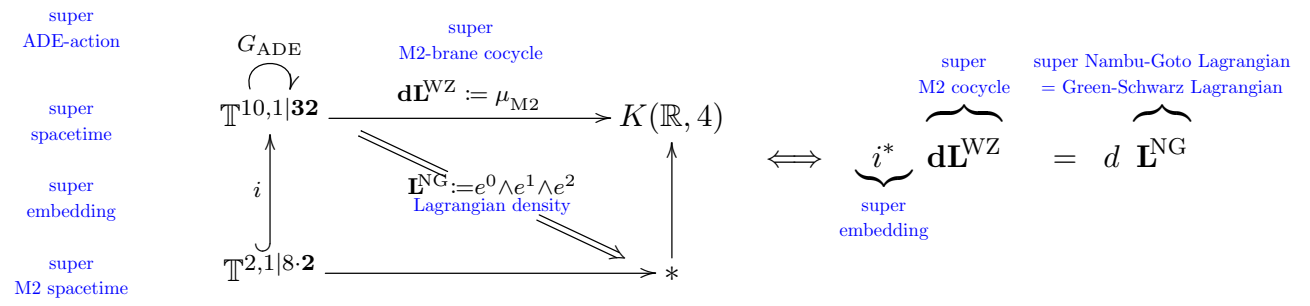

the M2-brane's Lagrangian density $\mathbf{L}^{\mathrm{NG}}$ arises simply as the super-homotopy theoretic trivialization of the M2-brane cocycle restricted along the super-embedding. Concretely, this identifies the Lagrangian with the super-volume form

$$
\begin{aligned}
\mathbf{L}^{\mathrm{NG}}:=\operatorname{svol}_{2+1}:=e^{0} \wedge e^{1} \wedge e^{2}= & \frac{1}{3 !} \epsilon_{a_{0} a_{1} a_{1}} e^{a_{0}} \wedge e^{a_{1}} \wedge e^{a_{2}} \\
& \in \Omega_{\mathrm{li}}^{\bullet}\left(\mathbb{R}^{2,1 \mid 8 \cdot \mathbf{2}}\right) \simeq \mathrm{CE}\left(\mathbb{R}^{2,1 \mid 8 \cdot \mathbf{2}}\right)
\end{aligned}
$$

on the super M2 worldvolume. Here $e^{a}:=d x^{a}+\bar{\theta} \Gamma^{a} d \theta$ denotes the vielbein 1-forms which are left-invariant "li" with respect to the translational supersymmetry action of $\mathbb{R}^{2,1 \mid 8 \cdot 2}$ on itself (see remark 3.4 below). Consequently, the super-volume form (1.5) has as bosonic component the ordinary volume form $\operatorname{vol}_{2+1} \in \Omega^{3}\left(\mathbb{R}^{2,1}\right)$, to which the fermionic components are added, ensuring overall supersymmetry-invariance of the super-volume form

$$
\mathbf{L}^{\mathrm{NG}}:=\overbrace{e^{0} \wedge e^{1} \wedge e^{2}}^{\begin{array}{c}
\text { super-volume form } \\
\text { svol }_{2}+1:=
\end{array}}=\overbrace{d x^{0} \wedge d x^{1} \wedge d x^{2}}^{\begin{array}{c}
\text { ordinary volume form } \\
=\text { vol }_{2}+1
\end{array}}+\quad \mathcal{O}(\theta \Gamma d \theta) .
$$

These fermionic correction terms, systematically obtained here simply by expanding out the super-volume form in components, constitute the otherwise intricate-looking components of the Green-Schwarz-type Lagrangian for the M2-brane, which is thereby revealed simply as the super-Nambu-Goto Lagrangian.

What had been left open in [69] is the analogous result for brane species with gauge fields on their worldvolume, notably the case of the M5-brane, which is a much richer situation (see [39]). This will be one of the main topics that we address in this paper.

Outline of results. We establish the following:

(i) In section 2 we generalize the bosonic Perry-Schwarz Lagrangian $\mathbf{L}^{\mathrm{PS}}=F \wedge \widetilde{F}$ to a coordinate-invariant expression applicable to possibly non-trivial worldvolume circle bundles.

(ii) In section 3 we recall super-exceptional M-geometry with the super-exceptional M5brane cocycle and introduce super-exceptional embedding of M-brane spacetimes. 
(iii) In section 4 we introduce specifically the super-exceptional embedding of the $\frac{1}{2} \mathrm{M} 5=$ MK6 $\cap$ MO9 brane configuration and find the super-exceptional lift of the isometry along the Hořava-Witten-circle $S_{\mathrm{HW}}^{1}$ :

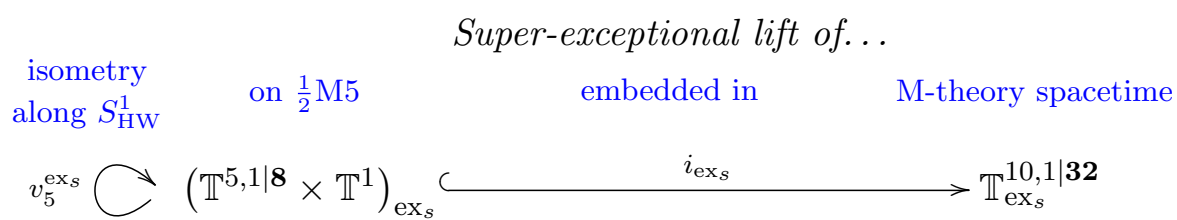
Prop. 4.4
Def. 4.6
Def. 3.3

(iv) In section 5 we find a natural super-exceptional lift of the bosonic gauge field strength with KK-modes, the bosonic Perry-Schwarz Lagrangian as well as of the topological Yang-Mills Lagrangian:

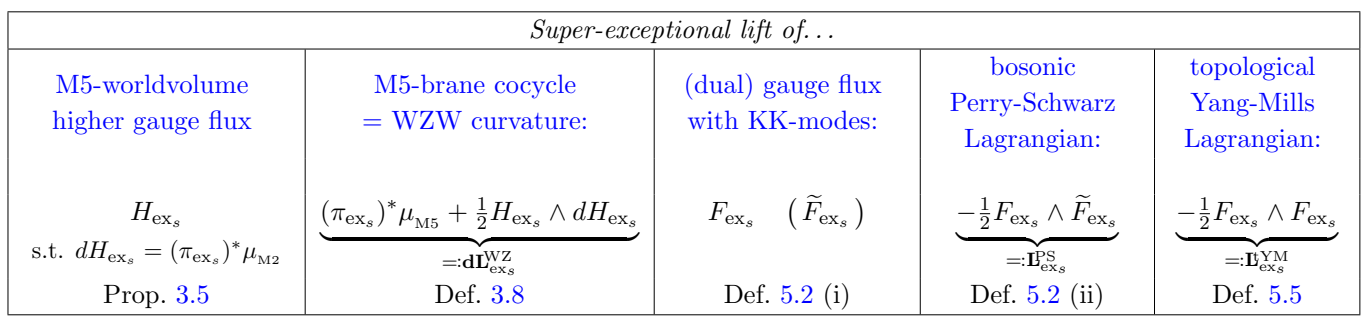

In the course of this identification we find that

(i) the putative parameter $s$ of the super-exceptional geometry is fixed to $s=-3$ (proposition 5.1 ii))

(ii) the super-exception fermion $\eta$ is the M-theory avatar of the heterotic gaugino field (proposition 5.3, remark 5.4).

Then we show (proposition 5.9) that the super-exceptional Perry-Schwarz Lagrangian arises via a super-exceptional analog of the super-embedding mechanism as a trivialization of the $S_{\mathrm{HW}}^{1}$-compactified super-exceptional M5-brane cocycle after restriction along the super-exceptional embedding of the $\frac{1}{2} \mathrm{M} 5$.

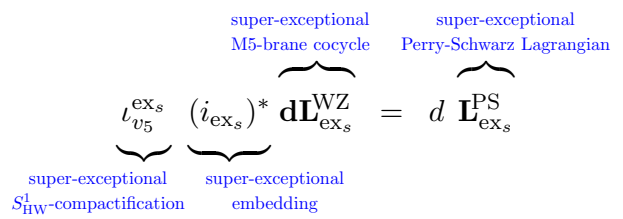

This is a partial analog for the M5-brane of the super-embedding construction of the M2-brane (1.4). To get the full statement we need not just compactify but equivariantize along $S_{\mathrm{HW}}^{1}$ :

(v) In section 6 we show that an equivariant enhancement of the super-exceptional M5cocycle with respect to super-exceptional $\Omega S_{\mathrm{HW}}^{2}$-action exists, where $\Omega S^{2}$ is the based 
loop space of the two-sphere. Furthermore, this unifies it with the super-exceptional Perry-Schwarz and the super-exceptional topological Yang-Mills Lagrangian (theorem 6.9).

To put this in perspective, we also explain (by proposition 6.6 ) how $\Omega S_{\mathrm{HW}}^{2} \rightarrow S_{\mathrm{HW}}^{1}$ refines the naive circle action by taking the super-cocycle for the little-string in $6 \mathrm{~d}$ into account. This is a $6 \mathrm{~d}$ analog to capturing the form fields in 11d M-theory via the Cohomotopical 4-sphere coefficient [42, 102], leading to a description of type IIA in ten dimensions using a refined variant of the loop space of $S^{4}$, namely the cyclic loop space [43, 44] (see [46] for overview).

(vi) In section 7 we put all the pieces together and establish (corollary 7.4) the full superexceptional embedding construction of the M5-brane Lagrangian as a sum of the super-exceptional Nambu-Goto Lagrangian and the super-exceptional Perry-Schwarz Lagrangian, the analogue of the M2 brane construction (1.4):

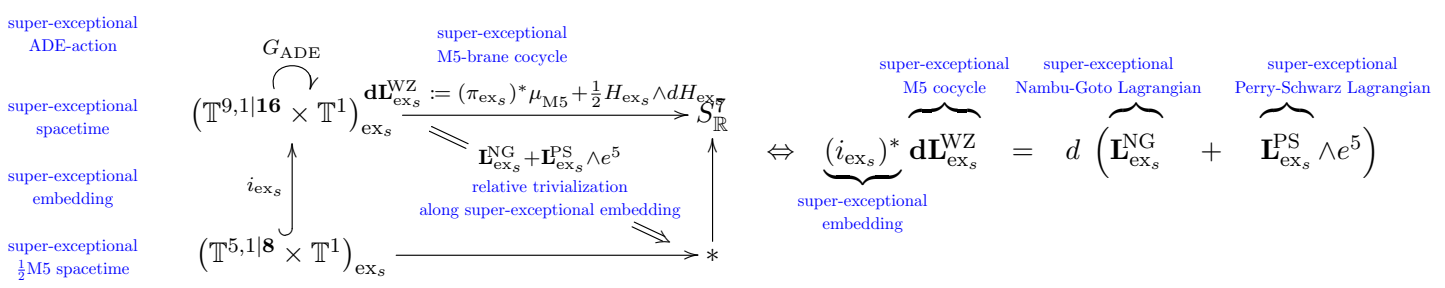

Moreover, we show (theorem 7.3) that the $\Omega S_{\mathrm{HW}}^{2}$-equivariant enhancement of the super-exceptional M5-brane cocycle, hence the homotopy-theoretic KKcompactification on the $S_{\mathrm{HW}}^{1}$-fiber, makes this super-embedding construction pick up the manifest WZ-term of the D4-brane (remark 7.5):
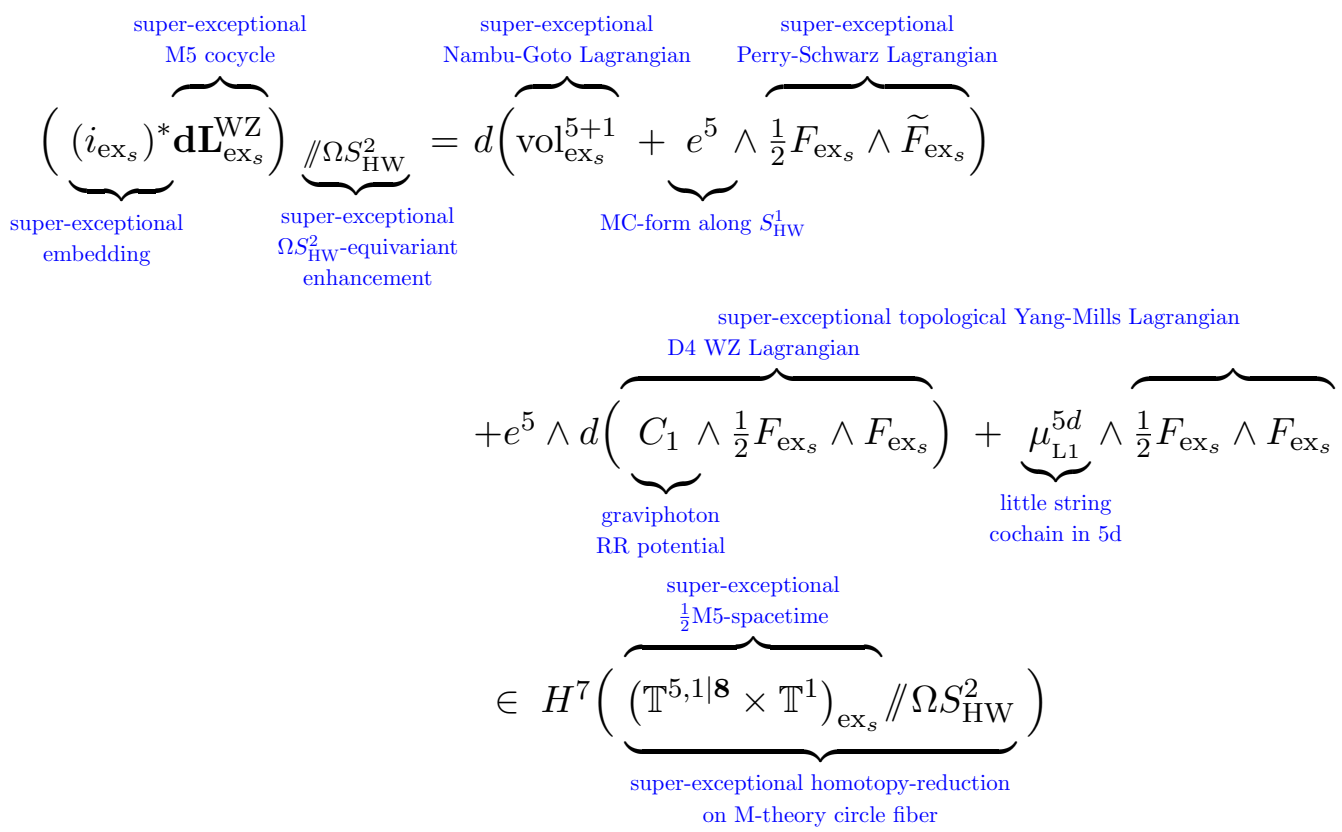
Here and in the following the double slash // denotes a homotopy quotient, which may be represented as an ordinary quotient via the Borel construction:

$$
X / / G \underset{\text { hmtpy }}{\simeq}(X \times E G) / G .
$$

Finally, we observe (remark 7.6) that there are two extensions of the compactified super-exceptional $\frac{1}{2} \mathrm{M} 5$-spacetime on which the D4 WZ-term becomes exact already before dimensional reduction: one of these implements the heterotic Green-Schwarz mechanism and the WZ-term of the heterotic NS5-brane (remark 7.8).

With this, we may sum up the whole picture elegantly in the following homotopy diagram:

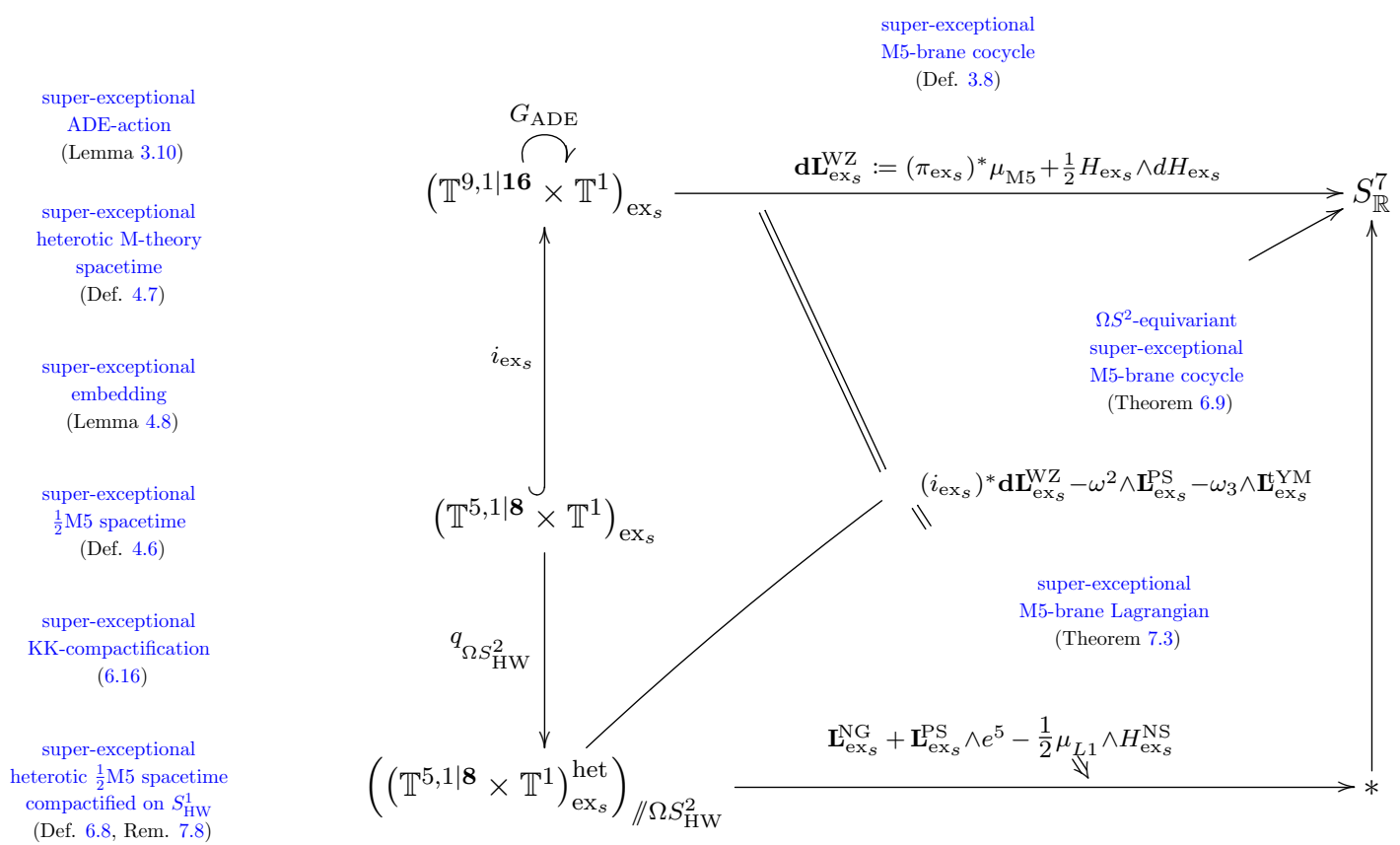

\section{$2 \quad$ Perry-Schwarz Lagrangian for M5 on $S^{1}$}

For ease of reference and in order to introduce notation needed in later sections, we review here the bosonic part of the Perry-Schwarz-Lagrangian from [90], re-cast in coordinateindependent Cartan calculus and generalized to possibly non-trivial circle fibrations. We try to bring out the logic that motivated the construction in [90], but below in section 6 and section 7 we re-derive the Perry-Schwarz Lagrangian systematically from first principles. Readers familiar with this material may want to skip this section and just follow pointers to it from the main text when needed.

The formulation of a manifestly covariant Lagrangian for the self-dual higher gauge field without further auxiliary fields in 6 dimensions (and generally in $4 k+2$-dimensions), and hence in particular for the single M5-brane sigma-model, is famously subtle, at best (see e.g. $[60,83])$. But if one considers breaking manifest Lorentz invariance to 5 dimensions, as 
befits KK compactification of the theory on a circle fiber, such as for double dimensional reduction of the M5 brane to the D4-brane, then there is a Lagrangian formulation due to Perry-Schwarz [1, 90, 105], following [61].

This "non-covariant" formulation of self-dual higher gauge theory and specifically of the M5-brane sigma-model may be covariantized by introducing an auxiliary scalar field [88] (whose gradient plays the role of the spacetime direction which gets singled out, thus promoting this choice to a dynamical field) which yields the covariant formulation of the M5-brane sigma-model $[12,89]$. This comes with a corresponding auxiliary gauge symmetry that admits a gauge fixing which recovers the non-covariant formulation, rendering the two formulations equivalent, with each "about as complicated" as the other [1, p. 3].

Worldvolume and self-duality. Let $\left(\Sigma^{6}, g\right)$ be a Lorentzian manifold of signature $(-,+,+,+,+,+)$, to be called (the bosonic body of) the worldvolume of an M5-brane. In this dimension and with this signature, corresponding to the metric $g$, the Hodge star operator on differential forms $*: \Omega^{\bullet}\left(\Sigma^{6}\right) \rightarrow \Omega^{6-\bullet}\left(\Sigma^{6}\right)$ squares to +1 . This allows for considering on a differential 3 -form

$$
H \in \Omega^{3}\left(\Sigma^{6}\right)
$$

the condition that it be self-dual

$$
H=* H .
$$

We will assume that $H$ is exact and pick a trivializing 2-form

$$
B \in \Omega^{2}\left(\Sigma^{6}\right) \quad \text { such that } \quad H=d B .
$$

Compactification on $\boldsymbol{S}^{\mathbf{1}}$. Consider then on the worldvolume $\Sigma^{6}$ the structure of an $S^{1}=\mathrm{U}(1)$-principal bundle

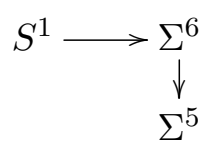

We write $v_{5} \in \Gamma\left(T \Sigma^{6}\right)$ for the vector field which encodes the infinitesimal $S^{1}$-action, hence the derivative of the circle action $\mathrm{U}(1) \times \Sigma^{6} \stackrel{\rho}{\longrightarrow} \Sigma^{6}$ at the neutral element, along a chosen basis element $t \in T_{e}(\mathrm{U}(1)) \simeq \mathfrak{u}(1) \simeq \mathbb{R}$ :

$$
v_{5}: \Sigma^{6} \simeq\{(e, t)\} \times \Sigma^{6} \longleftrightarrow T S^{1} \oplus_{\Sigma^{6}} T \Sigma^{6} \simeq T\left(S^{1} \times \Sigma^{6}\right) \longrightarrow d \rho \longrightarrow T \Sigma^{6} .
$$

Accordingly, we write

$$
\mathcal{L}_{v_{5}}:=\underbrace{\left[d, \iota_{v_{5}}\right]}_{d \circ \iota_{v_{5}}+\iota_{v_{5}} \text { od }}: \Omega^{\bullet}\left(\Sigma^{6}\right) \longrightarrow \Omega^{\bullet}\left(\Sigma^{6}\right)
$$

for the Lie derivative of differential forms along the vector field (2.5), where $d$ denotes the de Rham differential and where under the brace we are using Cartan's magic formula.

Next, consider an Ehresmann connection on the $S^{1}$-bundle (2.4), hence a differential 1-form which satisfies the Ehresmann conditions in that it is normalized and invariant:

$$
\theta^{5} \in \Omega^{1}\left(\Sigma^{6}\right) \quad \text { such that } \quad \iota_{v_{5}} \theta^{5}=1 \quad \text { and } \quad \mathcal{L}_{v_{5}} \theta^{5}=0 .
$$


Here on the left we have the operation of contracting differential forms with vector fields, and on the right we have the Lie derivative from (2.6). So, in particular, the composition

$$
\theta^{5} \wedge \circ \iota_{v_{5}}: \Omega^{\bullet}\left(\Sigma^{6}\right) \longrightarrow \Omega^{\bullet}\left(\Sigma^{6}\right)
$$

is a projection operator: $\theta^{5} \wedge \iota_{v_{5}} \circ \theta^{5} \wedge \iota_{v_{5}}=\theta^{5} \wedge \iota_{v_{5}}$. The complementary projection is that onto horizontal differential forms with respect to the bundle structure (2.4):

$$
(-)^{\text {hor }}:=\left(\mathrm{id}-\theta^{5} \wedge \mathrm{o}_{v_{5}}\right): \Omega^{\bullet}\left(\Sigma^{6}\right) \longrightarrow \Omega^{\bullet}\left(\Sigma^{6}\right) .
$$

Observe that:

Lemma 2.1 (Horizontal vs. vertical differential). If the Ehresmann connection (2.7) on the $S^{1}$ bundle is flat, in that

$$
d \theta^{5}=0
$$

then for any differential form $\omega \in \Omega^{\bullet}\left(\Sigma^{6}\right)$ we have that the vertical component of the differential of its horizontal component (2.9) is the vertical component of its full differential:

$$
\theta^{5} \wedge d\left(\omega^{\text {hor }}\right)=\theta^{5} \wedge d \omega
$$

Proof. By direct computation, we have:

$$
\begin{aligned}
\theta^{5} \wedge d\left(\omega^{\text {hor }}\right) & =\theta^{5} \wedge d\left(\omega-\theta^{5} \wedge \iota_{v_{5}} \omega\right) \\
& =\theta^{5} \wedge d \omega-\theta^{5} \wedge d\left(\theta^{5} \wedge \iota_{v_{5}} \omega\right) \\
& =\theta^{5} \wedge d \omega-\underbrace{\theta^{5} \wedge\left(d \theta^{5}\right) \wedge \iota_{v_{5}} \omega}_{=0} .
\end{aligned}
$$

Finally, we require the vector field $v_{5}$ from (2.5) to be a spacelike isometry. This means that it interacts with the Hodge star operator as

$$
* \circ \iota_{v_{5}}=-\theta^{5} \wedge \circ *: \Omega^{3}\left(\Sigma^{6}\right) \longrightarrow \Omega^{4}\left(\Sigma^{6}\right) .
$$

Self-duality after $\boldsymbol{S}^{\mathbf{1}}$-compactification. We introduce notation for the contraction of the 3 -form $H$ and its Hodge dual with the vector field $v_{5}(2.5)$ as follows (to be called the "compactified fields", a notation that follows [90, (5), (6)]):

$$
\mathcal{F}:=\iota_{v_{5}} H, \quad \tilde{H}:=\iota_{v_{5}} * H .
$$

With this, we get the following immediate but crucially important re-formulation of the self-duality condition after $S^{1}$-compactification (extending [90, (8)]):

Lemma 2.2 (Self-duality after $S^{1}$-compactification.). Given an $S^{1}$-bundle structure (2.4) on the worldvolume $\Sigma^{6}$ and any choice of Ehresmann connection (2.7), the self-duality condition (2.2) is equivalently expressed in terms of the compactified fields (2.13) as:

$$
H=* H \quad \Leftrightarrow \quad \mathcal{F}=\tilde{H} .
$$


Proof. We have the following chain of equivalences:

$$
\begin{aligned}
H=* H & \Longleftrightarrow\left(\begin{array}{c}
\iota_{v_{5}} H=\iota_{v_{5}} * H \\
\text { and } \theta^{5} \wedge H=\theta^{5} \wedge * H
\end{array}\right) \\
& \Longleftrightarrow \iota_{v_{5}} H=\iota_{v_{5}} * H \\
& \Longleftrightarrow \mathcal{F}=\tilde{H} .
\end{aligned}
$$

Here the first step is decomposition into horizontal and vertical components (2.9), the second step uses the isometry property (2.12) to conclude that the two resulting component equations are equivalent to each other. The last step identifies the compactified fields (2.13).

The gauge field. The contraction of the vector field $v_{5}$ from (2.5) with the 2 -form potential $B$ from (2.3) defines the 1-form potential

$$
A:=-\iota_{v_{5}} B \text {. }
$$

Hence we get a decomposition of the 2-form as

$$
B=A \wedge \theta^{5}+B^{\text {hor }},
$$

where on the right we have the horizontal component of $B$ according to (2.9). We say that the 2-flux density encoded by $B$ is the horizontal component of the exterior differential of this vector potential

$$
F:=(d A)^{\text {hor }} .
$$

We will find in a moment that this is the 5d field strength with all higher KK-modes still included, but it is most convenient here (and in all of the following to just call it " $F$ " already in the $6 \mathrm{~d}$ compactification before passing to KK zero-modes. With this we have (cf. $[90,(5)])$ :

Lemma 2.3 (Shifted 2-flux). The 2-flux density $F$ from (2.17) equals the compactified field $\mathcal{F}$ from (2.13) up to the Lie derivative (2.6) of the horizontal component (2.9) of the 2-form (2.3):

$$
F=\mathcal{F}-\mathcal{L}_{v_{5}} B^{\text {hor }}
$$

Proof. We compute as follows:

$$
\begin{aligned}
\mathcal{F} & :=\iota_{v_{5}} H \\
& =\iota_{v_{5}} d B \\
& =-d \iota_{v_{5}} B+\left[\iota_{v_{5}}, d\right] B \\
& =d A+\mathcal{L}_{v_{5}} B \\
& =\underbrace{(d A)^{\text {hor }}}_{=F}+\underbrace{\theta^{5} \wedge \iota_{v_{5}} d A}_{=\theta^{5} \wedge \mathcal{L}_{v_{5}} A}+\mathcal{L}_{v_{5}} B^{\text {hor }}+\underbrace{\mathcal{L}_{v_{5}} \theta^{5} \wedge \iota_{v_{5}} B}_{-\theta^{5} \wedge \mathcal{L}_{v_{5}} A} \\
& =F+\mathcal{L}_{v_{5}} B^{\text {hor }} .
\end{aligned}
$$


Here the first step is the definition (2.13), while the second step is (2.3). The fourth step uses the definition (2.15) of the vector potential and identifies the Lie derivative (2.6). The fifth step applies vertical/horizontal decomposition (2.9) to both summands and uses (2.17) under the first brace and the expressions (2.7) and (2.15) under the third brace; while under the second brace it uses Cartan's formula (2.6), observing that $\iota_{v_{5}} A=0$ by definition (2.15) and by nilpotency of the contraction operation. The last step notices that this makes the second and fourth summands cancel each other.

With lemma 2.3, the self-duality condition (2.2) in the equivalent form (2.14) after $S^{1}$-compactification says that the combination $\widetilde{H}-\mathcal{L}_{v_{5}} B^{\text {hor }}$ is horizontally exact:

$$
H=* H \quad \Leftrightarrow \quad \widetilde{H}-\mathcal{L}_{v_{5}} B^{\text {hor }}=\underbrace{(d A)^{\text {hor }}}_{=F} .
$$

Weak self-duality and PS equations of motion. In summary, we have the following implication of self-duality after $S^{1}$-compactification (extending [90, (16)]):

Proposition 2.4 (Self-duality for flat circle bundles). If the worldvolume $\Sigma^{6}$ is equipped with an $S^{1}$-principal bundle structure (2.4) which is flat (2.10), then the self-duality condition $H=* H$ from (2.2), in its equivalent incarnation on compactified fields (2.20) implies the following differential equation:

$$
\theta^{5} \wedge d\left(\tilde{H}-\mathcal{L}_{v_{5}} B^{\text {hor }}\right)=0 .
$$

By lemma 2.1, equation (2.21) may be understood as expressing "self-duality up to horizontally exact terms". The proposal of [90] is to regard (2.21), which is second order as a differential equation for $B$, as the defining equation of motion for a self-dual field on $\Sigma^{6}$ compactified on $S^{1}$. Given this, one is led to finding a Lagrangian density whose Euler-Lagrange equation is the self-duality equation.

The PS Lagrangian density. As in [90, (17)], we say:

Definition 2.5 (Bosonic PS Lagrangian). The bosonic Perry-Schwarz-Lagrangian for a 2 -form field (2.3) on a worldvolume $\Sigma^{6}$ compactified on a flat (2.10) $S^{1}$-bundle (2.4) is:

$$
B \longmapsto \mathbf{L}^{\mathrm{PS}} \wedge \theta^{5}:=\frac{1}{2}\left(\mathcal{L}_{v_{5}} B^{\text {hor }}-\tilde{H}\right) \wedge * \tilde{H}
$$

Remark 2.6 (Equivalent descriptions). (i) Equivalently, using the isometry property (2.12) the bosonic PS Lagrangian (2.22) reads

$$
\begin{aligned}
\mathbf{L}^{\mathrm{PS}} \wedge \theta^{5} & =-\frac{1}{2}\left(\tilde{H}-\mathcal{L}_{v_{5}} B^{\text {hor }}\right) \wedge H \wedge \theta^{5} \\
& =-\frac{1}{2}\left(\iota_{v_{5}} * H-\mathcal{L}_{v_{5}} B^{\text {hor }}\right) \wedge H \wedge \theta^{5},
\end{aligned}
$$

where in the second line we inserted the expression for $\widetilde{H}$ from (2.13); (ii) and if the self-duality condition (2.2) is imposed, the bosonic PS Lagrangian (2.23) becomes

$$
\mathbf{L}^{\mathrm{PS}} \wedge \theta^{5}=-\frac{1}{2}\left(\iota_{v_{5}} H-\mathcal{L}_{v_{5}} B^{\text {hor }}\right) \wedge H \wedge \theta^{5} \quad \text { if } H=* H .
$$


At this point, as in $[90,(16)]$, the bosonic PS Lagrangian of definition 2.5 is motivated as the evident choice that makes the following proposition 2.7 true. Below in section 5 , we find a deeper origin of the Lagrangian (in the case that self-duality is imposed).

Proposition 2.7 (EOMs of the bosonic PS Lagrangian). The Euler-Lagrange equation corresponding to the PS Lagrangian $\mathbf{L}^{\mathrm{PS}} \wedge \theta^{5}$ (2.22) is the weak self-duality equation (2.21).

Proof. We may evidently regard the PS Lagrangian as the quadratic part of the following bilinear form on differential 2-forms with values in differential 6-forms:

$$
\left(B, B^{\prime}\right) \longmapsto-\frac{1}{2}\left(\iota_{v_{5}} * H-\mathcal{L}_{v_{5}} B^{\text {hor }}\right) \wedge d B^{\prime} \wedge \theta^{5} .
$$

Observe then that this bilinear form is symmetric up to a total differential: the first summand is strictly symmetric, as it is the standard Hodge pairing, while for the second summand symmetry up to a total derivative is established by a local integration by parts. Together these imply that the variational Euler-Lagrange derivative of the PS Lagrangian is twice the result of varying just the second factor of $B$ :

$$
\begin{aligned}
\delta \mathbf{L}^{\mathrm{PS}} \wedge \theta^{5} & =-\left(\iota_{v_{5}} * H-\mathcal{L}_{v_{5}} B^{\text {hor }}\right) \wedge d(\delta B) \wedge \theta^{5} \\
& =-\left(\theta^{5} \wedge d\left(\iota_{v_{5}} * H-\mathcal{L}_{v_{5}} B^{\text {hor }}\right)\right) \wedge(\delta B) .
\end{aligned}
$$

Hence the vanishing of the variational derivative is equivalent to (2.21).

Reduction to 5d Maxwell theory. Consider finally the special case of Kaluza-Klein compactification/double dimensional reduction, where

$$
\mathcal{L}_{v_{5}} B^{\text {hor }}=0 \quad \text { for KK-reduction to D4. }
$$

In this case, expression (2.19) reduces to

$$
\iota_{v_{5}} H=F \quad \text { if } \quad \mathcal{L}_{v_{5}} B^{\text {hor }}=0 .
$$

Hence, with self-duality ([90, above (16)]), we have

$$
H=F \wedge \theta^{5}+*_{5} F,
$$

where now $*_{5}: \Omega^{\bullet}\left(\Sigma^{5}\right) \rightarrow \Omega^{5-\bullet}\left(\Sigma^{5}\right)$ is the Hodge star operator on the base 5-manifold. Consequently, we have extension of [90, above (16)] to the topologically nontrivial setting:

Proposition 2.8 (5d Abelian Yang-Mills from Perry-Schwarz). The $S^{1}$-dimensional reduction of the PS Lagrangian (2.24) is the Lagrangian of $5 d$ Maxwell theory for the vector potential A from (2.15):

$A \longmapsto \mathbf{L}^{\mathrm{PS}} \wedge \theta^{5}=-\frac{1}{2}\left(H \wedge \iota_{v} H\right)=-\frac{1}{2}\left(F \wedge *_{5} F\right) \wedge \theta^{5} \quad$ if $\quad H=* H \quad$ and $\quad \mathcal{L}_{v_{5}} B^{\text {hor }}=0$. 
6d self-dual field as (abelian) 5d Yang-Mills with KK-modes. Due to proposition 2.8, one may regard the general bosonic Perry-Schwarz Lagrangian (definition 2.5) for the self-dual field on $\Sigma^{6}$ compactified on $S^{1}$ as that of (abelian) 5d Yang-Mills theory with a tower of KK-modes included, which is a perspective on the M5-brane theory later advanced in $[35,76]$ (see $[77,3.4 .3])$ ). Here for the abelian bosonic sector, this perspective may be fully brought out by introducing the following notation:

$$
F:=\iota_{v_{5}} H-\mathcal{L}_{v_{5}} B^{\text {hor }} \quad \text { and } \quad \widetilde{F}:=H-F \wedge \theta^{5},
$$

where on the left we have (2.18), as before, while on the right we are introducing notation for the remaining summand in $H$. With this notation, the bosonic PS Lagrangian, assuming self-duality (2.2) as in (2.24), finds the following suggestive expression:

$$
\mathbf{L}^{\mathrm{PS}}=-\frac{1}{2} F \wedge \widetilde{F} \quad \text { if } H=* H,
$$

so that KK-reduction to 5d YM theory, as in proposition 2.8, is now given syntactically simply by replacing $\widetilde{F} \mapsto *_{5} F$. We find the form (2.30) of the PS Lagrangian to be reflected by its super-exceptionalization in proposition 5.1 below; see definition 5.2 and remark 5.8 .

The topological 5d Yang-Mills Lagangian. After dimensional reduction [1, section 6], [2, section 6 \& appendix A], the Perry-Schwarz Lagrangian for the M5-brane is accompanied by a multiple of the topological Yang-Mills Lagrangian $[8,14]$

$$
\mathbf{L}^{\mathrm{tYM}}:=F \wedge F
$$

We find this arise from the super-exceptional embedding construction below in theorem 7.3, see remark 7.5.

\section{Super-exceptional M-geometry}

We recall (in definition 3.3 and proposition 3.5) the "hidden supergroup of $D=11$ supergravity" $[4,10,32]$ interpreted as super-exceptional M-theory spacetime [45, 4.6], [103] and explain, in remark 3.9 , how the results of $[47,48]$ identify this as the correct local target for M5-brane sigma-models.

For definiteness, we make explicit our spinor and Clifford algebra convention:

Definition 3.1 (e.g. [69, proposition A.3 (v)]). We write 32 for the irreducible real representation of $\operatorname{Pin}^{+}(10,1)$, via a Clifford-algebra representation $\left\{\boldsymbol{\Gamma}_{\boldsymbol{a}}\right\}_{a=0}^{10}$ whose generators satisfy the following relations:

$$
\begin{aligned}
\boldsymbol{\Gamma}_{a} \boldsymbol{\Gamma}_{b}+ & \boldsymbol{\Gamma}_{b} \boldsymbol{\Gamma}_{a}=+2 \eta_{a b}:=2 \operatorname{diag}(-1,+1,+1, \cdots,+1)_{a b}, \\
\left(\boldsymbol{\Gamma}_{0}\right)^{2}=-1, & \left(\boldsymbol{\Gamma}_{a}\right)^{2}=+1, \\
\left(\boldsymbol{\Gamma}_{0}\right)^{\dagger}=-\boldsymbol{\Gamma}_{0}, & \left(\boldsymbol{\Gamma}_{a}\right)^{\dagger}=+\boldsymbol{\Gamma}_{a}, \quad \text { for } a \in\{1, \cdots, 10\} .
\end{aligned}
$$


Remark 3.2 (Gamma matrix conventions). definition 3.1 relates to an alternative Clifford algebra convention $\left\{\Gamma_{a}\right\}_{a=0}^{10}$ used in much of the relevant literature (e.g. $\left.[10,32]\right)$ via the relation

$$
\Gamma_{a}:=i \Gamma_{a}
$$

understood inside a complex Dirac representation (see [69, proposition A.3]). We will use " $\Gamma_{a}$ " for exhibiting expressions manifestly compatible with the literature, and " $\boldsymbol{\Gamma}_{a}$ " for emphasizing the actual real $\mathrm{Pin}^{+}(10,1)$-action.

The following, definition 3.3 and proposition 3.5, are a formulation in rational superhomotopy theory due to [45, section 4.5], of the classical supergravity results in [32, section 6], [10, section 3] (see also [4]). The definition 3.3 of super-exceptional spacetime involves a parameter $s$, eq. (3.2), which arises mathematically in proposition 3.5 from different possibilities of decomposing the $H_{3}$-flux on super-exceptional spacetime [10]. We discover the physical meaning of this parameter below in section 5 .

Definition 3.3 (Super-exceptional M-theory spacetime). For parameter

$$
s \in \mathbb{R} \backslash\{0\}
$$

the $D=11, \mathcal{N}=1, n=11$ super-exceptional $M$-theory spacetime $\left(\mathbb{T}^{10,1 \mid \mathbf{3 2}}\right)_{\mathrm{ex}_{s}}$ over ordinary $D=11, \mathcal{N}=1$ super Minkowski spacetime $\mathbb{T}^{10,1 \mid \mathbf{3 2}}$ is the rational super space given dually by the following super dgc-algebra:

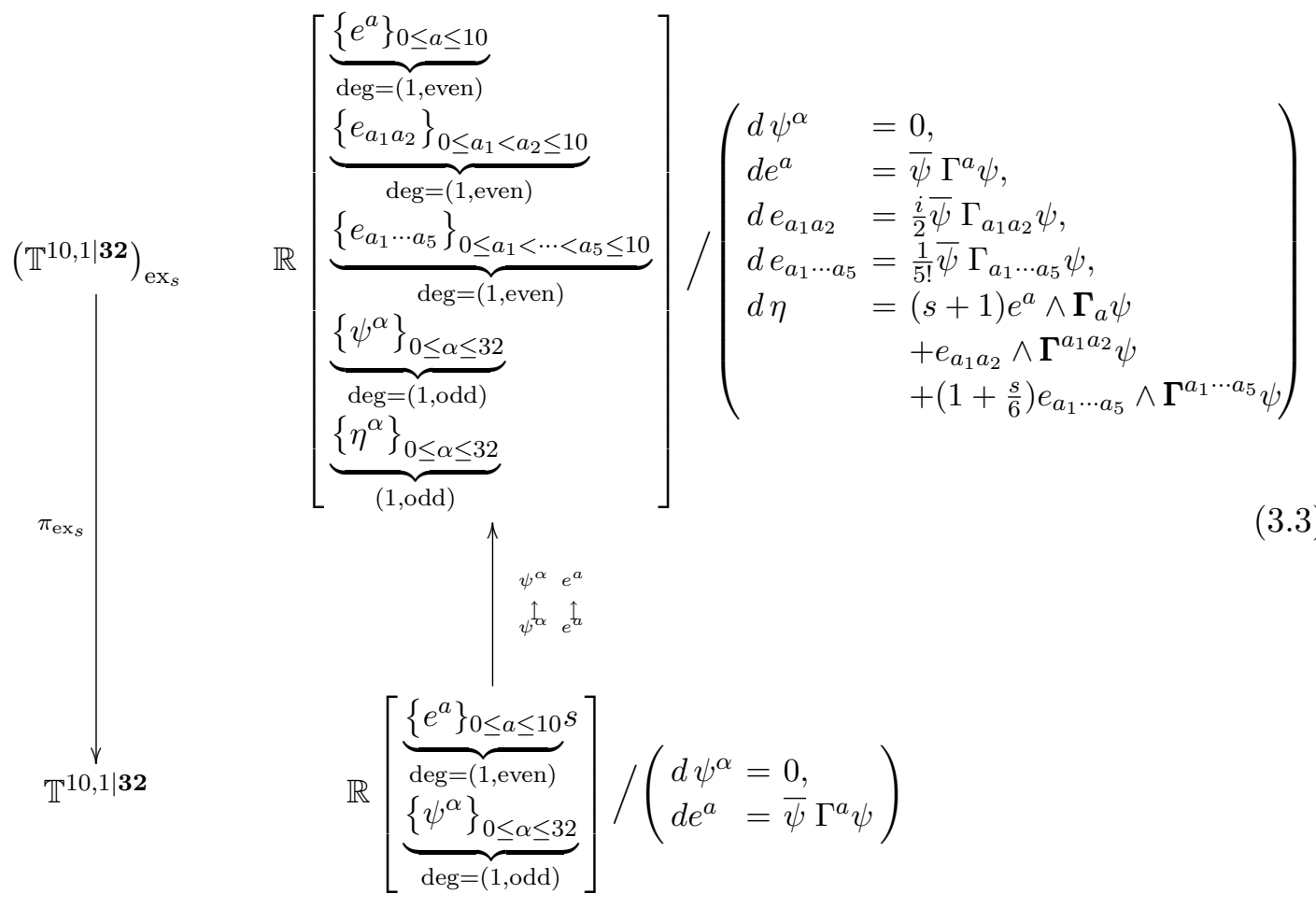

Here the index $\alpha$ ranges over a linear basis of the real $\operatorname{Pin}^{+}(10,1)$-representation 32 and the Clifford generators $\Gamma_{a}$ acting on these are as in definition 3.1; and we use Einstein summation convention with the $e_{a_{1} a_{2}}$ and $e_{a_{1} \cdots a_{5}}$ understood as completely antisymmetrized in their indices. 
Remark 3.4 (Super-exceptional M-theory spacetime as a supermanifold). We may alternatively regard $\left(\mathbb{T}^{10,1 \mid \mathbf{3 2}}\right)_{\mathrm{ex}_{s}}$ from definition 3.3 as a super-manifold with canonical global coordinate functions

$$
C^{\infty}\left(\left(\mathbb{R}^{10,1 \mid \mathbf{3 2}}\right)_{\mathrm{ex}_{s}}\right)=\langle\underbrace{\left(x^{a}\right)}_{\operatorname{deg}=(0, \text { even })}, \underbrace{\left(B_{a_{1} a_{2}}\right)}_{\operatorname{deg}=(0, \text { even })}, \underbrace{\left(B_{a_{1} \cdots a_{5}}\right)}_{\operatorname{deg}=(0, \text { even })}, \underbrace{\left(\theta^{\alpha}\right)}_{\operatorname{deg}=(0, \text { odd })}, \underbrace{\left(\rho^{\alpha}\right)}_{\operatorname{deg}=(0, \text { odd })}\rangle,
$$

hence with bosonic part being the exceptional tangent bundle (1.1) for maximal $n=11$ :

$$
\left(\left(\mathbb{R}^{10,1 \mid \mathbf{3 2}}\right)_{\mathrm{ex}_{s}}\right)_{\mathrm{bos}} \simeq T \mathbb{R}^{10,1} \oplus \wedge^{2} T^{*} \mathbb{R}^{10,1} \oplus \wedge^{5} T^{*} \mathbb{R}^{10,1},
$$

and with super-group structure such that the Chevalley-Eilenberg algebra in definition 3.3 identifies with the super de Rham dgc-algebra of left-invariant (hence translationally supersymmetric) super-differential forms:

$$
\begin{aligned}
\mathrm{CE}\left(\left(\mathbb{T}^{10,1 \mid \mathbf{3 2}}\right)_{\mathrm{ex}_{s}}\right) \stackrel{\simeq}{\longrightarrow} \Omega_{\mathrm{li}}^{\bullet}\left(\left(\mathbb{T}^{10,1 \mid 32}\right)_{\mathrm{ex}_{s}}\right) \hookrightarrow & \longrightarrow\left(\left(\mathbb{R}^{10,1 \mid \mathbf{3 2}}\right)_{\mathrm{ex}_{s}}\right) . \\
\psi^{\alpha} \longmapsto & d \theta^{\alpha} \\
\eta^{\alpha} \longmapsto & d \rho^{\alpha} \\
e^{a} \longmapsto & d x^{a}+\bar{\theta} \Gamma^{a} d \theta \\
e_{a_{1} a_{2}} \longmapsto & d\left(B_{a_{1} a_{2}}\right)+\frac{i}{2} \bar{\theta} \Gamma_{a_{1} a_{2}} d \theta \\
e_{a_{1} \cdots a_{5}} \longmapsto & \frac{1}{\alpha_{0}(s)} d\left(B_{a_{1} \cdots a_{5}}\right)+\frac{1}{5 !} \bar{\theta} \Gamma_{a_{1} \cdots a_{5}} d \theta .
\end{aligned}
$$

Beware the bracketing in the last two lines on the right, in contrast to $(d B)_{a_{1} \cdots a_{3}}$ etc. The bosonic component of the generator $e_{a_{1} a_{2}}$ is the de Rham differential of the bosonic component functions of a 2 -form $B:=B_{a_{1} a_{2}} d x^{a} \wedge d x^{b}$

$$
d\left(B_{a_{1} a_{2}}\right)=d x^{\mu} \partial_{\mu} B_{a_{1} a_{2}}
$$

(without antisymmetryization over all three indices, at this point), instead of the component functions of the de Rham differential of a 2 -form, which is instead obtained by anti-symmetrizing over all three indices

$$
H:=\left(d\left(B_{a_{1} a_{2}}\right)\right) \wedge d e^{a_{1}} \wedge d e^{a_{2}}
$$

as in (3.10) below. This has a crucial effect in the following discussion; see lemma 5.1.

Proposition 3.5 (Transgression of M2-cocycle on super-exceptional spacetime). For $s \in$ $\mathbb{R} \backslash\{0\}$, the fermionic extension of exceptional tangent superspacetime $\mathbb{R}_{\mathrm{ex}_{s}}^{10,1 \mid \mathbf{3 2}}$ ((3.3)), regarded as fibered over $11 d$ super-Minkowski spacetime $\mathbb{T}^{10,1 \mid \mathbf{3 2}}$ carries a transgression element for the M2-brane 4-cocycle $\mu_{\mathrm{M} 2}(1.2)$ :

$$
H_{\mathrm{ex}_{s}} \in \mathrm{CE}\left(\left(\mathbb{T}^{10,1 \mid 32}\right)_{\mathrm{ex}_{s}}\right) \quad \text { such that } d H_{\mathrm{ex}_{s}}=\left(\pi_{\mathrm{ex}_{s}}\right)^{*} \mu_{\mathrm{M} 2}
$$

given by

$$
H_{\mathrm{ex}_{s}}=\underbrace{\alpha_{0}(s) e_{a_{1} a_{2}} \wedge e^{a_{1}} \wedge e^{a_{2}}}_{=\left(d B_{a_{1} a_{2}}\right) \wedge e_{a_{2}} \wedge e_{a_{3}}=: H}-\alpha_{3}(s) \epsilon_{a_{1} \cdots a_{5} b_{1} \cdots b_{5} c} e^{a_{1} \cdots a_{5}} \wedge e^{b_{1} \cdots b_{5}} \wedge e^{c}+\mathrm{H}_{s}^{\mathrm{fib}}
$$


with

$$
\begin{aligned}
\left(H_{s}^{\mathrm{fib}}\right)_{\mathrm{bos}}= & \alpha_{1}(s) e^{a_{1}} a_{2} \wedge e_{a_{3}}^{a_{2}} \wedge e^{a_{3}} a_{1} \\
& +\alpha_{2}(s) e_{b_{1} a_{1} \cdots a_{4}} \wedge e^{b_{1}} b_{2} \wedge e^{b_{2} a_{1} \cdots a_{4}} \\
& +\alpha_{4}(s) \epsilon_{\alpha_{1} \cdots \alpha_{6} b_{1} \cdots b_{5}} e^{a_{1} a_{2} a_{3}} c_{c_{1} c_{2}} \wedge e^{a_{4} a_{5} a_{6} c_{1} c_{2}} \wedge e^{b_{1} \cdots b_{5}}
\end{aligned}
$$

and

$$
\left(H_{s}^{\mathrm{fib}}\right)_{\mathrm{ferm}}=-\frac{1}{2} \bar{\eta}_{\alpha} \wedge \psi^{\beta} \wedge\left(\beta_{1}(s)\left(\Gamma_{a}\right)^{\alpha}{ }_{\beta} e^{a}+\beta_{2}(s)\left(\Gamma^{a_{1} a_{2}}\right)^{\alpha}{ }_{\beta} e_{a_{1} a_{2}}+\beta_{3}(s)\left(\Gamma^{a_{1} \cdots a_{5}}\right)^{\alpha}{ }_{\beta} e_{a_{1} \cdots a_{5}}\right),
$$

for analytic functions $\alpha_{i}, \beta_{j}$ of the parameter $s \in \mathbb{R} \backslash\{0\}$ with the following zeros

$$
\begin{aligned}
& \alpha_{0}(s) \neq 0 \\
& \alpha_{1}(s)=0 \Leftrightarrow s=-3 \quad \beta_{1}(s)=0 \Leftrightarrow s=-3 / 2 \\
& \alpha_{2}(s)=0 \Leftrightarrow s=-6 \quad \beta_{2}(s)=0 \Leftrightarrow s=-3 \\
& \alpha_{3}(s)=0 \Leftrightarrow s=-6 \quad \beta_{3}(s)=0 \Leftrightarrow s=-6 . \\
& \alpha_{4}(s)=0 \Leftrightarrow s=-6
\end{aligned}
$$

Example 3.6 (Special parameter-value for super-exceptional geometry). If the parameter $s(3.2)$ in definition 3.3 and proposition 3.5 takes the value

$$
s=-3
$$

the transgression element in proposition 3.5 has the property, from (3.9), that up to terms proportional to the 5 -index tensor $e_{\alpha_{1} \cdots \alpha_{5}}$, its only dependence on $d B_{a_{1} a_{2}}:=\alpha_{0}(s) e_{a_{1} a_{2}}$ is through the leading term $H:=\left(d B_{a_{2} a_{3}}\right) \wedge d x^{a_{2}} \wedge d x^{a_{3}}$; concretely:

$$
H_{\operatorname{ex}_{(s=-3)}}=\underbrace{\left(d B_{a_{2} a_{3}}\right) \wedge d x^{a_{2}} \wedge d x^{a_{3}}}_{H}-\frac{1}{2} \beta_{1}(-3) \cdot \bar{\eta} \Gamma_{a} \psi \wedge e^{a}+\mathcal{O}\left(\left\{e_{a_{1} \cdots a_{5}}\right\}\right),
$$

where the last term $\mathcal{O}\left(\left\{e_{a_{1} \cdots a_{5}}\right\}\right)$ denotes summands that vanish when the 5 -index generators $e_{a_{1} \cdots a_{5}}$ are set to zero.

We will see below in proposition 5.1 (ii). that in super-exceptional M-geometry at value $s=-3$ the bosonic Perry-Schwarz Lagrangian appears naturally.

The point of the super-exceptional M-theory spacetime from definition 3.3 is that it is a super-manifold (via remark 3.4) which approximates the universal super 3-stack $\widehat{\mathbb{T}^{10,1 \mid 32}}$ classified by the super M2-brane cocycle (1.2). We record this phenomenon (which can be traced back to the "hidden supergroup of $11 \mathrm{~d}$ supergravity" in [32]):

Lemma 3.7 (Super-exceptional M-spacetime from M2-brane super-2-gerbe [45, 4.6]). We have a commuting diagram of the form

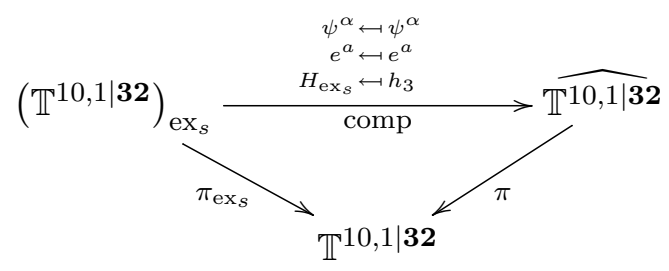

mapping the super-exceptional spacetime from definition 3.3 to the homotopy fiber of the M2-brane cocycle from (1.2), such that the defining degree-3 generator $h_{3}$ on the right is pulled back to the transgression element $H_{\mathrm{ex}_{s}}$ from proposition 3.5. 
Hence we say:

Definition 3.8 (Super-exceptional M5-brane cocycle). The super-exceptional M5-brane cocycle, to be denoted $\mathbf{d L}_{\text {ex }}^{\mathrm{WZ}}$, is the pullback of the super M5-brane cocycle (1.2) to the superexceptional M-theory spacetime (definition 3.3) along the decomposition morphism (3.11):

$$
\begin{aligned}
\mathbf{d L}_{\mathrm{ex}_{s}}^{\mathrm{WZ}} & :=\operatorname{comp}^{*}(\frac{1}{2} h_{3} \wedge \overbrace{\pi^{*} \mu_{\mathrm{M} 2}}^{=d h_{3}}+\pi^{*} \mu_{\mathrm{M} 5}) \\
& =\frac{1}{2} H_{\mathrm{ex}_{s}} \wedge\left(\pi_{\mathrm{ex}_{s}}\right)^{*} \mu_{\mathrm{M} 2}+\left(\pi_{\mathrm{ex}_{s}}\right)^{*} \mu_{\mathrm{M} 5} \\
& =\frac{1}{2} H_{\mathrm{ex}_{s}} \wedge d H_{\mathrm{ex}_{s}}+\frac{1}{5 !}\left(\bar{\psi} \Gamma_{a_{1} \cdots a_{5}} \psi\right) \wedge e^{a_{1}} \wedge \cdots e^{a_{5}} \in \mathrm{CE}\left(\left(\mathbb{R}^{10,1 \mid 32}\right)_{\mathrm{ex}_{s}}\right) .
\end{aligned}
$$

We close by commenting on the role and meaning of these constructions:

Remark 3.9 (Super-exceptional spacetime as target for M5-brane sigma-models).

(i) The super-exceptional spacetime (definition 3.3), when regarded as a super-group of super-translations along itself, is what [32, section 6] called the "hidden supergroup of 11-dimensional supergravity"; motivated there by the purely algebraic desideratum of finding a super Lie 1-algebra over which the would-be M2-brane cocycle trivializes (proposition 3.5), as opposed to the super Lie 3-algebra $\mathfrak{m} 2 \mathfrak{b r a n e}$ [39] on which it does so universally.

(ii) However, the actual meaning or role of this "hidden supergroup" had remained open; as it was not used for the re-derivation of $D=11$ supergravity in [32], but discussed as an afterthought. In particular, the meaning or role of the extra fermion field required by super-exceptional spacetime had remained open [4, p. 3] and the relation of the bosonic fields to exceptional M-geometry had remained unnoticed except in [114] and then more recently in $[9$, p. 6$],[45,103]$.

(iii) But in [47, proposition 4.31], [48, proposition 4.4], we found that the M5-brane sigma model in a given C-field background is characterized as making the outermost square of the following diagram homotopy-commute, here now displayed for flat super-spacetimes instead of curved topological spacetimes:

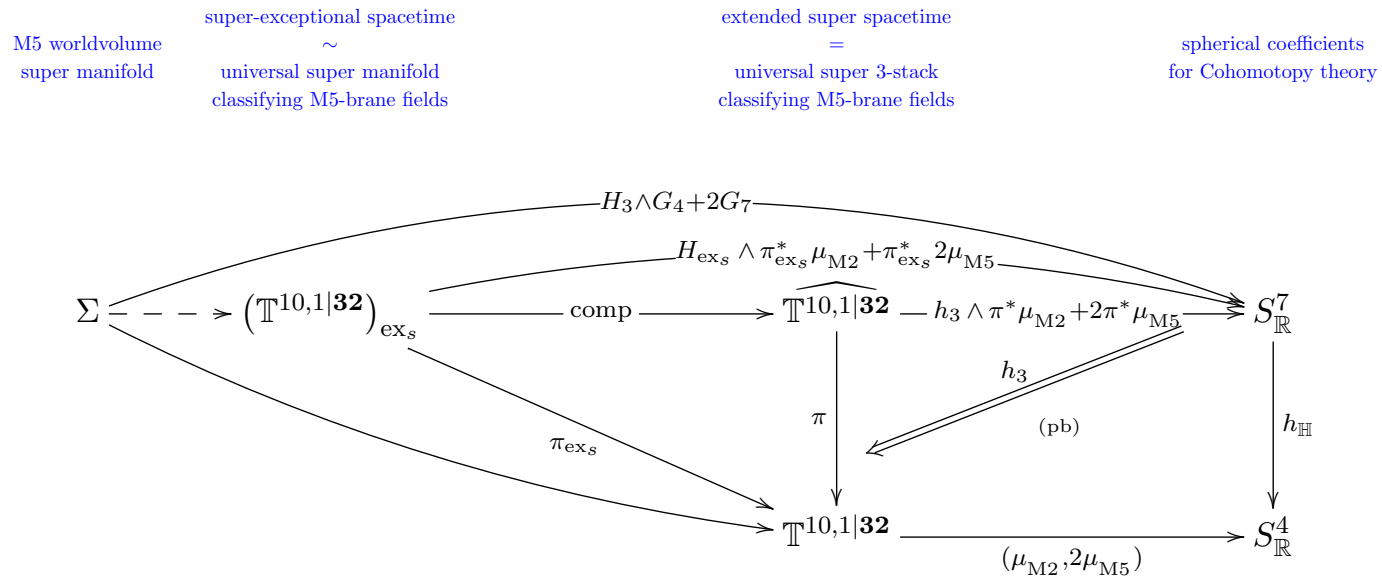


Any such outer square factors universally through the homotopy pullback square shown on the right, which exhibits the M2-brane extended super spacetime $\widehat{\mathbb{T}^{10,1 \mid 32}}$ (1.2) as the super moduli 3-stack classifying M5-brane sigma-model fields [42]. But now with the (extended) worldvolume $\Sigma$ itself a super manifold, this factorization through $\widehat{\mathbb{T}^{10,1 \mid 32}}$ is to be further factorized, as shown by the dashed map, through an actual super-manifold still classifying these fields. This is the role of the superexceptional spacetime $\left(\mathbb{R}^{10,1 \mid \mathbf{3 2}}\right)_{\mathrm{ex}_{s}}[45$, section 4.6$]$ : it is the actual super manifold which serves as a stand-in for the classifying super space $\widehat{\mathbb{T}^{10,1 \mid 32}}$ (which is not itself a super-manifold).

(iv) The key consequence of $\left(\mathbb{T}^{10,1 \mid 32}\right)_{\operatorname{ex}_{s}}$ being a super-manifold, is that the indecomposable degree-3 generator $h_{3}$ on $\widehat{\mathbb{T}^{10,1 \mid 32}}(1.2)$, which has trivial contraction with any vector field, pulls back to the decomposable 3 -form $H_{\mathrm{ex}_{s}}$ on $\left(\mathbb{T}^{10,1 \mid \mathbf{3 2}}\right)_{\mathrm{ex}_{s}}$ (by lemma 3.7), which, like any differential form on a super manifold, in general has non-trivial contraction with vector fields. Below in section 5 it is such a non-trivial contraction of $H_{\mathrm{ex}_{s}}$ with a vector field on the super-exceptional spacetime, which makes the Perry-Schwarz Lagrangian appear. The analogous contraction with $h_{3}$ on $\widehat{\mathbb{T}^{10,1 \mid 32}}$ would vanish, which is the reason why the embedding construction of the M5-brane does not work with extended super-spacetime, but requires passing to super-exceptional spacetime.

Lemma 3.10 ([45, proposition 4.26]). The canonical $\operatorname{Pin}^{+}(10,1)$ action on superspacetime $\mathbb{R}^{10,1 \mid \mathbf{3 2}}$ lifts to super-exceptional spacetime $\left(\mathbb{R}^{10,1 \mid \mathbf{3 2}}\right)_{\mathrm{ex}_{s}}$ (definition 3.3), such that $\operatorname{Spin}(10,1) \hookrightarrow \operatorname{Pin}^{+}(10,1)$ acts in the evident way, while reflection $\rho$ along the $a_{r}$-axis acts dually as follows: ${ }^{1}$

$$
\rho^{*}:\left\{\begin{aligned}
e^{a} & \longmapsto\left\{\begin{aligned}
-e^{a} \mid a=a_{r} \\
e^{a} \mid \text { otherwise }
\end{aligned}\right. \\
e_{a_{1} a_{2}} & \longmapsto\left\{\begin{aligned}
-e_{a_{1} a_{2}} \mid a_{1}, a_{2} \neq a_{r} \\
e_{a_{1} a_{2}} \mid \text { otherwise }
\end{aligned}\right. \\
e_{a_{1} \cdots a_{5}} & \longmapsto\left\{\begin{array}{c}
-e_{a_{1} \cdots a_{5}} \mid \text { one of the } a_{i}=a_{r} \\
e_{a_{1} \cdots a_{5}} \mid \text { otherwise }
\end{array}\right. \\
\psi & \Gamma_{a_{r}} \psi \\
\eta & -\Gamma_{a_{r}} \eta .
\end{aligned}\right.
$$

\section{Super-exceptional MK6- and $\frac{1}{2}$ M5-geometry}

We now consider the exceptionalization (definition 4.6 below) of the local super-geometry of M-theory compactified on $\mathbb{H}_{/ / G_{\mathrm{ADE}}} \times S_{/ / \mathbb{Z}_{2}}^{1}[21,74]$, hence of Hořava-Witten heterotic

\footnotetext{
${ }^{1}$ Beware that this is saying that the generator $e_{a_{1} a_{2}}$ picks up a sign precisely if its indices do not take the value $a_{r}$.
} 
M-theory on an ADE-orbifold, or, equivalently, of non-perturbative type I' string theory with D6-branes intersecting the O8-plane in a $\frac{1}{2}$ NS5-brane $[51,6][5$, p. 18]. Therefore, following [69, example 2.2.7], we will speak here of the " $\frac{1}{2} \mathrm{M} 5$ super-spacetime", for emphasis of the full 11-dimensional perspective; details are given in remark 4.2.

It is traditionally understood that this compactification is one of two possible ways of obtaining classes of $D=6, \mathcal{N}=(1,0)$ superconformal field theories from M-theory [33, section 6]. We make this mathematically concrete with proposition 4.4 below, whose proof shows that the particular spinor structure of the $\frac{1}{2} \mathrm{M} 5$-locus (definition 4.1 below) is what allows a lift of the spacetime isometry along the M-theory circle to a symmetry also of the exceptionalized super-spacetime, including the exceptionalized 3-flux density on the M5-brane.

We use this to identify in proposition 5.1 the exceptional pre-image of the bosonic 2-flux density F. Finally, we show in proposition 5.3 that this induces the super 2-flux of super Yang-Mills theory thereby, in particular, identifying the extra fermion field on exceptional super-spacetime with the M-theoretic avatar of the gaugino field in 10d heterotic string theory.

Definition 4.1 (Spinor projection of $\frac{1}{2} \mathrm{M} 5$-locus). For 32 the Pin-representation from definition 3.1 , let $P_{\mathbf{8}}$ denote the linear projection

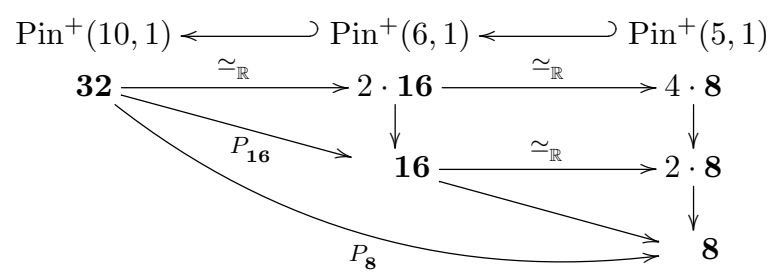

onto the joint fixed locus of $\boldsymbol{\Gamma}_{5}$ and $\boldsymbol{\Gamma}_{6789}$. Hence this is characterized by the following

$$
\begin{array}{rll}
\operatorname{MK} 6 & \Gamma_{6789}\left(P_{\mathbf{1 6}} \psi\right)=\left(P_{\mathbf{1 6}} \psi\right) & \mathcal{O} 6 \\
\frac{1}{2} \mathrm{M} 5 & \Gamma_{6789}\left(P_{\mathbf{8}} \psi\right)=\left(P_{\mathbf{8}} \psi\right) & \mathcal{I} 5 \\
& \Gamma_{5}\left(P_{\mathbf{8}} \psi\right)=\left(P_{\mathbf{8}} \psi\right) \\
& \Gamma_{56789}\left(P_{\mathbf{8}} \psi\right)=\left(P_{\mathbf{8}} \psi\right)
\end{array}
$$

Remark 4.2 (The $\frac{1}{2} \mathrm{M} 5$-locus). The spinor projections in definition 4.1 correspond to the $\frac{1}{2}$ M5 = MK6 $\cap$ MO9 super spacetime [51, 6], [33, 6], [5, p. 18 ]; see [69, example 2.2.7] in the present context:

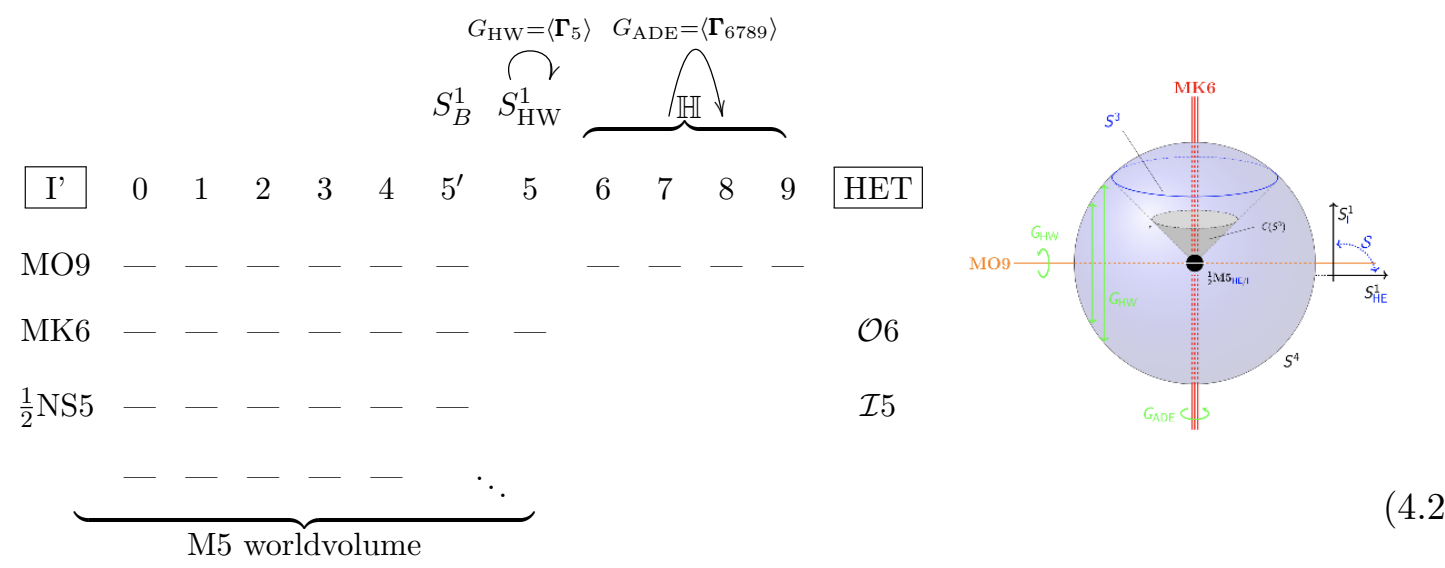


1. Black branes. The appropriate names of the fixed loci depend on the duality frame: if the circle $S_{A}^{1}$ inside the quaternion space $\mathbb{H}$ on which $G_{\mathrm{A}}$ acts is taken for M/IIA duality, then the result is type I' and the fixed loci are named as shown on the left (see also e.g. [56, around figure 2], [59]). On the other hand, if the circle $S_{\mathrm{HW}}^{1}$ on which $G_{\mathrm{HW}}$ acts is taken for M/IIA duality, the result is HET on a $G_{\mathrm{ADE}}$-orbifold, and labels as used in [51, p. 8] are as shown on the right. Moreover, under T-duality along $S_{B}^{1}$ the I'-perspective turns into a configuration of a IIB NS5-brane parallel to an O9-plane (see, e.g., [57]).

2. Fundamental (sigma-model) brane. The last line in the above diagram indicates the extension of the actual M5-brane worldvolume for which we are to construct a Lagrangian density. This is not required to be fixed by $\boldsymbol{\Gamma}_{5}$ and hence may stretch along the 5'-5-plane/torus "at an angle", partially wrapping around the $S_{\mathrm{HW}}^{1}$, partially running parallel to it [118, p. 9], corresponding to D4/NS5 bound states in IIA [118, section 2] and $(p, q)$-5brane webs in IIB [3], or rather their further intersection with an O-plane [57] (see [59, around figure 10]).

The following definition generalizes the super MK6-spacetime regarded as a $\mathbb{Z}_{2}$-fixed locus inside $D=11, \mathcal{N}=1$ super Minkowski spacetime [45, proposition 4.7, example 2.2.5] to a $\mathbb{Z}_{2}$-fixed locus inside the super-exceptional M-theory spacetime:

Definition 4.3 (Super-exceptional MK6-spacetime). For $s \in \mathbb{R} \backslash\{0\}$, we say that superexceptional MK6-spacetime $\left(\mathbb{R}^{6,1 \mid \mathbf{1 6}}\right)_{\mathrm{ex}_{s}}$ is the fixed locus inside the super-exceptional Mtheory spacetime (definition 3.3) of the $\mathbb{Z}_{2} \hookrightarrow \operatorname{Pin}^{+}(10,1)$-action of lemma 3.10 generated from the element $\boldsymbol{\Gamma}_{6789} \in \operatorname{Pin}^{+}(10,1)$ as in proposition 4.1. Hence, from the super dgcalgebra (3.3) and lemma 3.10, this is the rational super space given dually by the following super dgc-algebra:

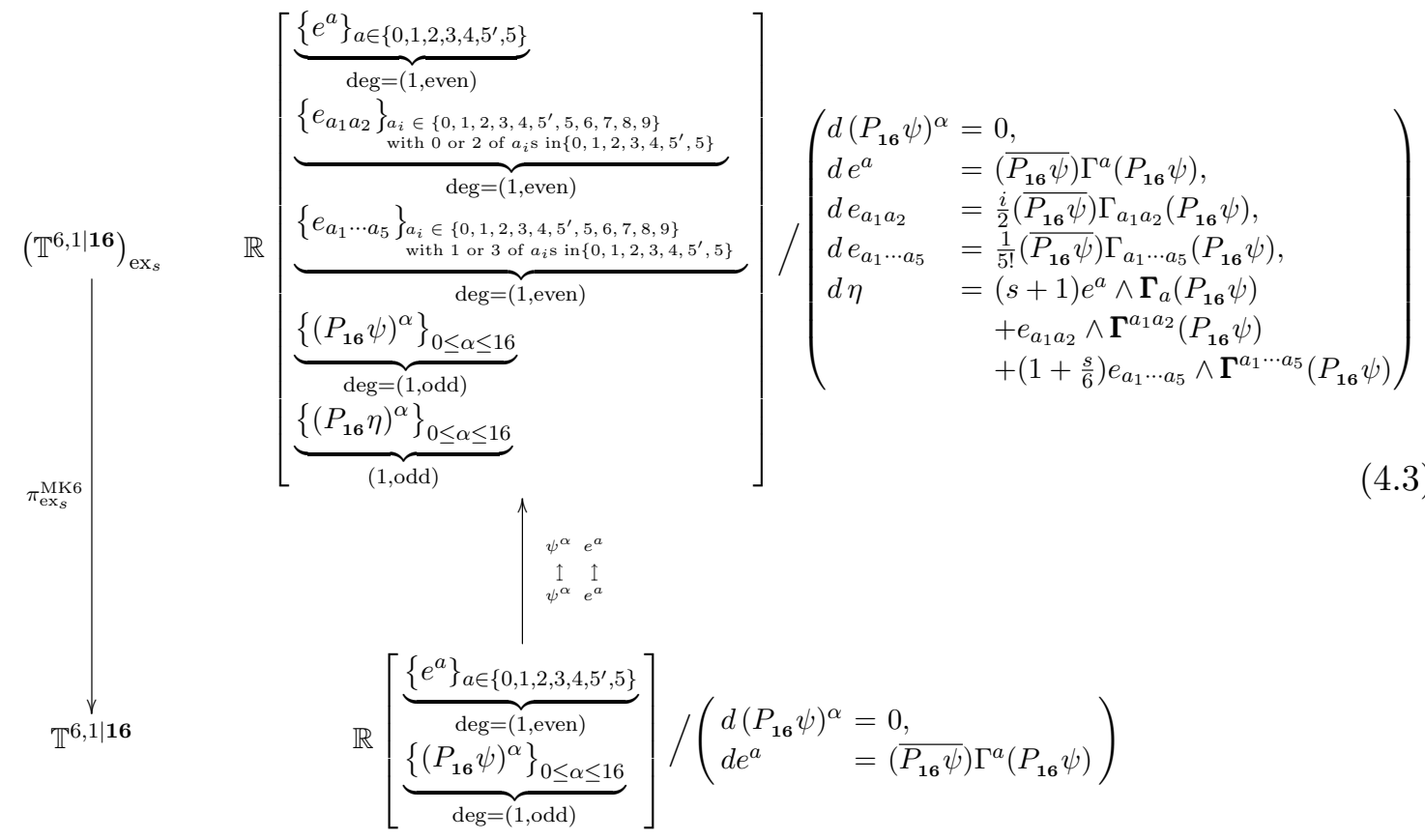

We now incorporate spacetime isometries. 
Proposition 4.4 (Lift of isometries to super-exceptional MK6-spacetime). For all parameters $s$ from (3.2), distinct from -6, i.e., for

$$
s \in \mathbb{R} \backslash\{0,-6\},
$$

and for all $a \in\left\{1,2,3,4,5^{\prime}, 5\right\}$, the infinitesimal superspace symmetry $v_{a}$

$$
v_{a} \in \Gamma\left(T \mathbb{R}^{6,1 \mid \mathbf{1 6}}\right), \quad \iota_{v_{a}}:\left\{\begin{array}{rl}
e^{b} & \mapsto \delta_{b}^{a} \\
\left(P_{\mathbf{1 6}} \psi\right)^{\alpha} & \mapsto 0
\end{array}, \quad \mathcal{L}_{v_{a}}:=\left[d, \iota_{v_{a}}\right]=0\right.
$$

of the super MK6-spacetime lifts to the super-exceptional MK6-spacetime (definition 4.3) as

$$
v_{a}^{\mathrm{ex}_{s}}:=v_{a}-\frac{1+s}{1+\frac{s}{6}} \frac{1}{5 !} v^{a 6789}+\chi^{\alpha} v_{\alpha}^{\eta} \in \Gamma\left(T \mathbb{R}^{5,1 \mid 8}\right), \quad \iota_{v_{a}^{\mathrm{ex}} \mathrm{s}}:\left\{\begin{aligned}
e^{b} & \mapsto \delta_{b}^{a} \\
e_{a_{1} a_{2}} & \mapsto 0 \\
e_{a_{1} \cdots a_{5}} & \mapsto-\frac{1+s}{1+\frac{s}{6}} \epsilon_{a 6789}^{a_{1} \cdots a_{5}} \\
(P \psi)^{\alpha} & \mapsto 0 \\
(P \eta)^{\alpha} & \mapsto(P \chi)^{\alpha}
\end{aligned}\right.
$$

for any odd-graded constants

$$
\underbrace{(P \chi)^{\alpha}}_{\operatorname{deg}=(0, \text { odd })}
$$

in that

$$
\mathcal{L}_{v_{a}^{\text {ex }_{s}}}:=\left[d_{\mathrm{ex}_{s}}, \iota_{v_{a}^{\mathrm{ex}_{s}}}\right]=0 \quad \text { on }\left(\mathbb{R}^{6,1 \mid \mathbf{1 6}}\right)_{\mathrm{ex}_{s}} .
$$

Proof. Direct inspection of the defining differential relations in (4.3) shows that the derivation $\mathcal{L}_{v_{a}^{e_{s}}}$ evidently vanishes on all generators except possibly on $\eta$. The action on $\eta$ is computed as follows:

$$
\begin{aligned}
\mathcal{L}_{v_{a}^{\mathrm{ex}}}\left(P_{\mathbf{1 6}} \eta\right) & =\iota_{v_{a}^{\mathrm{ex}}} d(P \eta)+\underbrace{d \overbrace{\iota_{v_{5}^{\mathrm{ex}_{s}}}(P \eta)}^{=(P \chi)^{\alpha}=\text { const }}}_{=0} \\
& =\iota_{v_{a}^{\text {exs }}}\left((s+1) e^{a_{1}} \boldsymbol{\Gamma}_{a_{1}}(P \psi)+e_{a_{1} a_{2}} \boldsymbol{\Gamma}^{a_{1} a_{2}}(P \psi)+\left(1+\frac{s}{6}\right) e_{a_{1} \cdots a_{5}} \boldsymbol{\Gamma}^{a_{1} \cdots a_{5}}(P \psi)\right) \\
& =(s+1) \boldsymbol{\Gamma}_{a}\left(P_{\mathbf{1 6}} \psi\right)-\left(1+\frac{s}{6}\right) \frac{1+s}{1+\frac{s}{6}} \boldsymbol{\Gamma}_{a} \underbrace{\Gamma^{6789}\left(P_{\mathbf{1 6}} \psi\right)}_{=\left(P_{\mathbf{1 6}} \psi\right)} \\
& =0
\end{aligned}
$$

where under the brace we used the defining property (4.1) of the spinor projections of definition 4.1 .

With the super-exceptional lift of the circle isometry given, we have the corresponding super-exceptional version of the projection (2.9) onto horizontal differential forms.

Definition 4.5 (Super-exceptional horizontal projection). We say that projection onto the super-exceptional horizontal component is the operation

$$
(-)^{\text {horex }_{s}}:=\frac{1}{2}\left(\mathrm{id}-e^{5} \wedge \iota_{v_{5}^{\mathrm{ex}}}\right): \mathrm{CE}\left(\mathbb{T}^{5,1 \mid \mathbf{8}} \times \mathbb{T}^{1}\right)_{\mathrm{ex}_{s}} \longrightarrow \mathrm{CE}\left(\mathbb{T}^{5,1 \mid \mathbf{8}} \times \mathbb{T}^{1}\right)_{\mathrm{ex}_{s}}
$$


on the CE-algebra (FDA) of the super-exceptional $\frac{1}{2}$ M5-spacetime (definition 4.6), where $\iota_{v_{5}}^{\mathrm{ex}_{s}}$ is the contraction (4.6) from proposition 4.4

The next definition 4.6 formalizes the $\frac{1}{2} \mathrm{M} 5$-locus (as in remark 4.2 ) inside the superexceptional MK6-spacetime (as formalized by definition 4.3) without discarding the ambient MK6 spacetime, but breaking its supersymmetry from $D=7, \mathcal{N}=1$, to $D=6$, $\mathcal{N}=(1,0)$. Hence the generators of the MK6 super dgc-algebra ("FDA") are all retained, but all spinors on the right of the differential relations (4.3) get projected not just by $P_{16}$ but by $P_{8}$ (as in definition 4.1):

Definition 4.6 (Super-exceptional $\frac{1}{2} \mathrm{M} 5$ spacetime). For $s \in \mathbb{R} \backslash\{0,-6\}$, we say that the super-exceptional $\frac{1}{2} \mathrm{M} 5$-spacetime is the rational super space given dually by the same super dgc-algebra (4.3) as that of the super-exceptional MK6 of definition 4.3, but with spinor projections $P_{\mathbf{8}}$ instead of just $P_{\mathbf{1 6}}$ (definition 4.1) on the right of the differential relations:

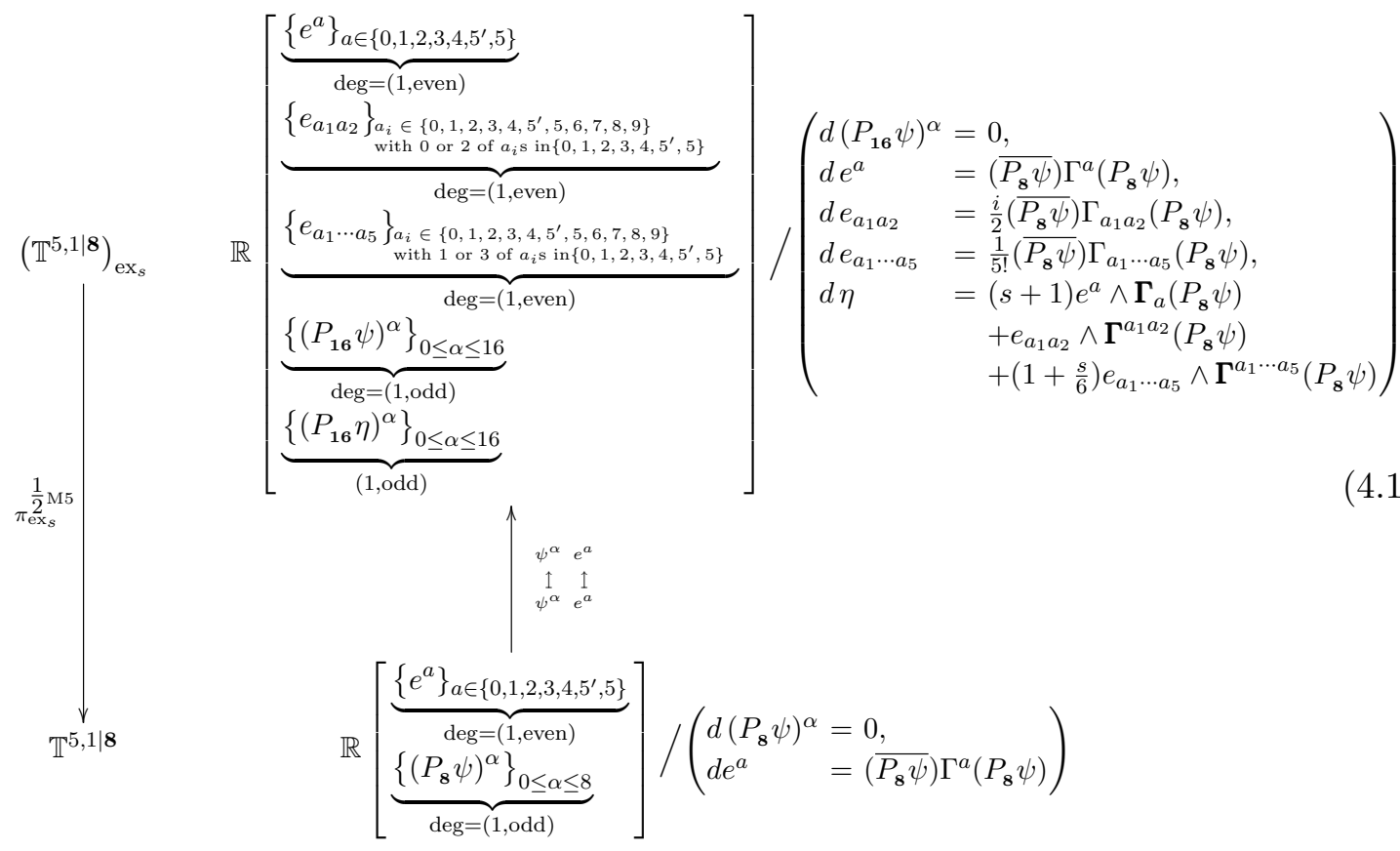

Directly analogous to definition 4.6 we may apply the heterotic spinor projection already on the full super-exceptional M-theory spacetime:

Definition 4.7 (Super-exceptional heterotic M-theory spacetime). For $s \in \mathbb{R} \backslash\{0\}$, we say that the $n=11$ super-exceptional heterotic M-theory spacetime

$$
\begin{aligned}
& \left(P_{16} \psi\right) \hookleftarrow \psi \\
& \begin{array}{lll}
\eta & \leftrightarrow \eta \\
e^{a} & \leftrightarrow e^{a}
\end{array} \\
& e_{a_{1} a_{2}} \longleftrightarrow e_{a_{1} a_{2}} \\
& \left(\mathbb{T}^{9,1 \mid \mathbf{1 6}} \times \mathbb{T}^{1}\right)_{\mathrm{ex}_{s}} \underbrace{e_{a_{1} \cdots a_{5}} \leftrightarrow e_{a_{1} \cdots a_{5}}} \longrightarrow\left(\mathbb{T}^{10,1 \mid \mathbf{3 2}}\right)_{\mathrm{ex}_{s}}
\end{aligned}
$$

is the rational super space given dually by the same super dgc-algebra (3.3) as that of the $n=11$ super-exceptional M-theory spacetime of definition 3.3, but with spinor projections $P_{16}$ (definition 4.1) on the right of the differential relations. 
The following is a direct consequence of the above definitions:

Lemma 4.8 (Super-exceptional embeddings). We have a diagram of consecutive super embeddings covered by super-exceptional embeddings of

(i) the super-exceptional $\frac{1}{2} \mathrm{M} 5$-spacetime (definition 4.6) inside the MK6-spacetime (definition 4.3) inside the $n=11$ super-exceptional $M$-theory spacetime (definition 3.3) as follows:

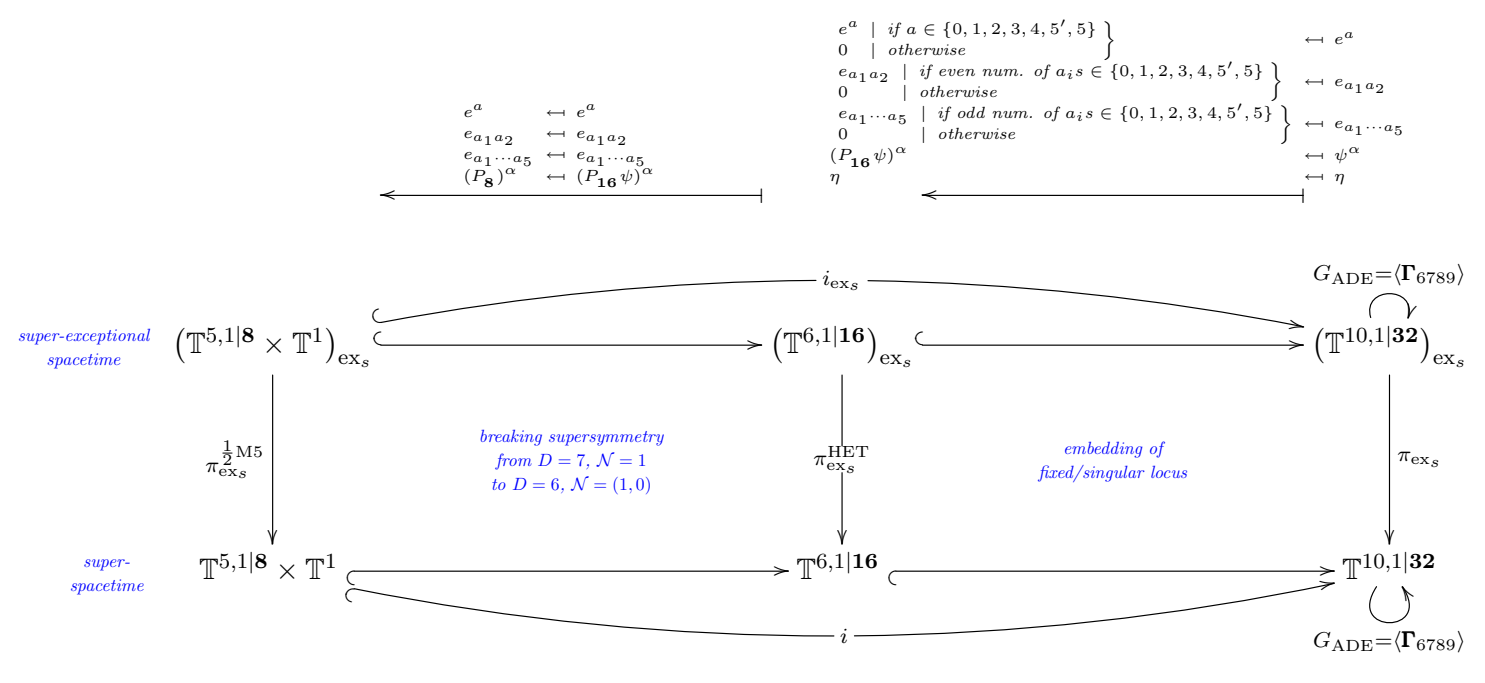

$\frac{1}{2} \mathrm{M} 5$

(ii) the super-exceptional $\frac{1}{2} \mathrm{M} 5$-spacetime (definition 4.6) inside the super-exceptional heterotic $M$-theory spacetime (definition 4.7) inside the $n=11$ super-exceptional $M$ theory spacetime (definition 3.3) as follows:

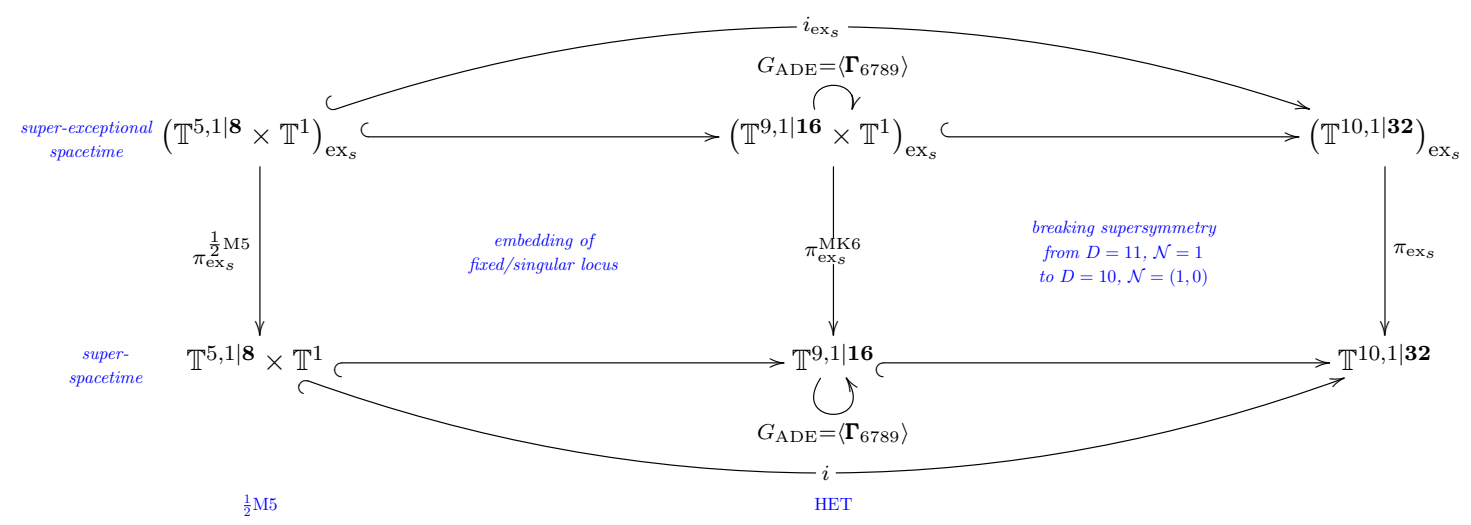

Remark 4.9 (The M5 locus for admissible values of the parameters). Henceforth we declare that the parameter $s$ from (3.2) is distinct from -6, i.e. $s \in \mathbb{R} \backslash\{0,-6\}$ as in (4.4). In this case, proposition 4.4 applies and we have, in summary, the situation as shown in 
the following diagram:

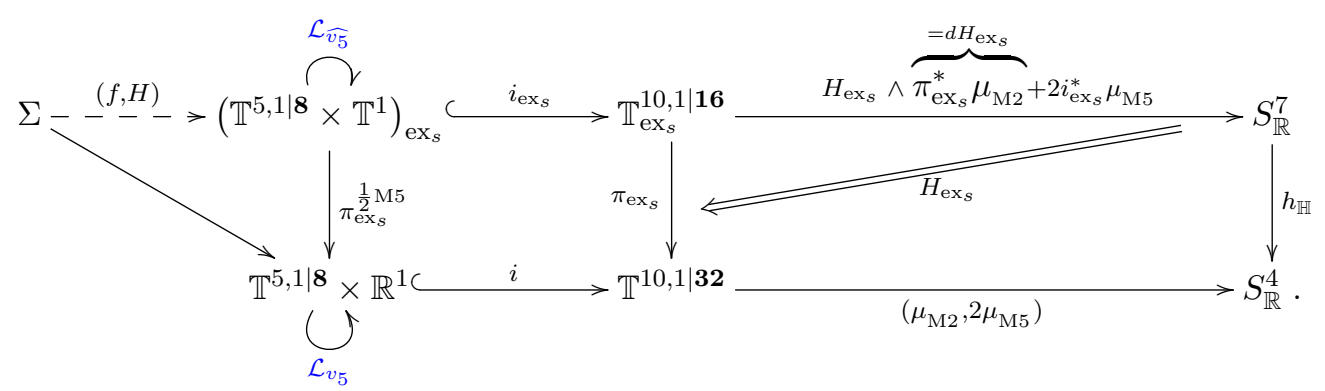

Remark 4.10 (Alternative brane configuration MO1\|MO9). For the case when the parameter value is $s=-6$ after all, there is an alternative brane configuration one may consider, namely the configuration of an "M-wave" (see [69, 2.2.3]) inside an MO9-plane, i.e., with the spinor projection (4.1) replaced by the following:

$$
\begin{array}{ll}
\text { MO9 } & \boldsymbol{\Gamma}_{9}(P \psi)=(P \psi), \\
\text { MO1 } & \boldsymbol{\Gamma}_{01}(P \psi)=(P \psi) .
\end{array}
$$

In this case, there is a corresponding alternative to the super-exceptional isometry (4.6) given by

$$
v_{9}^{\mathrm{ex}_{s}}:=\delta_{9}^{a} v_{a}-(1+s) \frac{1}{2} \epsilon_{01}^{a_{1} a_{2}} v_{a_{1} a_{2}}+\chi^{\alpha} v_{\alpha} .
$$

With this alternative brane configuration and alternative super-exceptional isometry, all of the following constructions go through for all of $s \in \mathbb{R} \backslash\{0\}$, including $s=-6$; but then there is no value of $s$ for which the leading term of the super-exceptional Perry-Schwarz Lagrangian equals the original bosonic Perry-Schwarz Lagrangian, i.e., what fails is item (ii) of proposition 5.1 below. This does not mean that this alternative case is not of interest, but its interpretation will need to be discussed elsewhere.

We record the following basic fact:

Lemma 4.11 (Vanishing bilinears on $\frac{1}{2} \mathrm{M} 5$ spacetime). The following bispinor pairings vanish identically

$$
\begin{array}{lll}
\left(\overline{P_{\mathbf{8}} \psi}\right) \Gamma_{5}\left(P_{\mathbf{8}} \psi\right) & =0 & \\
\left(\overline{P_{\mathbf{8}} \psi}\right) \Gamma_{a_{1} a_{2}}\left(P_{\mathbf{8}} \psi\right) & =0 & \text { for } a_{1}, a_{2} \neq 5, \\
\left(\overline{P_{\mathbf{8}} \psi}\right) \Gamma_{a_{1} \cdots a_{5}}\left(P_{\mathbf{8}} \psi\right)=0 & \text { if one } a_{i}=5 .
\end{array}
$$

Proof. Consider the following computation, for any $a_{i} \in\left\{1,2,3,4,5^{\prime}, 5,6,7,8,9\right\}$ :

$$
\begin{aligned}
\left(\overline{P_{\mathbf{8}} \psi}\right) \Gamma_{a_{1} \cdots a_{n}}\left(P_{\mathbf{8}} \psi\right) & =\left(P_{\mathbf{8}} \psi\right)^{\dagger} \Gamma_{0 a_{1} \cdots a_{n}}\left(P_{\mathbf{8}} \psi\right) \\
& =\left(P_{\mathbf{8}} \psi\right)^{\dagger} \Gamma_{0 a_{1} \cdots a_{n}} \boldsymbol{\Gamma}_{5}\left(P_{\mathbf{8}} \psi\right) \\
& = \pm\left(P_{\mathbf{8}} \psi\right)^{\dagger} \boldsymbol{\Gamma}_{5} \Gamma_{0 a_{1} \cdots a_{n}}\left(P_{\mathbf{8}} \psi\right) \\
& =\sigma \cdot\left(\boldsymbol{\Gamma}_{5} P_{\mathbf{8}} \psi\right)^{\dagger} \Gamma_{0 a_{1} \cdots a_{n}}\left(P_{\mathbf{8}} \psi\right) \\
& =\sigma \cdot\left(P_{\mathbf{8}} \psi\right)^{\dagger} \Gamma_{0 a_{1} \cdots a_{n}}\left(P_{\mathbf{8}} \psi\right) \\
& =\sigma \cdot\left(\overline{P_{\mathbf{8}} \psi}\right) \Gamma_{0 a_{1} \cdots a_{n}}\left(P_{\mathbf{8}} \psi\right) .
\end{aligned}
$$


Here the first line is the definition of the Dirac adjoint, the second line uses that $\boldsymbol{\Gamma}_{5}$ is the identity on the projected spinors, by definition. In the third step we commute $\boldsymbol{\Gamma}_{5}=i \Gamma_{5}$ with $\Gamma_{0 a_{1} \cdots a_{n}}$, thereby picking up a sign

$$
\sigma=\left\{\begin{array}{cc}
+1 \mid & \text { odd number of } a_{i} \mathrm{~s} \neq 5 \\
-1 \mid & \text { even number of } a_{i} \mathrm{~s} \neq 5
\end{array}\right.
$$

Finally we use $\left(\boldsymbol{\Gamma}_{5}\right)^{\dagger}=\boldsymbol{\Gamma}_{5}$ from (3.1) to absorb the $\boldsymbol{\Gamma}_{5}$ again, this time into the left spinor factor. Hence the expression we started with equals its product with $\sigma$, and so vanishes when $\sigma=-1$, hence when $\Gamma_{a_{1} \cdots a_{n}}$ has an even number of indices differing from 5 .

For the M-brane cochains, and in terms of super-exceptional embedding, this means the following:

Lemma 4.12 (Pullback of M-brane cocycles to $\frac{1}{2} \mathrm{M} 5$ ). For the M-brane cocycles (1.2), pulled back along the super-embedding of $\frac{1}{2}$ M5-embedding $i$ in (4.12), we have

$$
\begin{aligned}
& i^{*} \mu_{\mathrm{M} 2}=e^{5} \wedge \iota_{v_{5}} i^{*}\left(\mu_{\mathrm{M} 2}\right), \\
& i^{*} \mu_{\mathrm{M} 5}=\left(\mathrm{id}-e^{5} \wedge \iota_{v_{5}}\right) i^{*} \mu_{\mathrm{M} 5},
\end{aligned}
$$

with $\iota_{v_{5}}$ given in (4.5). Hence, for the super-exceptional M-brane cocycles (definition 3.8), we have

$$
\begin{aligned}
& \left(i_{\mathrm{ex}_{s}}\right)^{*}\left(\pi_{\mathrm{ex}_{s}}\right)^{*} \mu_{\mathrm{M} 2}=e^{5} \wedge \iota_{v_{5}^{\mathrm{ex}_{s}}}\left(i_{\mathrm{ex}_{s}}\right)^{*}\left(\pi_{\mathrm{ex}_{s}}\right)^{*} \mu_{\mathrm{M} 2}, \\
& \left(i_{\mathrm{ex}_{s}}\right)^{*}\left(\pi_{\mathrm{ex}_{s}}\right)^{*} \mu_{\mathrm{M} 5}=\left(\mathrm{id}-e^{5} \wedge \iota_{v_{5}}^{\mathrm{ex}_{s}}\right)\left(i_{\mathrm{ex}_{s}}\right)^{*}\left(\pi_{\mathrm{ex}_{s}}\right)^{*} \mu_{\mathrm{M} 5} .
\end{aligned}
$$

Proof. By lemma 4.8, the pullbacks along $i$ and $i_{\mathrm{ex}_{s}}$ act on the spinors by applying the projection $P_{\mathbf{8}}$. Hence lemma 4.11 applies to the spinor bilinears after restriction and says that all summands in the pullbacks of the M-brane cochains that do not contain an index $=5$ (for the M2-brane cocycle) or do contain an index $=5$ (for the M5-brane cochain) vanish. This is just what is expressed by the projection operators in (4.16).

Example 4.13 (M-brane cochains pulled back to super-exceptional brane loci). The pullbacks of the super M2-brane cocycle $\mu_{\mathrm{M} 2}$ and of the super M5-brane cochain $\mu_{\mathrm{M} 5}(1.2)$ horizontally along the embeddings in (4.12) are as follows:

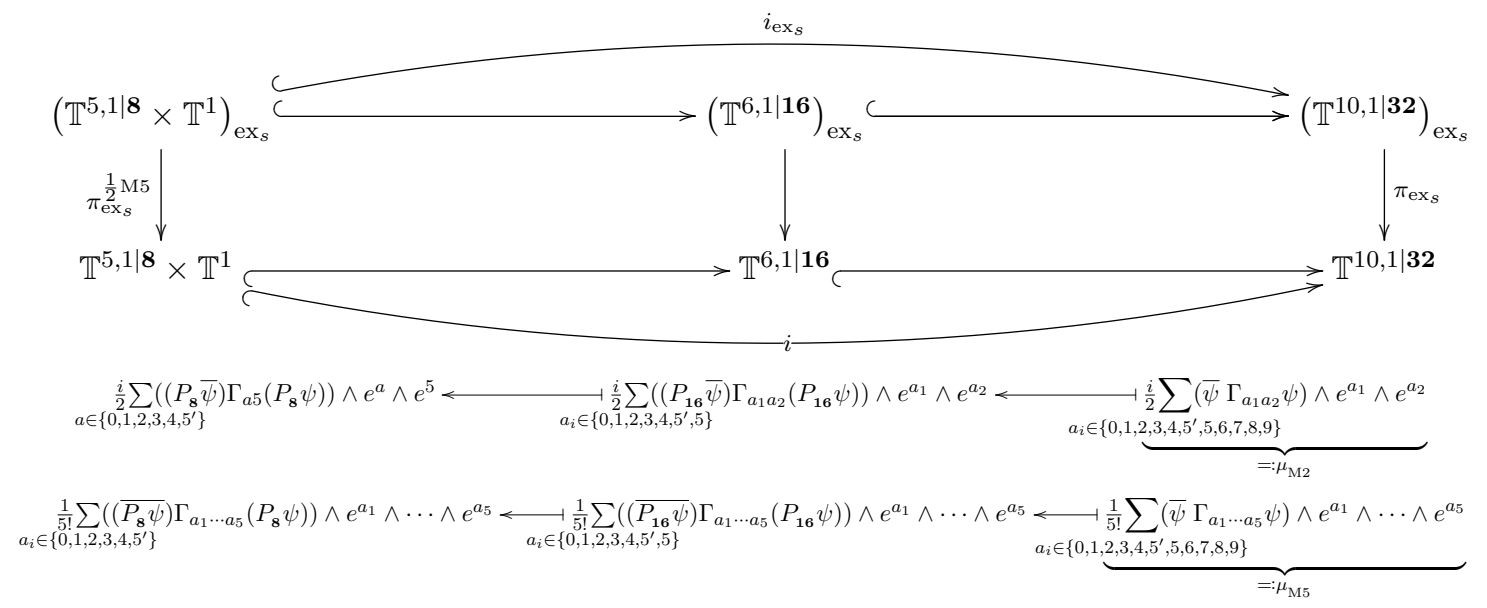


Here $P_{16}$ and $P_{8}$ are the spinor projections from definition 4.1, and on the far left we used lemma 4.12 to recognize that the M2-brane cocycle on the $\frac{1}{2} \mathrm{M} 5$-locus retains only the summands proportional to $e^{5}$, while the M5-brane cochain on the $\frac{1}{2} \mathrm{M} 5$-locus retains only the summands not proportional to $e^{5}$. Notice that the vertical pullback is syntactically the identity, due to (3.3). This makes manifest that the vertical pullback to the exceptional spacetimes intertwines the contraction operations for $v_{5}(4.5)$ and for $v_{5}^{\mathrm{ex}_{s}}(4.6)$ :

$$
\left(\pi_{\mathrm{ex}_{s}}^{\frac{1}{2} \mathrm{M} 5}\right)^{*} \circ \iota_{v_{5}}=\iota_{v_{5}}^{\mathrm{ex}_{s}} \circ\left(\pi_{\mathrm{ex}_{s}}^{\frac{1}{2} \mathrm{M} 5}\right)^{*} .
$$

Since we also have

$$
i^{*} \circ \iota_{v_{5}}=\iota_{v_{5}} \circ i^{*}
$$

this implies

$$
\left(i \circ \pi_{\mathrm{ex}_{s}}^{\frac{1}{2} \mathrm{M} 5}\right)^{*} \circ \iota_{v_{5}}=\iota_{v_{5}}^{\mathrm{ex}_{s}} \circ\left(i \circ \pi_{\mathrm{ex}_{s}}^{\frac{1}{2} \mathrm{M} 5}\right)^{*} .
$$

\section{Super-exceptional Perry-Schwarz \& Yang-Mills Lagrangians}

In proposition 5.1 we find a natural super-exceptional pre-image of the bosonic PerrySchwarz Lagrangian, recorded as definition 5.2 below. This allows us to extract the supercomponents (in proposition 5.3 below) and identify the super-exceptional M-theory avatar of the gaugino field (remark 5.4 below). We also find the super-exceptional lift of the topological Yang-Mills Lagrangian (definition 5.5 below) and its relation to the superexceptional Perry-Schwarz Lagrangian (lemma 5.6 below). This plays a crucial role when we unify all this super-exceptional data in section 6. Then we show (proposition 5.9 below) that the super-exceptional Perry-Schwarz Lagrangian arises as the trivialization of the super-exceptional M5-brane cocycle restricted along the super-embedding of the $\frac{1}{2} \mathrm{M} 5$ spacetime and compactified on the $\mathrm{M} / \mathrm{HW}$-theory circle (definition 5.7 below). This is a key ingredient in the full super-embedding theorem 7.3 further below in section 7

We start with identifying the super-exceptional lift of the PS Lagrangian.

Proposition 5.1 (Super-exceptional lift of bosonic 2-flux and PS Lagrangian). Consider $\Sigma_{\mathrm{bos}}:=\mathbb{R}^{5,1} \times \mathbb{R}^{1}$ and a section $\sigma$ of the super-exceptional $\frac{1}{2}$ M5-brane spacetime projection (4.12)
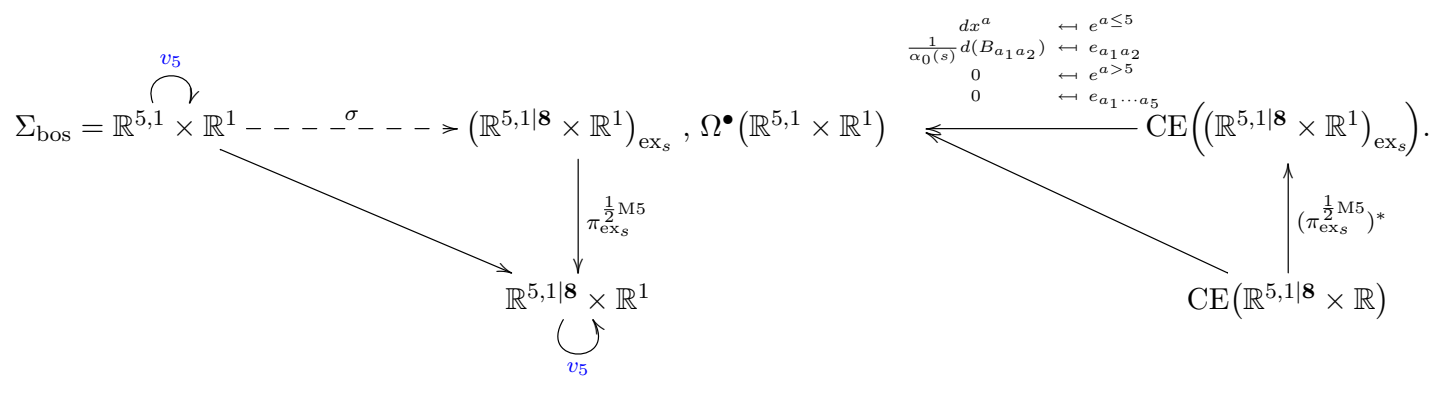

(i) If $\sigma$ is such that the normal forms $e^{a>5}$ and the 5-index forms $e_{a_{1} \cdots a_{5}}$ pull back to zero, as shown above on the right, then the pullback of the contraction of the transgression 
element $H_{\mathrm{ex}_{s}}$ (proposition 3.5) with the lifted vector field $v_{5}^{\mathrm{ex}_{s}}$ (proposition 4.4) is the bosonic 2-form flux F (2.18):

$$
\begin{aligned}
& \Omega^{\bullet}\left(\Sigma_{\text {bos }}\right) \longleftarrow \sigma^{*}-\operatorname{CE}\left(\left(\mathbb{R}^{5,1 \mid \mathbf{8}} \times \mathbb{R}^{1}\right)_{\mathrm{ex}_{s}}\right) \\
& \underbrace{\overbrace{\iota_{5} H}^{=\mathcal{F}}-\mathcal{L}_{v_{5}} B^{\text {hor }}}_{=F} \longleftarrow \underbrace{\iota_{v_{5}^{\mathrm{ex}_{s}} H_{\mathrm{ex}_{s}}}}_{=: F_{\mathrm{ex}_{s}}}
\end{aligned}
$$

where on the left $H:=d\left(B_{a_{2} a_{3}}\right) \wedge d x^{a_{2}} \wedge d x^{a_{3}}$ denotes the plain $H$-flux (3.6) and $F$ its induced 2-form flux (2.18) according to lemma 2.3; and hence on the right we find a super-exceptional pre-image $F_{\mathrm{ex}_{s}}$ of the 2-form flux.

(ii) If, moreover, $s=-3$ (as in example 3.6), we have

$$
\begin{gathered}
\Omega^{\bullet}\left(\Sigma_{\mathrm{bos}}\right) \longleftarrow \sigma^{*} \\
\underbrace{-\frac{1}{2}\left(\iota_{v_{5}} H-\mathcal{L}_{v_{5}} B^{\mathrm{hor}}\right) \wedge H \wedge d x^{5}}_{=\mathrm{I}^{\mathrm{PS}} \wedge d x^{5}} \leftarrow \underbrace{\mathrm{CE}\left(\left(\mathbb{R}^{5,1 \mid 8} \times \mathbb{R}^{1}\right)_{\mathrm{ex}_{s}}\right)}_{=: \mathrm{I}_{\mathrm{exs}}^{\mathrm{S}} \wedge e^{5}}
\end{gathered}
$$

where on the left we have the Perry-Schwarz Lagrangian (2.24), and hence on the right we find a super-exceptional pre-image $\mathbf{L}_{\mathrm{ex}_{s}}^{\mathrm{PS}}$.

Proof. By the assumption that $\sigma^{*} e_{a_{1} \cdots a_{5}}=0$, and since the odd forms $\sigma^{*} \psi$ and $\sigma^{*} \eta$ vanish after pullback to the bosonic space $\mathbb{R}^{5,1} \times \mathbb{R}^{1}$, we find from (3.6) by direct computation that

$$
\begin{aligned}
\sigma^{*}\left(\iota_{v_{5}^{\text {ex }}} H_{\mathrm{ex}_{s}}\right) & =\alpha_{0}(s) \cdot \sigma^{*}\left(\iota_{v_{5}^{\mathrm{ex}}} e_{a_{2} a_{3}} \wedge e^{a_{2}} \wedge e^{a_{3}}\right) \\
& =\alpha_{0}(s) \cdot \sigma^{*}\left(-2 e_{5 a_{3}} \wedge e^{5} \wedge e^{a_{3}}\right) \\
& =-2\left(d B_{5 a_{3}}\right) \wedge d x^{5} \wedge d x^{a_{3}} \\
& =-2\left(\partial_{v^{a_{1}}} B_{5 a_{3}}\right) \wedge d x^{a_{1}} \wedge d x^{5} \wedge d x^{a_{3}} \\
& =\iota_{v_{5}}\left(\partial_{a_{1}} B_{a_{2} a_{3}} \wedge d x^{a_{1}} \wedge d x^{a_{2}} \wedge d x^{a_{3}}\right)-\mathcal{L}_{v^{5}} \sum_{a_{2}, a_{3} \neq 5} B_{a_{2} a_{3}} \wedge d x^{a_{2}} \wedge d x^{a_{3}} \\
& =\iota_{v_{5}}(\underbrace{d B_{a_{2} a_{3}} \wedge d x^{a_{2}} \wedge d x^{a_{3}}}_{=H})-\mathcal{L}_{v^{5}} \sum_{a_{2}, a_{3} \neq 5} B_{a_{2} a_{3}} \wedge d x^{a_{2}} \wedge d x^{a_{3}} \\
& =\iota_{v_{5}} H-\mathcal{L}_{v_{5}} B^{\mathrm{hor}} .
\end{aligned}
$$

This proves the first statement. For the second, it is now sufficient to observe with (3.10) that, by the assumption $s=-3$, we have in the present case $\sigma^{*} H_{\mathrm{ex}_{s}}=H$. Hence the second claim now follows directly from the first.

Definition 5.2 (Super-exceptional (dual) 2-flux and PS Lagrangian). On the superexceptional $\frac{1}{2}$ M5 spacetime $\left(\mathbb{R}^{5,1 \mid 8} \times \mathbb{R}^{1}\right)_{\mathrm{ex}_{s}}$ from definition 4.6 define the following forms in $\mathrm{CE}\left(\left(\mathbb{R}^{5,1 \mid \mathbf{8}} \times \mathbb{R}^{1}\right)_{\mathrm{ex}_{s}}\right)$ : 
(i) The super-exceptional 2-flux and dual super-exceptional 2-flux, respectively:

$$
F_{\mathrm{ex}_{s}}:=\iota_{v_{5}^{\operatorname{ex}_{s}}}\left(i_{\mathrm{ex}_{s}}\right)^{*} H_{\mathrm{ex}_{s}}, \quad \widetilde{F}_{\mathrm{ex}_{s}}:=\left(i_{\mathrm{ex}_{s}}\right)^{*} H_{\mathrm{ex}_{s}}-e^{5} \wedge F_{\mathrm{ex}_{s}},
$$

where $H_{\mathrm{ex}_{s}}$ is from proposition 3.5 and $\iota_{v_{5}^{\mathrm{ex}}}$ from proposition 4.4.

(ii) The super-exceptional PS Lagrangian:

$$
\mathbf{L}_{\mathrm{ex}_{s}}^{\mathrm{PS}}:=-\frac{1}{2} F_{\mathrm{ex}_{s}} \wedge \widetilde{F}_{\mathrm{ex}_{s}} .
$$

With the exceptional pre-image of the bosonic 2-form flux identified, we find the induced supersymmetric completion, keeping in mind the notation deg $=$ (bosonic, fermionic):

Proposition 5.3 (Super 2-flux from super-exceptional 2-flux). For parameter value $s=$ -3, eq. (3.2), the exceptional 2-flux density (5.2) is the sum of the bosonic term plus a fermionic term $F_{(1,1)}$ as follows:

$$
F_{\mathrm{ex}_{s}}=\underbrace{F}_{=: F_{(2,0)}}+\underbrace{\left(\bar{\psi} \Gamma_{a} \chi\right) \wedge e^{a}}_{=: F_{(1,1)}}+\mathcal{O}\left(\left\{e_{a_{1} \cdots a_{5}}\right\}\right) .
$$

Proof. By example 3.6 we have $H_{\mathrm{ex}_{-3}}=H+\left(\bar{\psi} \Gamma_{a} \eta\right) \wedge e^{a}+\mathcal{O}\left(\left\{e_{a_{1} \cdots a_{5}}\right\}\right)$. From this the statement follows by the definition (4.6) of $v_{5}^{\mathrm{ex}_{s}}$ and using the identities $(\overline{P \psi}) \Gamma_{5}(P \chi)=0$ and $(\overline{P \psi}) \Gamma_{56789}(P \chi)=0$ from lemma 4.11 .

Remark 5.4 (Super 2-form gauge field strength and gauginos). The summand

$$
F_{(1,1)}=\left(\bar{\psi} \Gamma_{a} \chi\right) \wedge e^{a}
$$

in (5.5) is exactly the supersymmetic enhancement of the gauge curvature in 10d SYM, with $\chi$ identified as the gaugino field ([117], [7, (4.14)], [17, (2.27)]). But by the last line of (4.6), $\chi$ is the component of the super-exceptional lift $v_{5}^{\text {ex }_{s}}$ of the isometry along $S_{\mathrm{HW}}^{1}$ in the fermionic direction defined by the extra super-exceptional 1-form $\eta$ (3.3)

$$
\iota_{v_{5}^{\mathrm{ex}}}: \eta \mapsto \chi
$$

In this way, it is the extra super-exceptional fermionic coordinate $\eta$ which is the avatar on the super-exceptional M-theory spacetime of what becomes the gaugino field upon compactification to heterotic M-theory on $S_{\mathrm{HW}}^{1}$.

Note that an approximate construction of the $11 \mathrm{~d}$ gravitino in the context of $E_{8}$ gauge theory as a condensate of the gauge theory fields is given in [37].

From proposition 5.1 it is clear that we have a super-exceptional lift of the topological Yang-Mills Lagrangian $\mathbf{L}^{\mathrm{tYM}}=-\frac{1}{2} F \wedge F(2.31)$ :

Definition 5.5. The super-exceptional topological Yang-Mills Lagrangian is the wedge square of the super-exceptional 2-flux (5.3) from definition 5.2:

$$
\mathbf{L}_{\mathrm{ex}_{s}}^{\mathrm{tYM}}:=-\frac{1}{2} F_{\mathrm{ex}_{s}} \wedge F_{\mathrm{ex}_{s}} .
$$


Lemma 5.6 (Super-exceptional topological Yang-Mills as compactification of super-exceptional Perry-Schwarz). The contraction of the super-exceptional Perry-Schwarz Lagrangian (5.4) with the super-exceptional isometry $v_{5}^{\mathrm{ex}_{s}}(4.6)$ along $S_{\mathrm{HW}}^{1}$ is the superexceptional topological Yang-Mills Lagrangian (5.6):

$$
\iota_{v_{5}^{\mathrm{ex}}} \mathbf{L}_{\mathrm{ex}_{s}}^{\mathrm{PS}}=\mathbf{L}_{\mathrm{ex}_{s}}^{\mathrm{tYM}} .
$$

Proof. We need to show that

$$
\iota_{v_{5}^{\mathrm{ex}}}\left(\widetilde{F}_{\mathrm{ex}_{s}} \wedge F_{\mathrm{ex}_{s}}\right)=F_{\mathrm{ex}_{s}} \wedge F_{\mathrm{ex}_{s}} .
$$

But this follows directly from the definitions and the the fact that the contraction is a graded derivation of degree ( -1 , even), hence in particular nilpotent. Indeed, we have

$$
\begin{aligned}
\iota_{v_{5}^{\mathrm{ex}}}\left(\widetilde{F}_{\mathrm{ex}_{s}} \wedge F_{\mathrm{ex}_{s}}\right) & =\iota_{v_{5}^{\mathrm{ex}}}\left(\left(H_{\mathrm{ex}_{s}}-e^{5} \wedge \iota_{v_{5}^{\mathrm{ex}_{s}}} H_{\mathrm{ex}_{s}}\right) \wedge \iota_{v_{5}^{\mathrm{ex}}} H_{\mathrm{ex}_{s}}\right) \\
& =\left(\iota_{v_{5}^{\mathrm{ex}_{s}}} H_{\mathrm{ex}_{s}}\right) \wedge\left(\iota_{v_{5}^{\mathrm{ex}_{s}}} H_{\mathrm{ex}_{s}}\right) \\
& =F_{\mathrm{ex}_{s}} \wedge F_{\mathrm{ex}_{s}} .
\end{aligned}
$$

In order to see the super-exceptional Perry-Schwarz Lagrangian arise from the superexceptional M5-brane cocycle, we now first consider the M5-brane sigma-model wrapped on the $S_{\mathrm{HW}}^{1}$-fiber (see remark 4.2) of super-exceptional M-theory spacetime (definition 3.3). By the general rules of (double-)dimensional reduction of super $p$-brane cocycles [43, section 3], [44, section 3], [19, section 2.2], this means that we are to contract the plain M5-brane cocycle (1.2) with the vector field corresponding to the flow along this fiber, hence with $v_{5}$ (4.5). The following definition lifts this situation to super-exceptional spacetime.

Definition 5.7 (Super-exceptional circle compactification of M5 cocycle). The compactification on $S_{\mathrm{HW}}^{1}$ of the super-exceptional M5-brane cocycle $\mathbf{d L}_{\mathrm{ex}_{s}}^{\mathrm{WZ}}$ (definition 3.8) pulled back along the super-exceptional embedding $i_{\mathrm{ex}_{s}}$ (4.12) to the normally thickened superexceptional $\frac{1}{2} \mathrm{M} 5$-spacetime (definition 4.6) is its contraction with the super-exceptional lift $v_{5}^{\text {ex }}$ (proposition 4.4) of the vector field $v_{5}$ along $S_{\mathrm{HW}}^{1}(4.2)$ :

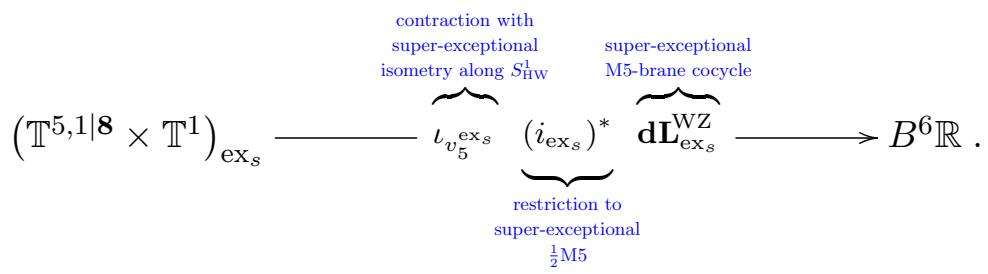

Notice that this is indeed still a cocycle, in that it is closed, $d\left(\iota_{v_{5}^{\mathrm{ex}}}\left(i_{\mathrm{ex}_{s}}\right)^{*} \mathbf{d L}_{\mathrm{ex}_{s}}^{\mathrm{WZ}}\right)=0$, by (4.8) in proposition 4.4.

Remark 5.8 (Cocycle for M5/S as D4 + KK). From the type I' perspective on the $\frac{1}{2} \mathrm{M} 5$ locus (as in remark 4.2) the compactified M5-cocycle of definition 5.7 would be that of a D4-brane inside a $\frac{1}{2} \mathrm{NS} 5=\mathrm{NS} 5 \cap \mathrm{O} 8$, by the general rules of dimensional reduction of brane cocycles [43, section 3], [44, section 3], [19, section 2.2]. However, since we need not consider double dimensional reduction, in that the super-exceptional coordinate 
functions along $v_{5}$ are still present in the normally thickened $\frac{1}{2}$ M5 locus $\left(\mathbb{R}^{5,1 \mid 8} \times \mathbb{R}^{1}\right)_{\mathrm{ex}_{s}}$ (definition 4.6), fields on this would-be D4 may still depend on the M-theory circle direction, hence have KK-modes along the circle. In this sense, definition 5.7 exhibits the brane cocycle corresponding to the perspective on the M5-brane as a non-perturbative D4-brane with KK-modes included, as considered in [35, 76] (see [77, 3.4.3]).

We now establish the following super-exceptional analog of the super-embedding mechanism.

Proposition 5.9 (PS Lagrangian trivializes compactified M5-cocycle along super-exceptional embedding). The compactification of the restriction of the super-exceptional M5brane cocycle (definition 5.7) along the embedding of the super-exceptional $\frac{1}{2} \mathrm{M} 5$ spacetime (definition 4.6) is trivialized by the super-exceptional PS Lagrangian (5.4):

$$
\iota_{v_{5}^{\mathrm{ex}_{s}}}\left(i_{\mathrm{ex}_{s}}\right)^{*} \mathbf{d L}_{\mathrm{ex}_{s}}^{\mathrm{WZZ}}=d \mathbf{L}_{\mathrm{ex}_{s}}^{\mathrm{PS}} .
$$

Proof. Unravelling the definitions, we have to show that

$$
\iota_{v_{5}^{\mathrm{ex}}}\left(i_{\mathrm{ex}_{s}}\right)^{*}\left(\left(\pi_{\mathrm{ex}_{s}}\right)^{*} \mu_{\mathrm{M} 5}+\frac{1}{2} H_{\mathrm{ex}_{s}} \wedge d H_{\mathrm{ex}_{s}}\right)=d\left(-\frac{1}{2} F_{\mathrm{ex}_{s}} \wedge \widetilde{F}_{\mathrm{ex}_{s}}\right) .
$$

For the first summand on the left, we immediately obtain

$$
\begin{aligned}
\iota_{v_{5}}^{\mathrm{ex}_{s}}\left(i_{\mathrm{ex}_{s}}\right)^{*}\left(\pi_{\mathrm{ex}_{s}}\right)^{*} \mu_{\mathrm{M} 5} & =\left(\pi_{\mathrm{ex}_{s}}^{\frac{1}{2} \mathrm{M} 5}\right)^{*} \underbrace{\left(i^{*} \iota_{v_{5}} \mu_{\mathrm{M} 5}\right)}_{=0} \\
& =0,
\end{aligned}
$$

by lemma 4.12; see example 4.13. For the second summand (or rather twice the second summand, for notational convenience) we compute as follows:

$$
\begin{aligned}
& 2 \iota_{v_{5}}^{\mathrm{ex}_{s}}\left(i_{\mathrm{ex}_{s}}\right) *\left(\frac{1}{2} H_{\mathrm{ex}_{s}} \wedge d H_{\mathrm{ex}_{s}}\right) \\
& =\iota_{v_{5}}^{\mathrm{ex}_{s}}\left(i_{\mathrm{ex}_{s}}\right)^{*}\left(H_{\mathrm{ex}_{s}} \wedge d H_{\mathrm{ex}_{s}}\right) \\
& =\iota_{v_{5}}^{\mathrm{ex}_{s}}\left(\left(\left(i_{\mathrm{ex}_{s}}\right)^{*} H_{\mathrm{ex}_{s}}\right) \wedge\left(\left(i_{\mathrm{ex}_{s}}\right)^{*} d H_{\mathrm{ex}_{s}}\right)\right) \\
& =\left(\iota_{v_{5}}^{\mathrm{ex}_{s}}\left(i_{\mathrm{ex}_{s}}\right)^{*} H_{\mathrm{ex}_{s}}\right) \wedge\left(\left(i_{\mathrm{ex}_{s}}\right)^{*} d H_{\mathrm{ex}_{s}}\right)-\left(\left(i_{\mathrm{ex}_{s}}\right)^{*} H_{\mathrm{ex}_{s}}\right) \wedge\left(\iota_{v_{5}}^{\mathrm{ex}_{s}}\left(i_{\mathrm{ex}_{s}}\right)^{*} d H_{\mathrm{ex}_{s}}\right) \\
& =\left(\iota_{v_{5}}^{\mathrm{ex}_{s}}\left(i_{\mathrm{ex}_{s}}\right)^{*} H_{\mathrm{ex}_{s}}\right) \wedge\left(\left(i_{\mathrm{ex}_{s}}\right)^{*} d H_{\mathrm{ex}_{s}}\right)+\left(\left(i_{\mathrm{ex}_{s}}\right)^{*} H_{\mathrm{ex}_{s}}\right) \wedge\left(d\left(\iota_{v_{5}}^{\mathrm{ex}_{s}}\left(i_{\mathrm{ex}_{s}}\right)^{*} H_{\mathrm{ex}_{s}}\right)\right) \\
& =2\left(\iota_{v_{5}}^{\mathrm{ex}_{s}}\left(i_{\mathrm{ex}_{s}}\right)^{*} H_{\mathrm{ex}_{s}}\right) \wedge\left(\left(i_{\mathrm{ex}_{s}}\right)^{*} d H_{\mathrm{ex}_{s}}\right)-d\left(\left(\left(i_{\mathrm{ex}_{s}}\right)^{*} H_{\mathrm{ex}_{s}}\right) \wedge\left(\iota_{v_{5}}^{\mathrm{ex}_{s}}\left(i_{\mathrm{ex}_{s}}\right)^{*} H_{\mathrm{ex}_{s}}\right)\right) \\
& =2\left(\iota_{v_{5}}^{\mathrm{ex}_{s}}\left(i_{\mathrm{ex}_{s}}\right)^{*} H_{\mathrm{ex}_{s}}\right) \wedge\left(e^{5} \wedge \iota_{v_{5}}^{\mathrm{ex}_{s}}\left(i_{\mathrm{ex}_{s}}\right)^{*} d H_{\mathrm{ex}_{s}}\right)-d\left(\left(\left(i_{\mathrm{ex}_{s}}\right)^{*} H_{\mathrm{ex}_{s}}\right) \wedge\left(\iota_{v_{5}}^{\mathrm{ex}_{s}}\left(i_{\mathrm{ex}_{s}}\right)^{*} H_{\mathrm{ex}_{s}}\right)\right) \\
& =-2 e^{5} \wedge\left(\iota_{v_{5}}^{\mathrm{ex}_{s}}\left(i_{\mathrm{ex}_{s}}\right)^{*} H_{\mathrm{ex}_{s}}\right) \wedge d\left(\iota_{v_{5}}^{\mathrm{ex}_{s}}\left(i_{\mathrm{ex}_{s}}\right)^{*} H_{\mathrm{ex}_{s}}\right)-d\left(\left(\left(i_{\mathrm{ex}_{s}}\right)^{*} H_{\mathrm{ex}_{s}}\right) \wedge\left(\iota_{v_{5}}^{\mathrm{ex}_{s}}\left(i_{\mathrm{ex}_{s}}\right)^{*} H_{\mathrm{ex}_{s}}\right)\right) \\
& =d\left(e^{5} \wedge\left(\iota_{v_{5}}^{\mathrm{ex}_{s}}\left(i_{\mathrm{ex}_{s}}\right)^{*} H_{\mathrm{ex}_{s}}\right) \wedge\left(\iota_{v_{5}}^{\mathrm{ex}_{s}}\left(i_{\mathrm{ex}_{s}}\right)^{*} H_{\mathrm{ex}_{s}}\right)\right)-d\left(\left(\left(i_{\mathrm{ex}_{s}}\right)^{*} H_{\mathrm{ex}_{s}}\right) \wedge\left(\iota_{v_{5}}^{\mathrm{ex}_{s}}\left(i_{\mathrm{ex}_{s}}\right)^{*} H_{\mathrm{ex}_{s}}\right)\right) \\
& =d\left(-\left(\left(i_{\mathrm{ex}_{s}}\right)^{*} H_{\mathrm{ex}_{s}}-e^{5} \wedge \iota_{v_{5}}^{\mathrm{ex}_{s}}\left(i_{\mathrm{ex}_{s}}\right)^{*} H_{\mathrm{ex}_{s}}\right) \wedge\left(\iota_{v_{5}}^{\mathrm{ex}_{s}}\left(i_{\mathrm{ex}_{s}}\right)^{*} H_{\mathrm{ex}_{s}}\right)\right) \\
& =d\left(-\widetilde{F}_{\mathrm{ex}_{s}} \wedge F_{\mathrm{ex}_{s}}\right) \\
& =2 d\left(-\frac{1}{2} \widetilde{F}_{\mathrm{ex}_{s}} \wedge F_{\mathrm{ex}_{s}}\right) \text {. }
\end{aligned}
$$


Here the first step just collects the factors. The second fact uses that pullback is an algebra homomorphism, by definition. The third step uses that contraction with $v_{5}^{\mathrm{ex}_{s}}$ is a graded derivation of bi-degree $(-1$, even). In the fourth step we commute the differential in the second summand, first with the pullback operation (using that pullback is in fact a dgalgebra homomorphism, by definition) and then with the contraction operation, using that the corresponding Lie derivative vanishes, by (4.8) in proposition 4.4. In the fifth step we use that the differential (commutes with pullback, as before, and) is a graded derivation of degree (1, even). In the sixth step we realize the presence of the projection $e^{5} \wedge \iota_{v_{5}}^{\mathrm{ex}_{s}}$ according to lemma 4.12, in view of $d H_{\mathrm{ex}_{s}}=\left(\pi_{\mathrm{ex}_{s}}\right)^{*} \mu_{\mathrm{M} 2}$ (3.5). In the seventh step we again commute the differential with pullback and with contraction, as before. In the eighth step we again use the derivation property of the differential to collect a total differential, observing that $d e^{5}=0$ holds on the super-exceptional $\frac{1}{2} \mathrm{M} 5$-spacetime, by lemma 4.11. In the ninth step we collect terms and identify, in the tenth step, the super-exceptional 2-flux and its dual, from definition 5.2. Finally, in the last step we split off the factor of 2 again, just for emphasis.

As a corollary we observe the following:

Proposition 5.10 (Super-exceptional YM-Lagrangian is closed and horizontal). The super-exceptional topological Yang-Mills Lagrangian (definition 5.5) is

(i) closed: $d \mathbf{L}_{\mathrm{ex}_{s}}^{\mathrm{YM}}=0$;

(ii) super-exceptionally horizontal (definition 4.5$): \iota_{v_{5}}^{\operatorname{ex}_{s}} \mathbf{L}_{\mathrm{ex}_{s}}^{\mathrm{YM}}=0$.

Proof. For the first statement, we may compute as follows:

$$
\begin{aligned}
d \mathbf{L}_{\mathrm{ex}_{s}}^{\mathrm{tYM}} & =d \iota_{v_{5}}^{\mathrm{ex}_{s}} \mathbf{L}_{\mathrm{ex}_{s}}^{\mathrm{PS}} \\
& =-\iota_{v_{5}}^{\mathrm{ex}_{s}} d \mathbf{L}_{\mathrm{ex}_{s}}^{\mathrm{PS}} \\
& =\underbrace{\iota_{v_{5}}^{\mathrm{ex}_{s}} \iota_{v_{5}}^{\mathrm{ex}_{s}}}_{=0}\left(i_{\mathrm{ex}_{s}}\right)^{*} \mathbf{d} \mathbf{L}_{\mathrm{ex}_{s}}^{\mathrm{WZ}} \\
& =0,
\end{aligned}
$$

where we used first lemma 5.6, then (4.8) from lemma 4.4 and then proposition 5.9, and finally, under the brace, we observe that contraction with elements in degree (1, even) is nilpotent. This nilpotency also directly implies the second statement, by lemma 5.6.

\section{Super-exceptional equivariance along M-theory circle}

We show (theorem 6.9 below) that the super-exceptional Perry-Schwarz Lagrangian and the super-exceptional topological Yang-Mills Lagrangian unify with the super-exceptional M5 WZ curvature term into the Borel-equivariant enhancement of the super-exceptional M5brane cocycle with respect to the super-exceptional $S_{\mathrm{HW}}^{1}$ isometry left-induced to an $\Omega S_{\mathrm{HW}}^{2}$ action on the super-exceptional $\frac{1}{2} \mathrm{M} 5$-spacetime (definition 6.8). In order to put this in 
perspective, we first show (proposition 6.6 below) that, similarly, the little-string-extended $D=6, \mathcal{N}=(1,1)$, superspacetime carries an $\Omega S^{2}$-action whose homotopy quotient is the $D=5, \mathcal{N}=2$, superspacetime.

To set the scene, we first recall how homotopy quotients are represented in rational cohomology by (Borel-) equivariant de Rham cohomology. From general homotopy theory we need the following two basic facts (see [86]). For any kind of higher geometric spaces (here, rational super spaces), we have:

(i) Forming based loop spaces is an equivalence from pointed connected spaces to $\infty$ groups, whose inverse is the classifying space construction ${ }^{2}$

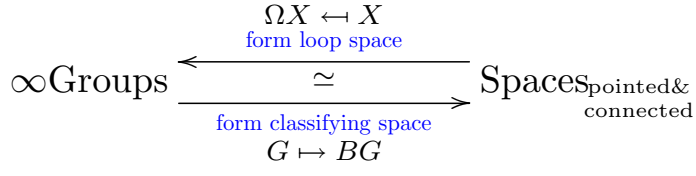

(ii) For $X$ a space and $G$ an $\infty$-group, an $\infty$-action $\rho$ of $G$ on $X$ is equivalently a homotopy fiber sequence of the following form

$$
X \stackrel{\operatorname{hofib}\left(p_{\rho}\right)}{\longrightarrow} X / / G \stackrel{p_{\rho}}{\longrightarrow} B G,
$$

which then exhibits the space in the middle as the homotopy quotient of $X$ by the $\infty$-action $\rho$.

To prepare for proposition 6.6 and theorem 6.9 below, we now consider a sequence of examples of homotopy quotients of rational super spaces as in (6.2). In the following diagrams we always show the systems of spaces on the left with their super dgc-algebra (FDA) models shown on the right. Throughout we use that homotopy pullbacks of super spaces are modeled by pushouts of semi-free super dgc-algebras (FDAs) as soon as the morphism pushed out along is a cofibration in that it exhibits iterated addition of generators. For more background see, for instance, [19, 43, 53, 62].

Example 6.1 (Rational $S^{1}$-equivariant cohomology and Cartan model). Let $X$ be any rational super-space of finite type, hence $\mathrm{CE}(\mathfrak{l} X)$ any finitely generated super-dgc algebra, with differential to be denoted $d_{X}$, and equipped with a graded derivation $\iota_{v}$ of degree $(-1$, even $)$ such that the corresponding Lie derivative vanishes identically:

$$
\mathrm{CE}(\mathfrak{l} X) \stackrel{\iota_{v}}{\longrightarrow} \mathrm{CE}(\mathfrak{l} X) \quad \mathcal{L}_{v}:=\left[d_{X}, \iota_{v}\right]=0 .
$$

The homotopy quotient by the corresponding rational $S^{1}$-action as in (6.2) is given by
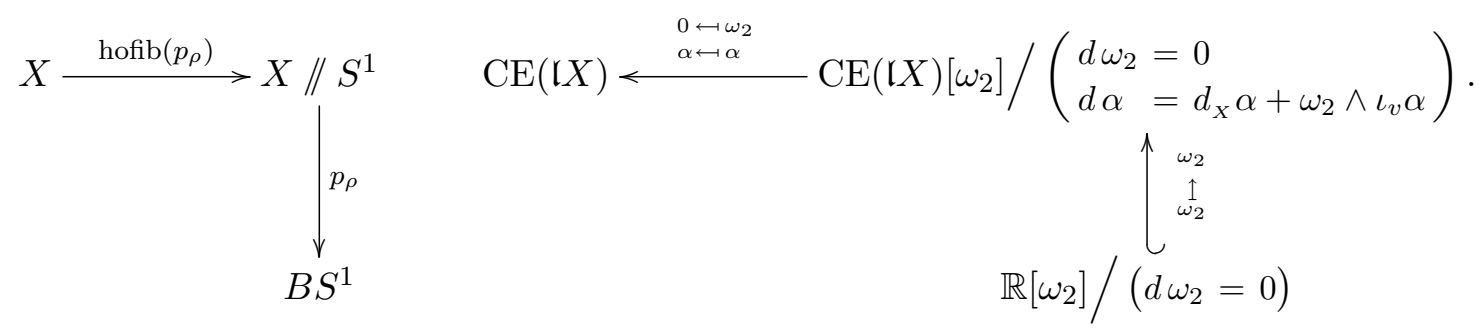

\footnotetext{
${ }^{2}$ For a connected space the choice of basepoint is unique up to homotopy and hence not a choice, up to homotopy.
} 
This is the algebraic structure of the Cartan model for $G$-equivariant Borel cohomology (see e.g. [81, section 5], [55, 82]), here for $G=S^{1}$.

Example 6.2 (Complex Hopf fibration). The complex Hopf fibration $h_{\mathbb{C}}$ realizes the 2sphere $S^{2}$ as the homotopy quotient of the 3 -sphere by an action which is classified by the canonical comparison map from $B \Omega S^{2}$ to $B S^{1}$ :

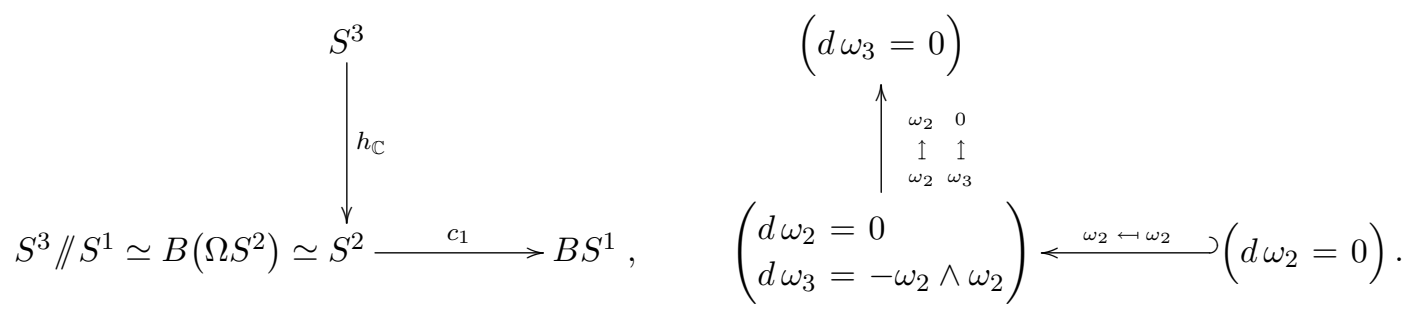

Notice that the classifying map $c_{1}$ here exhibits, by (6.1), a canonical comparison homomorphism of $\infty$-groups

$$
\Omega S^{2} \stackrel{\Omega c_{1}}{\longrightarrow} S^{1}
$$

This loop $\infty$-group of the 2 -sphere, $\Omega S^{2}$, is an $\infty$-group very similar to but just slightly richer than the plain circle.

Example 6.3 (Left-induced $\Omega S^{2}$-equivariant cohomology). This means that an $\infty$-action by $S^{1}$ as in example 6.1 left-induces an $\infty$-action by $\Omega S^{2}$, with its homotopy quotient fiber sequence (6.2) given by homotopy pullback along (6.6), as shown in the following:

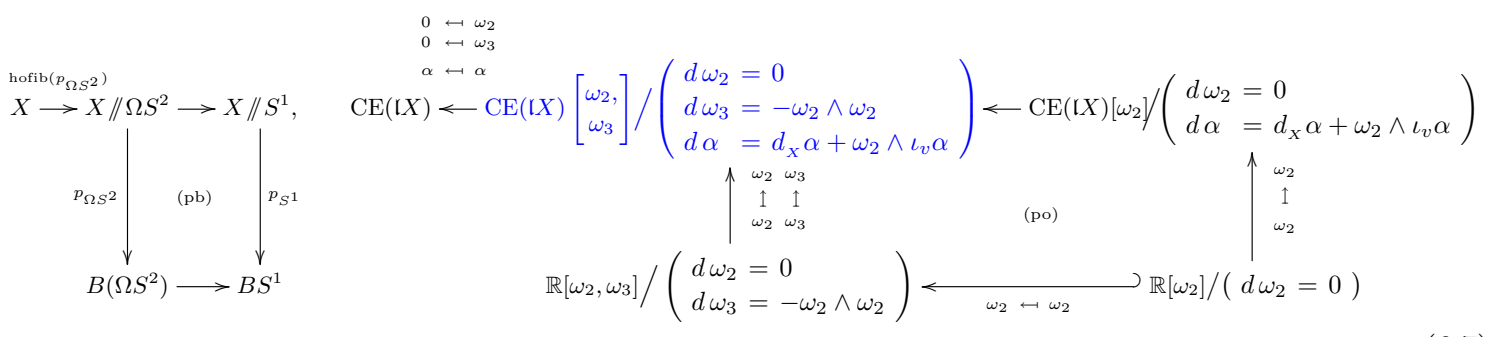

The resulting dgc-algebra, shown in blue, is much like the Cartan model for $S^{1}$-equivariant cohomology as in example 6.1, except that here all even powers of the generator $\omega_{2}$ in bi-degree (2, even), which classifies the circle action, are trivialized in cohomology, by the new generator $\omega_{3}$ in bi-degree (3, even).

Lemma 6.4 ( $\Omega S^{2}$-equivariant cocycles). Rational cocycles on a homotopy quotient $X / / \Omega S^{2}$ for an $\Omega S^{2}$-action that is left-induced according to example 6.3 from an $S^{1}$-action as in example 6.1 are, if they are at most linear in the generator $\omega_{2}$, precisely given by pairs consisting of a cocycle $\alpha$ on $X$ and a trivialization $\beta$ of its contraction with $v$ :

$$
d\left(\alpha-\omega_{2} \wedge \beta-\omega_{3} \wedge \iota_{v} \beta\right)=0 \quad \text { if } d_{X} \alpha=0 \text { and } d_{X} \beta=\iota_{v} \alpha .
$$

Proof. To see that cocycles of this form are closed, we compute as follows, directly unwinding the definitions, where the three lines correspond to application of the three summands 
of the differential $d=d_{X}+\omega_{2} \wedge \iota_{v}+d_{S^{2}}$ :

$$
\begin{aligned}
d\left(\alpha-\omega_{2} \wedge \beta-\omega_{3} \wedge \iota_{v} \beta\right)= & \underbrace{d_{X} \alpha}_{=0}-\omega_{2} \wedge \underbrace{d_{X} \beta}_{=\iota_{v} \alpha}-\omega_{3} \wedge \underbrace{\iota_{v} d_{X} \beta}_{=\iota_{v} \iota_{v} \alpha=0} \\
& +\omega_{2} \wedge \iota_{v} \alpha-\omega_{2} \wedge \omega_{2} \wedge \iota_{v} \beta+\omega_{3} \wedge \omega_{2} \wedge \underbrace{\iota_{v} \iota_{v} \beta}_{=0} \\
& +\omega_{2} \wedge \omega_{2} \wedge \iota_{v} \beta \\
= & 0 .
\end{aligned}
$$

Conversely, reading this same equation as a condition for the vanishing of the coefficients of the products of generators shows that every cocycle of the form on the left of (6.8) satisfies the conditions shown on the right.

We next observe, in proposition 6.6 below, that an $\infty$-action by the $\infty$-group $\Omega S^{2}$ (6.6) characterizes the $D=6, \mathcal{N}=(1,1)$, super-spacetime fibered over the $D=5, \mathcal{N}=2$, superspacetime and exhibits the little-string cocycle in $D=6$ as coming from a 2-spherevalued super-cocycle in $D=5$ (hence in rational Cohomotopy in degree 2). To put this in perspective, we first recall the analogous situation in $D=11$ :

Example 6.5 (Rational sphere-valued cocycles for M-branes in $D=11$ ). We have the situation in (6.9) below:

(i) $[39$, remark 4.4 , proposition 4.5$]$ : the $D=11 \mathcal{N}=1$ super Minkowski spacetime (as in definition 3.3) is the rational $S^{1}$-extension of the $D=10 \mathcal{N}=(1,1)$ (i.e. type IIA) superspacetime, classified by the D0-brane cocycle $\mu_{\mathrm{D} 0}$.

(ii) [102, section 2.5], [42], [43, section 2]: on that extension, the super M2/M5-brane cochains $\left(\mu_{\mathrm{M} 2}, 2 \mu_{\mathrm{M} 5}\right)(1.2)$ constitute a super rational $S^{4}$-valued cocycle, i.e., a cocycle in rational Cohomotopy in degree 4.

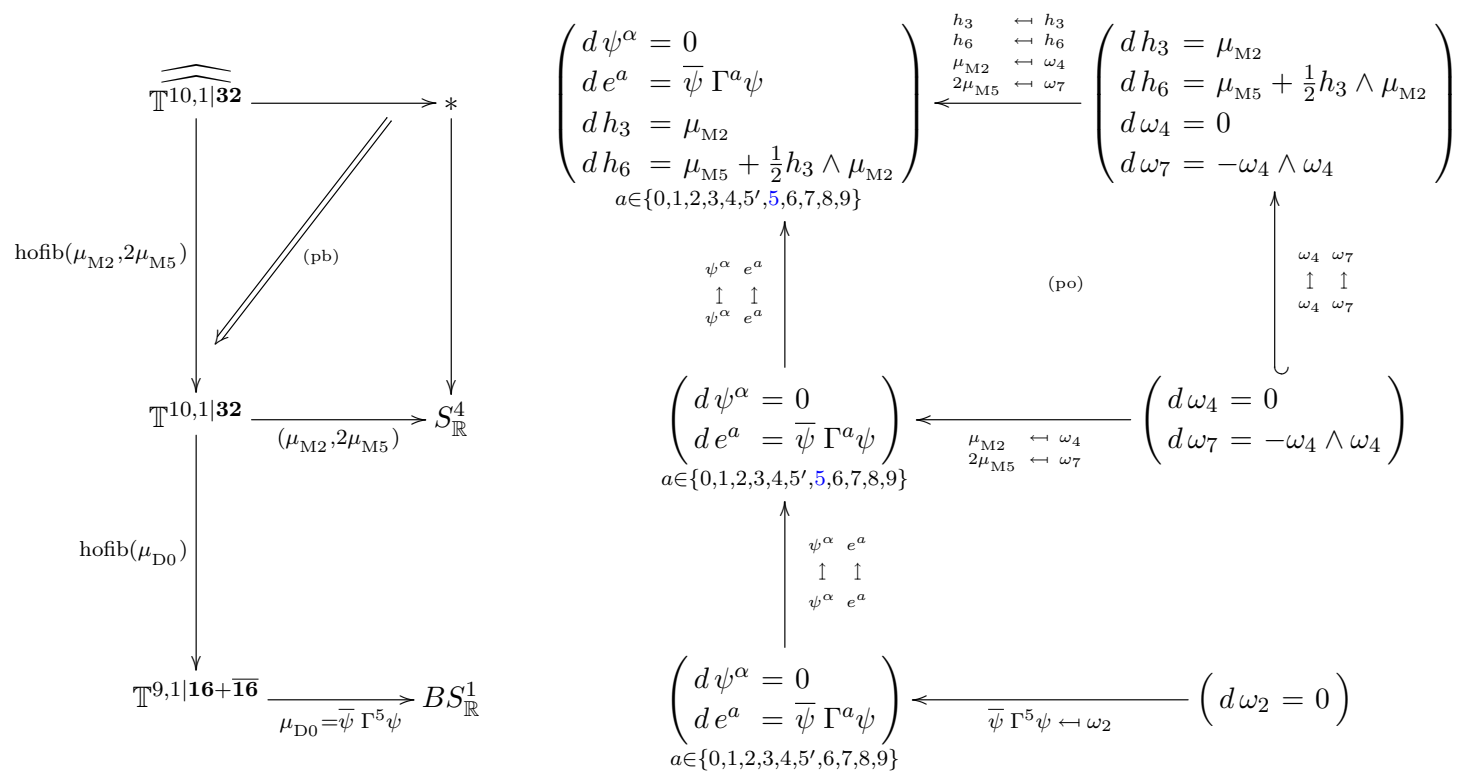


Notice that, when expressed in the canonical super coordinate functions as in (3.4), the bi-fermionic component of the potential $e^{5}$ for the D0-brane cocycle is identified as the super 1-form Ramond-Ramond ( $R R$ ) potential $C_{1}$, i.e., the graviphoton of KK-reduction on $S_{\mathrm{HW}}^{1}$ (e.g. [2, below (51)]):

$$
e^{5}=d x^{5}+\underbrace{\bar{\theta} \Gamma^{5} d \theta}_{=: C_{1}} \stackrel{d}{\longmapsto}(\overline{d \theta}) \Gamma^{5} d \theta=\mu_{\mathrm{D} 0} .
$$

This is analogous to the following situation:

Proposition 6.6 (Fibration of $D=6, \mathcal{N}=(1,1)$, super-spacetime over $D=5$ super-spacetime). The $D=6, \mathcal{N}=(1,1)$, super-spacetime is a rational circle fibration over the $D=5, \mathcal{N}=2$, super-spacetime, which comes via homotopy pullback from the complex Hopf fibration (example 6.2). Moreover, the super-cocycle $\mu_{\mathrm{L} 1}$ for the little string in $6 d$ is induced by a 2-sphere-valued super-cocycle in $D=5$

$$
\mathbb{T}^{4,1 \mid \mathbf{8}+\mathbf{8}} \stackrel{\left(\bar{\psi} \Gamma_{5} \psi, \mu_{\mathrm{L} 1}^{5 d}\right)}{\longrightarrow} S_{\mathbb{R}}^{2},
$$

realizing the little-string extended $D=6$ superspacetime $\widehat{\mathbb{T}^{5,1 \mid \mathbf{8}+\mathbf{8}}}$ as a rational $\Omega S^{2}$-fibration over $D=5$ super-spacetime:
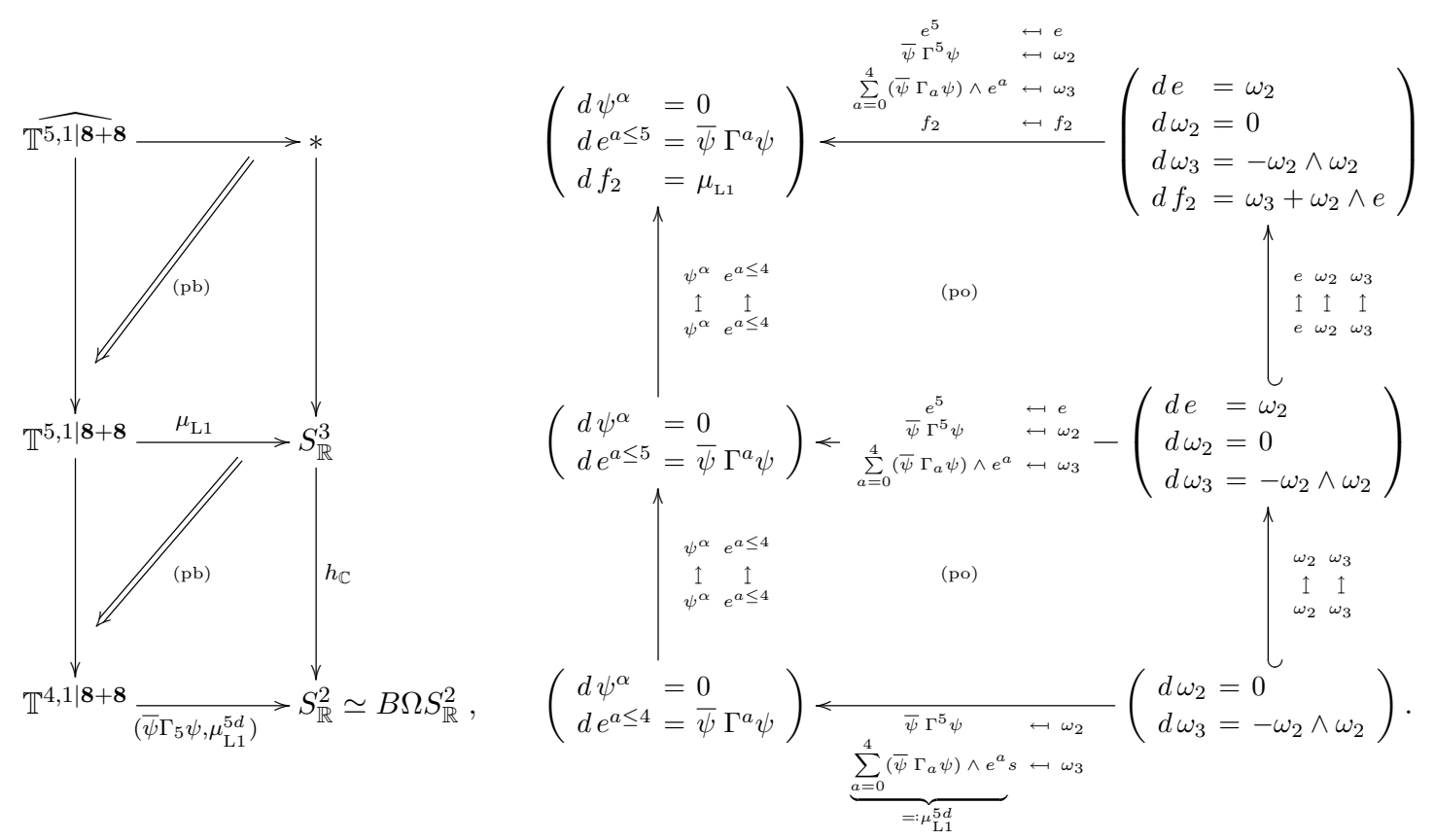

Proof. The statement comes down to proving that

$$
d \mu_{\mathrm{L} 1}=-\left(\bar{\psi} \Gamma_{5} \psi\right)\left(\bar{\psi} \Gamma_{5} \psi\right),
$$

hence that

$$
\sum_{a=0}^{4}\left(\bar{\psi} \Gamma_{a} \psi\right)\left(\bar{\psi} \Gamma^{a} \psi\right)=-\left(\bar{\psi} \Gamma_{5} \psi\right)\left(\bar{\psi} \Gamma_{5} \psi\right)
$$


This is indeed a Fierz identity satisfied by spinors in $D=5$ (e.g. [22, III.5.50]). Equivalently, from the perspective of $D=6$, it reflects the fact that there is the super-cocycle for the little-string (e.g. [22, III.7.14])

$$
\mu_{\mathrm{L} 1}:=\sum_{a=0}^{5}\left(\bar{\psi} \Gamma_{a} \psi\right) \wedge e^{a}=\sum_{a=0}^{4}\left(\bar{\psi} \Gamma_{a} \psi\right) \wedge e^{a}+\left(\bar{\psi} \Gamma_{5} \psi\right) \wedge e^{5}
$$

and that (6.12) is equivalently its closure condition, written with the 5th spatial coordinate summand separated off:

$$
\begin{aligned}
0 & =d \mu_{\mathrm{L} 1} \\
& =\sum_{a=0}^{5}\left(\bar{\psi} \Gamma_{a} \psi\right) d e^{a} \\
& =\sum_{a=0}^{5}\left(\bar{\psi} \Gamma_{a} \psi\right)\left(\bar{\psi} \Gamma^{a} \psi\right) \\
& =\sum_{a=0}^{4}\left(\bar{\psi} \Gamma_{a} \psi\right)\left(\bar{\psi} \Gamma^{a} \psi\right)+\left(\bar{\psi} \Gamma_{5} \psi\right)\left(\bar{\psi} \Gamma^{5} \psi\right) .
\end{aligned}
$$

Remark 6.7 ( $D=5$ super spacetime as homotopy $\Omega S^{2}$-quotient of $\left.D=6\right)$.

Conversely, the total rectangle on the left of (6.11) exhibits, by (6.2), the $D=5$ super-spacetime $\mathbb{R}^{4,1 \mid 8+8}$ as the homotopy $\Omega S^{2}$-quotient of the little string-extended $D=6, \mathcal{N}=2$, super-spacetime:

$$
\mathbb{T}^{4,1 \mid 8+8} \simeq \mathbb{T}^{5,1 \mid 8+8} / / S^{1} \simeq \mathfrak{l t l} \text { string } / / \Omega S^{2} .
$$

(ii) To make this explicit as an $\Omega S^{2}$-quotient in the form of (6.7), we may (co)fibrantly resolve the bottom instead of the right morphism of the rectangle in (6.11). This yields the following:
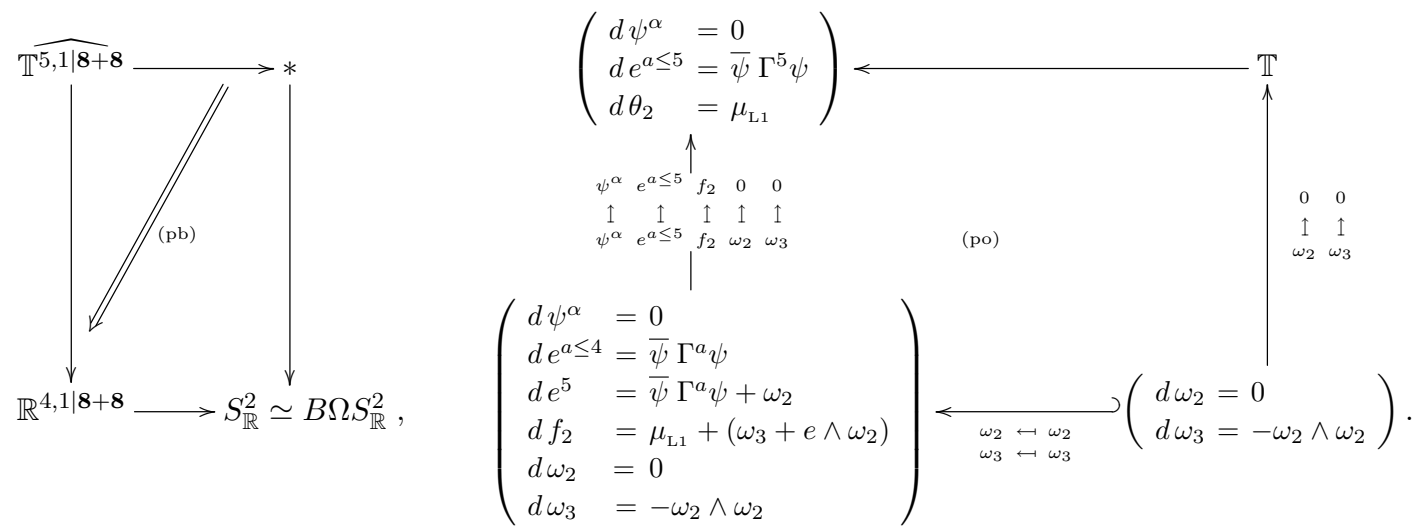

In conclusion, $D=6, \mathcal{N}=(1,1)$, superspacetime extended by its brane content reduces to $D=5$ super-spacetime by enhancing the $\infty$-action by $S^{1}$ to an $\infty$-action by $\Omega S^{2}(6.6)$. 
This provides the motivation to similarly form the homotopy quotient of the superexceptional $\mathcal{N}=(1,0)$ spacetime $\left(\mathbb{T}^{5,1 \mid 8} \times \mathbb{T}^{1}\right)_{\mathrm{ex}_{s}}$ from definition 4.6 not just by the $S_{\mathrm{HW}^{-}}^{1}$ action of flowing along the M-theory circle fiber along the super-exceptional isometry $v_{5}^{\text {ex }} s_{s}$ from definition 4.4, but the left-induced $\Omega S^{2}$-action, via example 6.3 To indicate this, we will write $\Omega S_{\mathrm{HW}}^{2}$ to denote this $\infty$-group with that $\infty$-action understood, hence with the comparison (6.5) specifically being

$$
\Omega S_{\mathrm{HW}}^{2} \longrightarrow S_{\mathrm{HW}}^{1}
$$

Hence:

Definition 6.8 (Homotopy $\Omega S_{\mathrm{HW}}^{2}$-quotient of super-exceptional $\frac{1}{2} \mathrm{M} 5$ along $S_{\mathrm{HW}}^{1}$ ). Write $\left(\mathbb{R}^{5,1 \mid 8} \times \mathbb{R}^{1}\right)_{\mathrm{ex}_{s}} / / \Omega S_{\mathrm{HW}}^{2}$ for the homotopy quotient of the super-exceptional $\frac{1}{2} \mathrm{M} 5$ spacetime (4.6) by the rational $\Omega S_{\mathrm{HW}}^{2}$-action which is left-induced, via example 6.3 , by the rational $S_{\mathrm{HW}}^{1}$-action given by the the super-exceptional $S_{\mathrm{HW}}^{1}$-flow (4.6). Hence, with (6.7), the defining super dgc-algebra (FDA) is that on the right of the following diagram:

$$
\begin{aligned}
& \left(\mathbb{T}^{5,1 \mid \mathbf{8}} \times \mathbb{T}^{1}\right)_{\mathrm{ex}_{s}} \stackrel{q_{\Omega S_{\mathrm{HW}}^{2}}}{\longrightarrow}\left(\mathbb{T}^{5,1 \mid \mathbf{8}} \times \mathbb{T}^{1}\right)_{\mathrm{ex}_{s}} / / \Omega S_{\mathrm{HW}}^{2}
\end{aligned}
$$

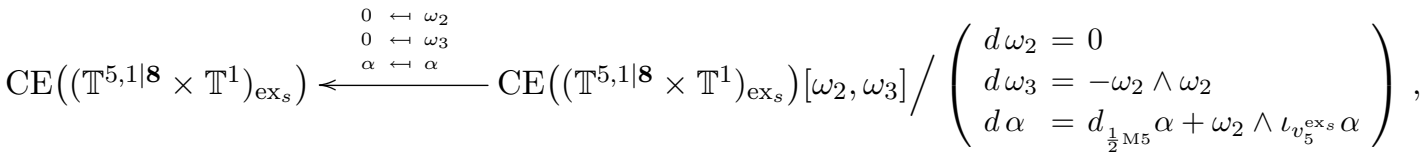

where on the right $\alpha \in \mathrm{CE}\left(\left(\mathbb{T}^{5,1 \mid 8} \times \mathbb{T}^{1}\right)_{\mathrm{ex}_{s}}\right)$ is any element in the CE-algebra of the superexceptional $\frac{1}{2} \mathrm{M} 5$-spacetime (definition 4.6$), \iota_{v_{5}^{\text {ex }}}$ is contraction with the super-exceptional isometry (4.6), and $d_{\frac{1}{2} \mathrm{M} 5}$ now denotes the differential on that algebra, in contrast to the new differential $d$ defined above.

Now we may state and prove the main statement of this section:

Theorem 6.9 (Super-exceptional $\Omega S_{\mathrm{HW}}^{2}$-equivariant M5-cocycle). The super-exceptional Perry-Schwarz Lagrangian $\mathbf{L}_{\mathrm{ex}_{s}}^{\mathrm{PS}}$ (5.4) and the super-exceptional topological Yang-Mills Lagrangian $\mathrm{L}_{\mathrm{ex}}^{\mathrm{tYM}}$ (5.6) are the components that enhance the super-exceptional M5-brane cocycle $\mathbf{d L}_{\mathrm{ex}}^{\mathrm{WZ}}$ (3.12) restricted along the embedding $i_{\mathrm{ex}_{s}}$ of the super-exceptional $\frac{1}{2} \mathrm{M} 5$ spacetime (4.12) to an equivariant cocycle with respect to the $\Omega S_{\mathrm{HW}}^{2}$-action (6.15), hence to a cocycle on the homotopy $\Omega S_{\mathrm{HW}}^{2}$-quotient (6.16) of the super-exceptional $\frac{1}{2} \mathrm{M} 5$-spacetime, as follows:

$$
\left(\left(i_{\mathrm{ex}_{s}}\right)^{*} \mathbf{d L}_{\mathbf{e x}_{s}}^{\mathrm{WZ}}\right)_{/ / \Omega S_{\mathrm{HW}}^{2}}:=\left(\left(i_{\mathrm{ex}_{s}}\right)^{*} \mathbf{d L}_{\mathrm{ex}_{s}}^{\mathrm{WZ}}-\omega_{2} \wedge \mathbf{L}_{\mathrm{ex}_{s}}^{\mathrm{PS}}-\omega_{3} \wedge \mathbf{L}_{\mathrm{ex}_{s}}^{\mathrm{tYM}}\right) \in \mathrm{CE}\left(\left(\mathbb{R}^{5,1 \mid \boldsymbol{8}} \times \mathbb{R}^{1}\right)_{\mathrm{ex}_{s}} / / \Omega S_{\mathrm{HW}}^{2}\right)
$$

in that

(i) this is indeed a cocycle with respect to the differential $d=d_{\frac{1}{2} \mathrm{M} 5}+\omega_{2} \wedge \iota_{v_{5}^{\text {ex }}}+d_{S^{2}}$ from (6.16):

$$
d\left(\left(\left(i_{\mathrm{ex}_{s}}\right)^{*} \mathbf{d L}_{\mathrm{ex}_{s}}^{\mathrm{WZ}}\right)_{/ / \Omega S_{\mathrm{HW}}^{2}}\right)=0 \in \mathrm{CE}\left(\left(\mathbb{R}^{5,1 \mid \mathbf{8}} \times \mathbb{R}^{1}\right)_{\mathrm{ex}_{s}} / / \Omega S_{\mathrm{HW}}^{2}\right) ;
$$


(i) it does enhance the super-exceptional M5-brane cocycle, in that it extends it through the homotopy quotient projection $q_{\Omega S_{\mathrm{HW}}^{2}}(6.16)$ :

$$
\begin{aligned}
& \left(\mathbb{T}^{5,1 \mid \boldsymbol{8}} \times \mathbb{T}^{1}\right)_{\mathrm{ex}_{s}} / / \Omega S_{\mathrm{HW}}^{2} \longleftarrow{ }_{\Omega S_{\mathrm{HW}}^{2}}\left(\mathbb{T}^{5,1 \mid \boldsymbol{8}} \times \mathbb{T}^{1}\right)_{\mathrm{ex}_{s}} \stackrel{i_{\mathrm{ex}_{s}}}{\longrightarrow}\left(\mathbb{T}^{10,1 \mid \mathbf{3 2}}\right)_{\mathrm{ex}_{s}} . \\
& \left(\left(i_{\mathrm{ex}_{s}}\right)^{*} \mathbf{d L}_{\mathrm{ex}_{s}}^{\mathrm{WZ}}\right)_{/ / \Omega S_{\mathrm{HW}}^{2}} \longmapsto\left(i_{\mathrm{ex}_{s}}\right)^{*} \mathbf{d L}_{\mathrm{ex}_{s}}^{\mathrm{WZ}} \longleftrightarrow \mathbf{d L}_{\mathrm{ex}_{s}}^{\mathrm{WZ}}
\end{aligned}
$$

Proof. By lemma 6.4 the first claim equation (6.18), is equivalent to the two statements

1. $d \mathbf{L}_{\mathrm{ex}_{s}}^{\mathrm{PS}}=\iota_{v_{5}}^{\mathrm{ex}_{s}}\left(i_{\mathrm{ex}_{s}}\right)^{*} \mathbf{d} \mathbf{L}_{\mathrm{ex}_{s}}^{\mathrm{WZ}}$.

2. $\iota_{v_{5}}^{\mathrm{ex}_{s}} \mathbf{L}_{\mathrm{ex}_{s}}^{\mathrm{PS}}=\mathbf{L}_{\mathrm{ex}_{s}}^{\mathrm{tYM}}$.

The first of these is the content of proposition 5.9, while the second is lemma 5.6.

The second claim (6.19) is immediate from the nature of the map (6.16).

Before moving on, we record the following further properties of the compactified superexceptional $\frac{1}{2} \mathrm{M} 5$-spacetime:

Proposition 6.10 (Equivariant closedness of tYM). The super-exceptional topological Yang-Mills Lagrangian (definition 5.5) is closed on the homotopy $\Omega S_{\mathrm{HW}}^{2}$-quotient of the super-exceptional $\frac{1}{2} \mathrm{M} 5$-spacetime (definition 6.8):

$$
d \mathbf{L}_{\mathrm{ex}_{s}^{\mathrm{YM}}}^{\mathrm{YM}}=0 \quad \in \mathrm{CE}\left(\left(\left(\mathbb{T}^{5,1 \mid \boldsymbol{8}} \times \mathbb{T}^{1}\right)_{\mathrm{ex}_{s}}\right)_{/ / \Omega S_{\mathrm{HW}}^{2}}\right) .
$$

Proof. By definition (6.16) of the equivariant differential, we have

$$
d \mathbf{L}_{\mathrm{ex}_{s}}^{\mathrm{tYM}}:=d_{\frac{1}{2} \mathrm{M} 5} \mathbf{L}_{\mathrm{ex}_{s}}^{\mathrm{tYM}}+\omega_{2} \wedge \iota_{v_{5}} \mathrm{ex}_{s} \mathbf{L}_{\mathrm{ex}_{s}}^{\mathrm{tYM}} .
$$

By proposition 5.10, both summands here already vanish separately.

Proposition 6.11 (Closedness in the homotopy quotient). On the homotopy $\Omega S_{\mathrm{HW}^{-}}^{-}$ quotient of the super-exceptional $\frac{1}{2} \mathrm{M} 5$-brane spacetime (definition 6.8 ), we have

$$
d e^{5}=\omega_{2} \quad \text { and } \quad d\left(e^{5} \wedge \omega_{2}+\omega_{3}\right)=0 \quad \in \mathrm{CE}\left(\left(\left(\mathbb{T}^{5,1 \mid 8} \times \mathbb{T}^{1}\right)_{\mathrm{ex}_{s}}\right)_{/ / \Omega S_{\mathrm{HW}}^{2}}\right) .
$$

Proof. For the first statement we compute as follows:

$$
\begin{aligned}
& d e^{5}:=\left(d_{\frac{1}{2} \mathrm{M} 5}+\omega_{2} \wedge \iota_{v_{5}^{\text {ex }}}\right) e^{5} \\
& =\underbrace{d_{\frac{1}{2} \mathrm{M} 5} e^{5}}_{=(\overline{P \psi}) \Gamma^{5}(P \psi)=0}+\omega_{2} \wedge \underbrace{\iota_{e_{5}^{\mathrm{ex}}} e^{5}}_{=\delta_{5}^{5}=1}=\omega_{2} .
\end{aligned}
$$

Here the first step is the definition of the equivariant differential (6.16), while the second step uses the definition of $d_{\frac{1}{2}} \mathrm{M} 5$ from (4.11). Then under the first brace we used lemma 4.11, and under the second brace we used the definition (4.6) in proposition 4.4. The second statement is directly implied by the first and by the differential relations $d \omega_{2}=0$ and $d \omega_{3}=-\omega_{2} \wedge \omega_{2}$ from (6.16). 


\section{Super-exceptional M5 Lagrangian from super-exceptional embedding}

Finally we discuss the super-exceptional embedding construction of the M5-brane Lagrangian. We consider the super-exceptional Nambu-Goto Lagrangian for the $\frac{1}{2} \mathrm{M} 5$-brane (definition 7.1) below and prove (corollary 7.4 below) that the sum of super-exceptional Nambu-Goto Lagrangian and the super-exceptional Perry-Schwarz Lagrangian arise as relative trivialization of the super-exceptional M5-brane cocycle along the super-exceptional embedding of the $\frac{1}{2} \mathrm{M} 5$-brane. We go further and consider the $\Omega S_{\mathrm{HW}}^{2}$-equivariant enhancement of this statement, corresponding to KK-compactification to the D4-brane, and prove (theorem 7.3 below) that this corrects the relative trivialization by a summand proportional to the super-exceptional topological Yang-Mills term that constitutes the D4-brane WZ term (remark 7.5 below). While a priori this further summand is exact only after compactification on $S_{\mathrm{HW}}^{1}$, as befits the nature of the $\mathrm{D} 4$ arising form the M5, we observe (remark 7.6 below) that this D4 term, too, does become genuinely exact after a natural completion of the $\Omega S_{\mathrm{HW}}^{2}$-action on the super-exceptional $\frac{1}{2} \mathrm{M} 5$ spacetime.

In direct generalization of the super Nambu-Goto Lagrangian (1.5), we set:

Definition 7.1. The super-exceptional Nambu-Goto Lagrangian for the $\frac{1}{2} \mathrm{M} 5$-brane (remark 4.2) is the super volume form of the $\frac{1}{2}$ M5-locus, hence, with (3.4), the left-invariant completion of the bosonic volume form under translational supersymmetry, hence is the element

$$
\begin{aligned}
\mathbf{L}_{\mathrm{ex}_{s}}^{\mathrm{NG}}:=\operatorname{svol}_{\mathrm{ex}_{s}}^{5+1} & :=e_{0} \wedge e_{1} \wedge e_{2} \wedge e_{3} \wedge e_{4} \wedge e_{5^{\prime}} \\
& =\left(\pi_{\mathrm{ex}_{s}}\right)^{*}\left(e_{0} \wedge e_{1} \wedge e_{2} \wedge e_{3} \wedge e_{4} \wedge e_{5^{\prime}}\right) \in \mathrm{CE}\left(\mathbb{T}^{5,1 \mid 8} \times \mathbb{T}^{1}\right)_{\mathrm{ex}_{s}}
\end{aligned}
$$

in the CE-algebra of the super-exceptional $\frac{1}{2} \mathrm{M} 5$-spacetime (definition 4.6.)

Remark 7.2 ( $S_{\mathrm{B}}^{1}$ vs. $S_{\mathrm{HW}}^{1}$ directions). Beware that the last wedge factor in (7.1) is $e_{5^{\prime}}$ and that $e_{5}$ does not appear (see remark 4.2 for discussion of the 5-5'-plane). Mathematically, this comes out from the MO9-projection in the last step (7.5) in the proof of theorem 7.3 below.

Theorem 7.3 (Full M5-Lagrangian from equivariant super-embedding). The superexceptional $\Omega S_{\mathrm{HW}}^{2}$-equivariant M5-brane cocycle from theorem 6.9 is equal to

$$
\left(\left(i_{\mathrm{ex}_{s}}\right)^{*} \mathbf{d L}_{\mathrm{ex}_{s}}^{\mathrm{WZ}}\right)_{/ / \Omega S_{\mathrm{HW}}^{2}}=d\left(\mathbf{L}_{\mathrm{ex}_{s}}^{\mathrm{NG}}+\mathbf{L}_{\mathrm{ex}_{s}}^{\mathrm{PS}} \wedge e^{5}\right)-\underbrace{\left(e^{5} \wedge \omega_{2}+\omega_{3}\right) \wedge \mathbf{L}_{\mathrm{ex}_{s}}^{\mathrm{tYM}}}_{=e^{5} \wedge d\left(C_{1} \wedge \mathbf{L}_{\mathbf{e x}_{s}}^{\mathrm{YMM}}\right)+\omega_{3} \wedge \mathbf{L}_{\mathbf{e x}_{s}}^{\mathrm{YMM}}},
$$

where under the brace we show an equivalent re-formulation in terms of the element $C_{1}$ from (6.10). 
Proof. We may rewrite (6.17) as follows:

$$
\begin{aligned}
& \left(\left(i_{\mathrm{ex}_{s}}\right)^{*} \mathbf{d L}_{\mathrm{ex}_{s}}^{\mathrm{WZ}}\right)_{/ / \Omega S_{\mathrm{HW}}^{2}} \\
& =\left(i_{\mathrm{ex}_{s}}\right)^{*} \mathbf{d} \mathbf{L}_{\mathrm{ex}_{s}}^{\mathrm{WZ}}-\underbrace{\left(d e^{5}\right)}_{=\omega_{2}} \wedge \mathbf{L}_{\mathrm{ex}_{s}}^{\mathrm{PS}}-\omega_{3} \wedge \mathbf{L}_{\mathrm{ex}_{s}}^{\mathrm{YMM}} \\
& =\left(i_{\mathrm{ex}_{s}}\right)^{*} \mathbf{d L}_{\mathbf{e x}_{s}}^{\mathrm{WZ}}-d\left(e^{5} \wedge \mathbf{L}_{\mathrm{ex}_{s}}^{\mathrm{PS}}\right)-e^{5} \wedge \underbrace{d \mathbf{L}_{\mathrm{ex}_{s}}^{\mathrm{PS}}}-\omega_{3} \wedge \mathbf{L}_{\mathrm{ex}_{s}}^{\mathrm{tYM}} \\
& =\left(d_{\frac{1}{2} \mathrm{M} 5}+\omega_{2} \wedge \iota_{v_{5}}^{\mathrm{ex}_{s}}\right) \mathbf{L}_{\mathrm{ex}_{s}}^{\mathrm{PS}} \\
& =\iota_{v_{5}^{\text {ex }}}\left(i_{\text {ex }_{s}}\right)^{*} \mathbf{d L}_{\mathbf{e x}_{s}}^{\mathrm{WZ}}+\omega^{2} \wedge \mathbf{L}_{\mathbf{e x}_{s}}^{\mathrm{tYM}} \\
& =\underbrace{\left(\mathrm{id}-e^{5} \wedge \iota_{v_{\mathrm{ex}}^{\mathrm{ex}}}\right)}_{=(-)^{\mathrm{horex}_{s}}}\left(\left(i_{\mathrm{ex}_{s}}\right)^{*} \mathbf{d} \mathbf{L}_{\mathrm{ex}_{s}}^{\mathrm{WZ}}\right)+d\left(\mathbf{L}_{\mathrm{ex}_{s}}^{\mathrm{PS}} \wedge e^{5}\right)-\left(\omega_{3}+e^{5} \wedge \omega_{2}\right) \wedge \mathbf{L}_{\mathrm{ex}_{s}}^{\mathrm{tYM}} .
\end{aligned}
$$

Here the first line is the definition (6.17) with the observation (6.21) inserted, as shown under the brace. Then, in the first step, we use that the differential is a derivation of bi-degree (1, even) and under the brace we unwind the definition of the equivariant differential (6.16) and then used proposition 5.9 and lemma 5.6. In the last step we collect terms and identify under the brace the super-exceptional horizontal projection from definition 4.5. This means we are now reduced to showing that the first summand in the last line of (7.3) is

$$
\left(\left(i_{\mathrm{ex}_{s}}\right)^{*} \mathbf{d L}_{\mathrm{ex}_{s}}^{\mathrm{WZ}}\right)^{\text {hor }_{\mathrm{ex}}}=d \mathbf{L}_{\mathrm{ex}_{s}}^{\mathrm{NG}} .
$$

We compute as follows:

$$
\begin{aligned}
\left(\left(i_{\mathrm{ex}_{s}}\right)^{*} \mathbf{d L}_{\mathrm{ex}_{s}}^{\mathrm{WZ}}\right)^{\mathrm{hor}_{\mathrm{ex}_{s}}=} & \left(\mathrm{id}-e^{5} \wedge \iota_{v_{5}^{\mathrm{ex}_{s}}}\right)\left(i_{\mathrm{ex}_{s}}\right)^{*}\left(\left(\pi_{\mathrm{ex}_{s}}\right)^{*} \mu_{\mathrm{M}_{5}}+\frac{1}{2} H_{\mathrm{ex}_{s}} \wedge d H_{\mathrm{ex}_{s}}\right) \\
= & \left(\mathrm{id}-e^{5} \wedge \iota_{v_{5}^{\mathrm{ex}_{s}}}\right)\left(i_{\mathrm{ex}_{s}}\right)^{*}\left(\pi_{\mathrm{ex}_{s}}\right)^{*} \mu_{\mathrm{M} 5} \\
& +\frac{1}{2}\left(\mathrm{id}-e^{5} \wedge \iota_{v_{5}^{\mathrm{ex}_{s}}}\right)\left(\left(i_{\mathrm{ex}_{s}}\right)^{*} H_{\mathrm{ex}_{s}}\right) \wedge\left(\left(i_{\mathrm{ex}_{s}}\right)^{*} d H_{\mathrm{ex}_{s}}\right) \\
= & \frac{1}{5 !} \sum_{a_{i} \in\left\{0,1,2,3,4,5^{\prime}\right\}}\left(\overline{(P \psi)} \Gamma_{a_{1} \cdots a_{5}}(P \psi)\right) \wedge e_{a_{0}} \wedge \cdots \wedge e_{a_{5}} \\
& +\underbrace{\frac{1}{2}\left(\mathrm{id}-e^{5} \wedge \iota_{v_{5}^{\mathrm{ex}_{s}}}\right)\left(\left(i_{\mathrm{ex}_{s}}\right)^{*} H_{\mathrm{ex}_{s}}\right) \wedge\left(e^{5} \wedge \iota_{v_{5}}^{\operatorname{ex}_{s}}\left(i_{\mathrm{ex}_{s}}\right)^{*} d H_{\mathrm{ex}_{s}}\right)}_{=0} \\
= & d\left(e_{0} \wedge e_{1} \wedge e_{2} \wedge e_{3} \wedge e_{4} \wedge e_{5^{\prime}}\right) .
\end{aligned}
$$

Here the first step is unwinding the definitions. The second step is multiplying out and using, in the second summand, the fact that pullback is an algebra homomorphism. The third step observes that the first term is just those summands of $\mu_{\mathrm{M} 5}$ (1.2) whose indices are along the $\frac{1}{2} \mathrm{M} 5$-locus, hence in $\left\{0,1,2,3,4,5^{\prime}\right\}$, while in the second term we realize the presence of the projection $e^{5} \wedge \iota_{v_{5}}^{\mathrm{ex}_{s}}$ according to lemma 4.12, in view of $d H_{\mathrm{ex}_{s}}=\left(\pi_{\mathrm{ex}_{s}}\right)^{*} \mu_{\mathrm{M} 2}$ from (3.5). With the projection operator up front, this makes the second term vanish, as shown under the brace. The last step is [69, lemma 6.9]. This establishes the first line in $(7.2)$. 
Finally, to see equality to the expression shown in (7.2) under the brace, observe that

$$
d e^{5}=d\left(d x^{5}+C_{1}\right)=d C_{1}
$$

by (6.10). Using this, the first summand over the brace in (7.2) becomes

$$
\begin{aligned}
e^{5} \wedge \omega_{2} \wedge \mathbf{L}_{\mathrm{ex}_{s}}^{\mathrm{tYM}} & =e^{5} \wedge\left(d e^{5}\right) \wedge \mathbf{L}_{\mathrm{ex}_{s}}^{\mathrm{tYM}} \\
& =e^{5} \wedge\left(d C_{1}\right) \wedge \mathbf{L}_{\mathrm{ex}_{s}}^{\mathrm{YM}} \\
& =e^{5} \wedge d\left(C_{1} \wedge \mathbf{L}_{\mathrm{ex}_{s}}^{\mathrm{YMM}}\right)
\end{aligned}
$$

where the first step is (6.21), the second step is (7.6) and the third step is (6.20). This, therefore, establishes also the identification under the brace in (7.2).

Corollary 7.4 (M5-Lagrangian is relative trivialization along super-exceptional embedding). The super-exceptional M5-brane cocycle $\mathbf{d L}_{\mathrm{ex}_{s}}^{\mathrm{WZ}}$ (definition 3.8) becomes exact when restricted along the super-exceptional embedding $\left(i_{\mathrm{ex}_{s}}\right)$ of the $\frac{1}{2} \mathrm{M} 5$-brane (lemma 4.8), trivialized there by the sum of the super-exceptional Nambu-Goto Lagrangian $\mathbf{L}_{\mathrm{ex}_{s}}^{\mathrm{NG}}$ (definition 7.1) and the super-exceptional Perry-Schwarz Lagrangian $\mathbf{L}_{\mathrm{ex}_{s}}^{\mathrm{PS}}$ (definition 5.2):

$$
\left(i_{\mathrm{ex}_{s}}\right)^{*} \mathbf{d L}_{\mathrm{ex}_{s}}^{\mathrm{WZ}}=d\left(\mathbf{L}_{\mathrm{ex}_{s}}^{\mathrm{WZ}}+\mathbf{L}_{\mathrm{ex}_{s}}^{\mathrm{PS}} \wedge e^{5}\right) .
$$

Proof. The statement is the first component (the one independent of the equivariance generators $\omega_{2}$ and $\omega_{3}$ ) of the equivariant statement in theorem 7.3. More formally, equation (7.7) is the pullback of (2.6) along the homotopy quotient homomorphism $q_{\Omega S_{\mathrm{HW}}^{2}}$ (6.16).

Remark 7.5 (Dimensional reduction to WZ-term of D4-brane). Upon compactification on $S_{\mathrm{HW}}^{1}$, the last summand of (7.2) manifestly gives the WZ-term

$$
\mathbf{L}_{\mathrm{D} 4}^{\mathrm{WZ}}=C_{1} \wedge F \wedge F
$$

of the D4-brane ([27, (7.4)], [2, (51)], [30, 6.1]; see [39, 4.3], [44, 4]). From the point of view of the Yang-Mills theory on the brane, this identifies $C_{1}$ with the theta-angle (e.g. [79, (3.1)]), matching the last summand in (7.11) below.

Remark 7.6 (Exact $\Omega S_{\mathrm{HW}}^{2}$-equivariant super-exceptional M5-Lagrangians). Recall that the first summand in (5.8) is exact, implying the super-exceptional embedding construction before compactification (corollary 7.4), while the second summand in (5.8)

$$
\left(e^{5} \wedge \omega_{2}+\omega_{3}\right) \wedge\left(F_{\mathrm{ex}_{s}} \wedge F_{\mathrm{ex}_{s}}\right) \quad \in \mathrm{CE}\left(\left(\left(\mathbb{T}^{5,1 \mid 8} \times \mathbb{T}^{1}\right)_{\mathrm{ex}_{s}}\right)_{/ / \Omega S_{\mathrm{HW}}^{2}}\right)
$$

becomes exact only after dimensional reduction, implying the super-exceptional embedding construction of the D4 WZ-term (remark 7.5). It is therefore natural to ask for a pullback of the situation to a richer extended super-spacetime on which also the second summand (7.8), and hence the full $\Omega S_{\mathrm{HW}}^{2}$-equivariant super-embedded super-exceptional M5-brane cocycle from (5.8), become exact, $\Omega S_{\mathrm{HW}^{-}}^{2}$-equivariantly. Since (7.8) is the wedge product of two equivariantly closed terms, by proposition 6.10 and proposition 6.11 , there are two canonical 
possibilities here, by enforcing trivialization of the first or the second factor. We will now briefly comment on both of these:

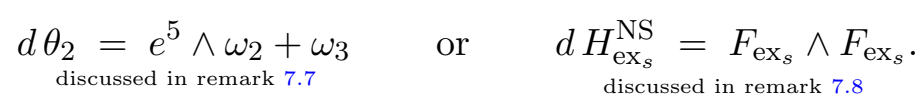

Remark 7.7 (The generator $\theta_{2}$ trivializing the little-string super-cocycle). In the formula (6.14) for the $D=5, \mathcal{N}=2$, super-spacetime regarded as an $\Omega S^{2}$-quotient of $D=6$, $\mathcal{N}=(1,1)$, super-spacetime, there appears a further generator $\theta_{2}$ in degree $(2$, even), whose differential trivializes the little-string super-cocycle

$$
d \theta_{2}=\omega_{3}+e^{5} \wedge \omega_{2}=\mu_{\mathrm{L} 1} .
$$

From comparison with the super-cocycles for the ordinary critical string in $D=10$ and its D-branes (see $[39,4.3],[44,4]$ ), we note that $\theta_{2}$ is the universal gauge field flux 2-form on D-branes for the little-string. However, there is no analog of the generator $\theta_{2}$ in the formula (6.16) for the super-exceptional $\Omega S_{\mathrm{HW}}^{2}$-quotient of the super-exceptional $\frac{1}{2} \mathrm{M} 5$-locus from definition 6.8. But if we consider the Cartesian product of the super-exceptional $\frac{1}{2} \mathrm{M} 5$ spacetime with the classifying space $B S^{1}$ of a gauge field, then an $\Omega S^{2}$-action on this larger space generally contains, on top of the part left-induced from an $S^{1}$-action (example 6.3), precisely the extra structure of (7.9):

$$
\begin{aligned}
& \operatorname{CE}\left(\mathfrak{l}\left(\left(\mathbb{T}^{5,1} \times \mathbb{T}^{1}\right)_{\mathrm{ex}_{s}} \times B S^{1}\right)_{/ / \Omega S_{\mathrm{HW}}^{2}}\right) \\
& =\mathrm{CE}\left(\left(\left(\mathbb{T}^{5,1} \times \mathbb{T}^{1}\right)_{\mathrm{ex}_{s}}\right)_{/ / \Omega S_{\mathrm{HW}}^{2}}\right)\left[\theta_{2}\right] /\left(d \theta_{2}=e^{5} \wedge \omega_{2}+\omega_{3}\right) .
\end{aligned}
$$

On this larger space, equation (7.2) for the trivialization of the M5-brane cocycle completes to an $\Omega S_{\mathrm{HW}^{-}}^{2}$ equivariant trivialization:

$$
\begin{aligned}
&\left(\left(i_{\mathrm{ex}_{s}}\right)^{*} \mathbf{d L}_{\mathrm{ex}_{s}}^{\mathrm{WZ}}\right)_{/ / \Omega S_{\mathrm{HW}}^{2}}=d\left(\mathbf{L}_{\mathrm{ex}_{s}}^{\mathrm{NG}}+\mathbf{L}_{\mathrm{ex}_{s}}^{\mathrm{PS}} \wedge e^{5}-\theta_{2} \wedge \mathbf{L}_{\mathrm{ex}_{s}}^{\mathrm{tYM}}\right) \\
& \text { on }\left(\left(\mathbb{T}^{5,1} \times \mathbb{T}^{1}\right)_{\mathrm{ex}_{s}} \times B S^{1}\right)_{/ / \Omega S_{\mathrm{HW}}^{2}} .
\end{aligned}
$$

Remark 7.8 (The heterotic 5-brane). The heterotic super-exceptional $\frac{1}{2} \mathrm{M} 5$-spacetime $\left(\mathbb{T}^{5,1 \mid 16} \times \mathbb{T}^{1}\right)_{\mathrm{ex}_{s}}^{\text {het }}$ is the homotopy fiber of the wedge square of the super-exceptional 2flux (5.3) on the super-exceptional $\frac{1}{2} \mathrm{M} 5$-spacetime (definition 4.6)
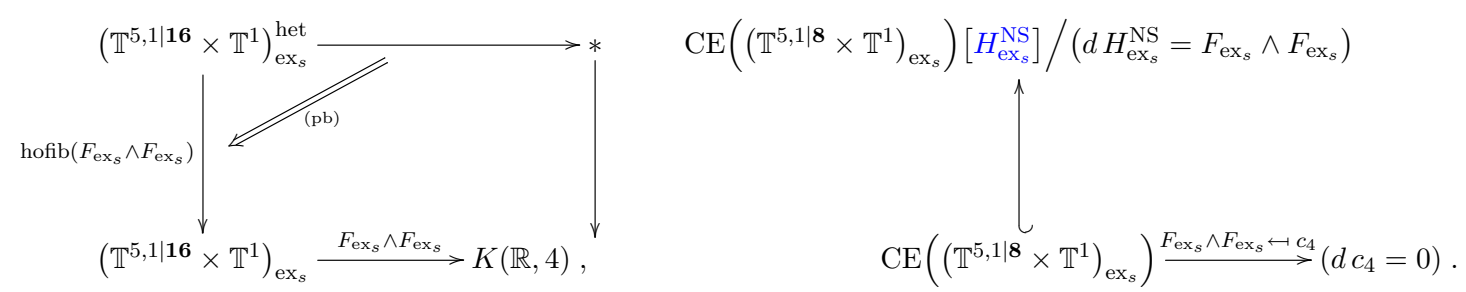

Then on its super-exceptional $\Omega S_{\mathrm{HW}^{-}}^{2}$-compactification as in definition 6.8 the second summand (7.8) and with it the full $\Omega S_{\mathrm{HW}^{-}}^{2}$-equivariant super-exceptional M5-brane cocycle (7.2) 
becomes exact

$$
\left(\left(i_{\mathrm{ex}_{s}}\right)^{*} \mathbf{d L}_{\mathrm{ex}_{s}}^{\mathrm{WZ}}\right)_{/ / \Omega S_{\mathrm{HW}}^{2}}=d(\mathbf{L}_{\mathrm{ex}_{s}}^{\mathrm{NG}}+\mathbf{L}_{\mathrm{ex}_{s}}^{\mathrm{PS}} \wedge e^{5}+\underbrace{\frac{1}{2}\left(e^{5} \wedge \omega_{2}+\omega_{3}\right) \wedge H_{\mathrm{ex}_{s}}^{\mathrm{NS}}}_{=: \mathbf{L}_{\mathrm{NS} 5}^{\mathrm{WZ}}}) .
$$

The new term $\mathbf{L}_{\mathrm{NS} 5}^{\mathrm{WZ}}$ in the Lagrangian that appears this way has the form of the WZ-term for the heterotic NS5-brane [78, (1.5)].

In summary, we thus arrive at the picture shown in (1.7).

\section{Outlook}

In closing, we briefly comment on a few interconnections, issues to be addressed in the future, and some loose ends.

Combining local super-exceptional geometry with global topology. The discussion in this article focuses on the situation of vanishing bosonic 4-flux, keeping only the super-components of the 4-flux, being the M2-brane cocycle $\mu_{\mathrm{M} 2}$ (1.2). We had discussed the opposite case of pure bosonic flux in [48]. In that case, the subtlety is all in the global topological structure of the Hopf-Wess-Zumino term controlled by Cohomotopy cohomology theory [47, 48], while here the subtlety is all in the local differential structure of the Perry-Schwarz Lagrangian, hence of 5d super Yang-Mills plus KK-modes:

\begin{tabular}{|c|c|c|}
\hline \multicolumn{2}{|c|}{ Single M5-brane sigma-model } \\
\hline Aspect & Global topological & Local differential \\
\hline \multirow{2}{*}{ Lagrangian term } & Hopf-Wess-Zumino & Perry-Schwarz $=5 \mathrm{~d}$ Yang-Mills + KK \\
& $\&$ 5d top. Yang-Mills \\
\hline \multirow{2}{*}{ Controlled by } & twisted & $\Omega S^{2}$-equivariant \\
& Cohomotopy & super-exceptional geometry \\
\hline Discussed in & {$[48]$} & section 6, section 7 \\
\hline
\end{tabular}

In a full picture of the M5-brane sigma-model, both the super-exceptional local geometry and the cohomotopical global structure are to be combined. This will be discussed elsewhere.

Non-abelian gauge enhancement. While we have only discussed abelian gauge fields here, their appearance (by the construction in section 5 , section 7 ) via the M2/M5 supercocycle $\mu_{\mathrm{M} 2 / \mathrm{M} 5}$ (1.2) means that the super-cohomotopical gauge enhancement mechanism found in [19] applies. Together with the constraints of half-integral flux quantization and tadpole cancellation, which we demonstrate in [47] and [104], respectively, to follow from C-field charge quantization in full Cohomotopy (8.1), this should generate the expected types of non-abelian gauge fields, both for heterotic M-theory as well as for coincident 5 -branes, in the form discussed in [40, 41, 100, 101]. While the details remain to be worked out, this opens up the possibility of a concrete strategy for systematically deriving the non-abelian $\mathrm{D}=6 \mathrm{M} 5$-brane theory from first principles. 
A proposal for the gauge sector of a non-abelian M5-brane Lagrangian based on the higher structures of $[40,41,101]$ has recently been made in $[95,96]$, but the details are notoriously subtle and hard to get right by an educated Ansatz, see [94]. With the above strategy this Ansatz might eventually be connected to a systematic derivation.

Covariant enhancement. The constructions in this article relate to the non-covariant M5-brane Lagrangian of [1, 90, 105], (following [61]), but not manifestly to the covariant formulation of $[12,88,89]$.

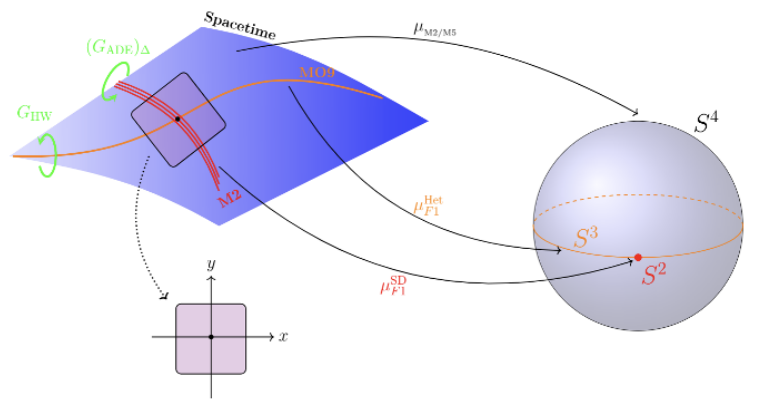

However, as explained in [69, pp. 6-7], in the vein of understanding supergravity as super Cartan geometry $[36,80]$, we are to think of the super-exceptional Minkowski spacetimes considered here, such as the super-exceptional $\frac{1}{2} \mathrm{M} 5$-spacetime illustrated in (4.2), as being the infinitesimal moving frames on a super-exceptional curved Cartan geometry. Furthermore, the covariant form of this geometry is to be obtained systematically by actually moving the frames, subject only to the condition of vanishing super-torsion, which, by [23, 65] (see [26, section 2.4]), is equivalently the condition that the equations of motion of $11 \mathrm{~d}$ supergravity hold.

Thus super-Cartan geometry provides a systematic and precise mechanism for globalizing/covariantizing all the local brane constructions given here, as well as the related constructions in $[19,69]$, reviewed in [46]. While this provides the algorithm, it still has to be run, the details still have to be worked out elsewhere.

Necessity of the $\frac{1}{2}$ M5 configuration? The string theory folklore claims (e.g. [33]) that there are two ways of geometrically engineering $D=6 \mathcal{N}=(1,0)$ theories, one of them being the $\frac{1}{2} \mathrm{M} 5$-brane configuration of remark 4.2. Since our rigorous construction confirms this expectation, it would be interesting to precisely classify this - on a mathematical basis - and possibly determine further available choices for the construction. This needs further thinking. At this point, we may at least highlight precisely where the spinor projections of definition 4.1, which define the $\frac{1}{2}$ M5-locus, enter our proofs:

1. The assumption that the spinor 1-forms are fixed under $\boldsymbol{\Gamma}_{6789}$ is what makes the superexceptional lift $v_{a}^{\mathrm{ex}}$ of the isometric flow along the compactification circle exist: it is used in the last line of (4.9) in the proof of proposition 4.4. There is at least one other brane configuration where an analogous construction works, namely the M-wave inside the MO9 (remark 4.10). But there could be more possible variants. 
2. The assumptions that spinor 1 -forms are fixed under $\boldsymbol{\Gamma}_{5}$ is what implies the technical lemma 4.12. This lemma appears crucially in various of the following proofs leading up to and including the main theorem 7.3, notably it is used in both (5.9) and (5.10) proving proposition 5.9 and then again in (7.5) proving theorem 7.3.

This makes it seem at least unlikely that this assumption could be removed while still retaining the result of a super-exceptional embedding construction.

DBI corrections in $\boldsymbol{\alpha}^{\prime}$ and super-exceptional 5-form. In our analysis of the superexceptional Perry-Schwarz Lagrangian in section 5, we have restricted to the special case that the value of the 5 -index tensor $e_{a_{1} \cdots a_{5}}$ on super-exceptional spacetime vanishes. The formulas (3.5) and (3.6) show that, without this assumption, the super-exceptional PerrySchwarz Lagrangian picks up further correction terms. At the same time, we have studied here the expected DBI corrections to the brane Lagrangian starting at quartic order in the field strength $F$ [49] (see [112]). It is natural to conjecture that these two effects are related, but this needs more investigation.

AKSZ sigma-model description. The article [6] discusses the local differential structure of the M5 Hopf-Wess-Zumino term in view of exceptional geometry from the point of view of AKSZ sigma-models. The development seems complementary to ours here, but it would be interesting to see if there is a connection.

\section{Acknowledgments}

The authors would like to thank Alex Arvanitakis, Igor Bandos, and Dmitri Sorokin for useful comments on the first draft. For the final stages of this project, H. S. gratefully acknowledges: "This work was performed in part at Aspen Center for Physics, which is supported by National Science Foundation grant PHY-1607611. This work was partially supported by a grant from the Simons Foundation."

Open Access. This article is distributed under the terms of the Creative Commons Attribution License (CC-BY 4.0), which permits any use, distribution and reproduction in any medium, provided the original author(s) and source are credited.

\section{References}

[1] M. Aganagic, J. Park, C. Popescu and J.H. Schwarz, World volume action of the M-theory five-brane, Nucl. Phys. B 496 (1997) 191 [hep-th/9701166] [INSPIRE].

[2] M. Aganagic, J. Park, C. Popescu and J.H. Schwarz, Dual D-brane actions, Nucl. Phys. B 496 (1997) 215 [hep-th/9702133] [INSPIRE].

[3] O. Aharony and A. Hanany, Branes, superpotentials and superconformal fixed points, Nucl. Phys. B 504 (1997) 239 [hep-th/9704170] [INSPIRE].

[4] L. Andrianopoli, R. D'Auria and L. Ravera, Hidden gauge structure of supersymmetric free differential algebras, JHEP 08 (2016) 095 [arXiv: 1606.07328] [INSPIRE]. 
[5] F. Apruzzi and M. Fazzi, $A d S_{7} / C F T_{6}$ with orientifolds, JHEP 01 (2018) 124 [arXiv: 1712.03235] [INSPIRE].

[6] A.S. Arvanitakis, Brane Wess-Zumino terms from AKSZ and exceptional generalised geometry as an $L_{\infty}$-algebroid, arXiv:1804.07303 [INSPIRE].

[7] J.J. Atick, A. Dhar and B. Ratra, Superspace formulation of ten-dimensional $N=1$ supergravity coupled to $N=1$ Super-Yang-Mills theory, Phys. Rev. D 33 (1986) 2824 [INSPIRE].

[8] P. van Baal, An introduction to topological Yang-Mills theory, Acta Phys. Polon. B 21 (1990) 73.

[9] I. Bandos, Exceptional field theories, superparticles in an enlarged $11 D$ superspace and higher spin theories, Nucl. Phys. B 925 (2017) 28 [arXiv:1612.01321] [InSPIRE].

[10] I.A. Bandos et al., On the underlying gauge group structure of $D=11$ supergravity, Phys. Lett. B 596 (2004) 145 [hep-th/0406020] [INSPIRE].

[11] I.A. Bandos, D.P. Sorokin and D. Volkov, On the generalized action principle for superstrings and supermembranes, Phys. Lett. B 352 (1995) 269 [hep-th/9502141] [INSPIRE].

[12] I.A. Bandos et al., Covariant action for the superfive-brane of M-theory, Phys. Rev. Lett. 78 (1997) 4332 [hep-th/9701149] [INSPIRE].

[13] I.A. Bandos et al., Superstrings and supermembranes in the doubly supersymmetric geometrical approach, Nucl. Phys. B 446 (1995) 79 [hep-th/9501113] [InSPIRE].

[14] L. Baulieu and I. Singer, Topological Yang-Mills symmetry, Nucl. Phys. Proc. Suppl. B 5 (1988) 12.

[15] K. Becker, M. Becker and A. Strominger, Five-branes, membranes and nonperturbative string theory, Nucl. Phys. B 456 (1995) 130 [hep-th/9507158] [INSPIRE].

[16] E. Bergshoeff, E. Sezgin and P.K. Townsend, Supermembranes and eleven-dimensional supergravity, Phys. Lett. B 189 (1987) 75 [INSPIRE].

[17] L. Bonora et al., Anomaly free supergravity and Super-Yang-Mills theories in ten-dimensions, Nucl. Phys. B 296 (1988) 877 [INSPIRE].

[18] V. Braunack-Mayer, Rational parametrised stable homotopy theory, Ph.D. thesis, Zürich, Switzerland (2018).

[19] V. Braunack-Mayer, H. Sati and U. Schreiber, Gauge enhancement of super M-branes via parametrized stable homotopy theory, Commun. Math. Phys. 371 (2019) 197 [arXiv: 1806.01115] [INSPIRE].

$[20]$ D. Butter, H. Samtleben and E. Sezgin, $E_{7(7)}$ exceptional field theory in superspace, JHEP 01 (2019) 087 [arXiv:1811.00038] [inSPIRE].

[21] S. Cabrera, A. Hanany and M. Sperling, Magnetic quivers, Higgs branches and $6 d$ $N=(1,0)$ theories, JHEP 06 (2019) 071 [arXiv: 1904.12293] [INSPIRE].

[22] L. Castellani, R. D'Auria and P. Fré, Supergravity and Superstrings - A geometric perspective, World Scientific, Singapore (1991).

[23] A. Candiello and K. Lechner, Duality in supergravity theories, Nucl. Phys. B 412 (1994) 479 [hep-th/9309143] [INSPIRE]. 
[24] M. Cederwall, Fundamental issues in extended geometry, talk given at the $8^{\text {th }}$ Mathematical Physics Meeting, August 24-31, Belgrade, Serbia (2014).

[25] M. Cederwall, J. Edlund and A. Karlsson, Exceptional geometry and tensor fields, JHEP 07 (2013) 028 [arXiv: 1302.6736] [inSPIRE].

[26] M. Cederwall, U. Gran, B.E.W. Nilsson and D. Tsimpis, Supersymmetric corrections to eleven-dimensional supergravity, JHEP 05 (2005) 052 [hep-th/0409107] [INSPIRE].

[27] M. Cederwall et al., The Dirichlet super p-branes in ten-dimensional type IIA and IIB supergravity, Nucl. Phys. B 490 (1997) 179 [hep-th/9611159] [INSPIRE].

[28] E. Cremmer and B. Julia, The SO(8) supergravity, Nucl. Phys. B 159 (1979) 141 [inSPIRE].

[29] E. Cremmer, Supergravities in 5 dimensions, in Superspace and supergravity, S.W. Hawking and M. Rocek eds., Cambridge University Press, Cambridge U.K. (1981).

[30] C. Chryssomalakos, J.A. de Azcarraga, J.M. Izquierdo and J.C. Perez Bueno, The Geometry of branes and extended superspaces, Nucl. Phys. B 567 (2000) 293 [hep-th/9904137] [INSPIRE].

[31] A. Dasgupta, H. Nicolai and J. Plefka, An introduction to the quantum supermembrane, Grav. Cosmol. 8 (2002) 1 [hep-th/0201182] [INSPIRE].

[32] R. D'Auria and P. Fré, Geometric supergravity in D = 11 and its hidden supergroup, Nucl. Phys. B 201 (1982) 101, ncatlab.org/nlab/files/GeometricSupergravity.pdf.

[33] M. Del Zotto, J.J. Heckman, A. Tomasiello and C. Vafa, 6d conformal Matter, JHEP 02 (2015) 054 [arXiv: 1407.6359] [inSPIRE].

[34] B. de Wit, J. Hoppe and H. Nicolai, On the quantum mechanics of supermembranes, Nucl. Phys. B 305 (1988) 545 [InSPIRE].

[35] M.R. Douglas, On D = 5 super Yang-Mills theory and (2,0) theory, JHEP 02 (2011) 011 [arXiv: 1012.2880] [INSPIRE].

[36] M. Egeileh and F. El Chami, Some remarks on the geometry of superspace supergravity, J. Geom. Phys. 62 (2012) 53 [INSPIRE].

[37] J. Evslin and H. Sati, SUSY versus $E_{8}$ gauge theory in eleven-dimensions, JHEP 05 (2003) 048 [hep-th/0210090] [INSPIRE].

[38] O. de Felice, Flux backgrounds and exceptional generalised geometry, Ph.D. thesis, LPTHE, Paris, France (2018), arXiv:1808.04225 [INSPIRE].

[39] D. Fiorenza, H. Sati and U. Schreiber, Super Lie n-algebra extensions, higher WZW models and super p-branes with tensor multiplet fields, Int. J. Geom. Meth. Mod. Phys. 12 (2014) 1550018 [arXiv: 1308.5264] [INSPIRE].

[40] D. Fiorenza, H. Sati and U. Schreiber, The E $E_{8}$ Moduli 3-stack of the C-field in M-theory, Commun. Math. Phys. 333 (2015) 117 [arXiv: 1202.2455] [INSPIRE].

[41] D. Fiorenza, H. Sati and U. Schreiber, Multiple M5-branes, string 2-connections and $7 d$ non-Abelian Chern-Simons theory, Adv. Theor. Math. Phys. 18 (2014) 229 [arXiv:1201.5277] [INSPIRE].

[42] D. Fiorenza, H. Sati and U. Schreiber, The Wess-Zumino-Witten term of the M5-brane and differential cohomotopy, J. Math. Phys. 56 (2015) 102301 [arXiv:1506.07557] [InSPIRE]. 
[43] D. Fiorenza, H. Sati and U. Schreiber, Rational sphere valued supercocycles in M-theory and type IIA string theory, J. Geom. Phys. 114 (2017) 91 [arXiv:1606.03206] [INSPIRE].

[44] D. Fiorenza, H. Sati and U. Schreiber, T-duality from super Lie $n$-algebra cocycles for super p-branes, Adv. Theor. Math. Phys. 22 (2018) 1209 [arXiv:1611.06536] [InSPIRE].

[45] D. Fiorenza, H. Sati and U. Schreiber, Higher T-duality of super M-branes, arXiv: 1803.05634 [INSPIRE].

[46] D. Fiorenza, H. Sati and U. Schreiber, The rational higher structure of M-theory, Fortsch. Phys. 67 (2019) 1910017 [arXiv: 1903.02834] [INSPIRE].

[47] D. Fiorenza, H. Sati and U. Schreiber, Twisted cohomotopy implies M-theory anomaly cancellation on 8-manifolds, arXiv:1904.10207 [INSPIRE].

[48] D. Fiorenza, H. Sati and U. Schreiber, Twisted cohomotopy implies level quantization of the full 6d Wess-Zumino term of the M5-brane, arXiv:1906.07417 [INSPIRE].

[49] E.S. Fradkin and A.A. Tseytlin, Nonlinear electrodynamics from quantized strings, Phys. Lett. 163B (1985) 123 [INSPIRE].

[50] D. Gaiotto and A. Tomasiello, Holography for $(1,0)$ theories in six dimensions, JHEP 12 (2014) 003 [arXiv: 1404.0711] [INSPIRE].

[51] E. Gorbatov et al., On heterotic orbifolds, M-theory and type-I-prime brane engineering, JHEP 05 (2002) 015 [hep-th/0108135] [INSPIRE].

[52] M.B. Green and J.H. Schwarz, Covariant description of superstrings, Phys. Lett. 136B (1984) 367 [INSPIRE].

[53] P. Griffiths and J. Morgan, Rational homotopy theory and differential forms, Progress in Mathematics vol. 16, Birkhaüser, Switzerland (2013).

[54] A. Güijosa, QCD, with strings attached, Int. J. Mod. Phys. E 25 (2016) 1630006 [arXiv: 1611.07472] [INSPIRE].

[55] V.W. Guillemin and S. Sternberg, Supersymmetry and equivariant de Rham theory, Springer, Germany (1999).

[56] A. Hanany and A. Zaffaroni, Branes and six-dimensional supersymmetric theories, Nucl. Phys. B 529 (1998) 180 [hep-th/9712145] [INSPIRE].

[57] A. Hanany and A. Zaffaroni, Issues on orientifolds: on the brane construction of gauge theories with $\mathrm{SO}(2 N)$ global symmetry, JHEP 07 (1999) 009 [hep-th/9903242] [INSPIRE].

[58] J.A. Harvey and G.W. Moore, Superpotentials and membrane instantons, hep-th/9907026 [INSPIRE].

[59] H. Hayashi et al., More on 5d descriptions of 6d SCFTs, JHEP 10 (2016) 126 [arXiv: 1512.08239] [INSPIRE].

[60] J.J. Heckman and T. Rudelius, Top down approach to 6D SCFTs, J. Phys. A 52 (2019) 093001 [arXiv: 1805.06467] [INSPIRE].

[61] M. Henneaux and C. Teitelboim, Dynamics of chiral (self-dual) p-forms, Phys. Lett. B 206 (1988) 650 .

[62] K. Hess, Rational homotopy theory: a brief introduction, in Interactions between homotopy theory and algebra, L.L. Avramov ed., Contemporary Mathematics volume 436, AMS, U.S.A. (2007), math.AT/0604626. 
[63] P. Hořava and E. Witten, Heterotic and type-I string dynamics from eleven-dimensions, Nucl. Phys. B 460 (1996) 506 [hep-th/9510209] [INSPIRE].

[64] P. Hořava and E. Witten, Eleven-dimensional supergravity on a manifold with boundary, Nucl. Phys. B 475 (1996) 94 [hep-th/9603142] [INSPIRE].

[65] P.S. Howe, Weyl superspace, Phys. Lett. B 415 (1997) 149 [hep-th/9707184] [INSPIRE].

[66] P.S. Howe and E. Sezgin, D =11, p=5, Phys. Lett. B 394 (1997) 62 [hep-th/9611008] [INSPIRE].

[67] P.S. Howe, E. Sezgin and P.C. West, Covariant field equations of the M-theory five-brane, Phys. Lett. B 399 (1997) 49 [hep-th/9702008] [INSPIRE].

[68] P.S. Howe and E. Sezgin, The supermembrane revisited, Class. Quant. Grav. 22 (2005) 2167 [hep-th/0412245] [INSPIRE].

[69] J. Huerta, H. Sati and U. Schreiber, Real ADE-equivariant (co)homotopy and Super M-branes, Commun. Math. Phys. 371 (2019) 425 [arXiv: 1805.05987] [INSPIRE].

[70] C.M. Hull, Generalised geometry for M-theory, JHEP 07 (2007) 079 [hep-th/0701203] [INSPIRE].

[71] C.M. Hull and P.K. Townsend, Unity of superstring dualities, Nucl. Phys. B 438 (1995) 109 [hep-th/9410167] [INSPIRE].

[72] B. Julia, Group disintegrations, talk given at the Nuffield Gravity Workshop, JUne 22-July 12, Cambridge, U.K. (1980).

[73] B. Jurčo, C. Sämann, U. Schreiber and M. Wolf, Higher structures in M-Theory, Fortsch. Phys. 67 (2019) 1910001 [arXiv: 1903.02807] [INSPIRE].

[74] V. Kaplunovsky, J. Sonnenschein, S. Theisen and S. Yankielowicz, On the duality between perturbative heterotic orbifolds and M-theory on $T^{4} / Z_{N}$, Nucl. Phys. B 590 (2000) 123 [hep-th/9912144] [INSPIRE].

[75] K. Koepsell, H. Nicolai and H. Samtleben, An exceptional geometry for $D=11$ supergravity?, Class. Quant. Grav. 17 (2000) 3689 [hep-th/0006034] [InSPIRE].

[76] N. Lambert, C. Papageorgakis and M. Schmidt-Sommerfeld, M5-branes, D4-branes and quantum 5D Super-Yang-Mills, JHEP 01 (2011) 083 [arXiv:1012.2882] [INSPIRE].

[77] N. Lambert, M-branes: lessons from M2's and Hopes for M5's, Fortsch. Phys. 67 (2019) 1910011 [arXiv: 1903.02825] [INSPIRE].

[78] K. Lechner, Quantum properties of the heterotic five-brane, Phys. Lett. B 693 (2010) 323 [arXiv: 1005.5719] [INSPIRE].

[79] S.-W. Li, The theta-dependent Yang-Mills theory at finite temperature in a holographic description, Chin. Phys. C 44 (2020) 013103 [arXiv:1907.10277] [INSPIRE].

[80] J. Lott, The geometry of supergravity torsion constraints, Comm. Math. Phys. 133 (1990) 563 [math/0108125].

[81] V. Mathai and D.G. Quillen, Superconnections, Thom classes and equivariant differential forms, Topology 25 (1986) 85 [INSPIRE].

[82] E. Meinrenken, Equivariant cohomology and the Cartan model, Encyclopedia of Mathematical Physics, Elsevier, The Netherlands (2006). 
[83] G. Moore, Applications of the six-dimensional $(2,0)$ theories to Physical Mathematics, Felix Klein lectures, Bonn, Germany (2012).

[84] G. Moore, Physical mathematics and the future, talk given at Strings 2014, June 23-27, Princeton, U.S.A. (2014).

[85] A. Neveu and J. Schwarz, Factorizable dual model of pions, Nucl. Phys. B 31 (1971) 86.

[86] T. Nikolaus, U. Schreiber and D. Stevenson, Principal $\infty$-bundles - General theory, J. Homotopy Rel. Struc. 10 (2015) 749 [arXiv: 1207.0248].

[87] P. Pires Pacheco and D. Waldram, M-theory, exceptional generalised geometry and superpotentials, JHEP 09 (2008) 123 [arXiv:0804.1362] [INSPIRE].

[88] P. Pasti, D.P. Sorokin and M. Tonin, On Lorentz invariant actions for chiral $p$ forms, Phys. Rev. D 55 (1997) 6292 [hep-th/9611100] [INSPIRE].

[89] P. Pasti, D.P. Sorokin and M. Tonin, Covariant action for a $D=11$ five-brane with the chiral field, Phys. Lett. B 398 (1997) 41 [hep-th/9701037] [INSPIRE].

[90] M. Perry and J.H. Schwarz, Interacting chiral gauge fields in six-dimensions and Born-Infeld theory, Nucl. Phys. B 489 (1997) 47 [hep-th/9611065] [InSPIRE].

[91] D. Quillen, Rational homotopy theory, Annals Math. 90 (1969) 205.

[92] P. Ramond, Dual theory for free fermions, Phys. Rev. D 3 (1971) 2415 [inSPIRE].

[93] A. Rebhan, The Witten-Sakai-Sugimoto model: A brief review and some recent results, EPJ Web Conf. 95 (2015) 02005 [arXiv: 1410.8858] [INSPIRE].

[94] C. Sämann, Higher Structures, Self-Dual Strings and 6d Superconformal Field Theories, in the proceedings of Durham Symposium, Higher Structures in M-theory, August 12-18, Durham, U.K. (2019), arXiv: 1903.02888 [INSPIRE].

[95] C. Sämann and L. Schmidt, Towards an M5-brane model I: a 6d superconformal field theory, J. Math. Phys. 59 (2018) 043502 [arXiv:1712.06623] [INSPIRE].

[96] C. Sämann and L. Schmidt, Towards an M5-brane model II: metric string structures, arXiv: 1908.08086 [INSPIRE].

[97] T. Sakai and S. Sugimoto, Low energy hadron physics in holographic QCD, Prog. Theor. Phys. 113 (2005) 843 [hep-th/0412141] [InSPIRE].

[98] T. Sakai and S. Sugimoto, More on a holographic dual of QCD, Prog. Theor. Phys. 114 (2005) 1083 [hep-th/0507073] [INSPIRE].

[99] M. Sakaguchi, IIB Branes and new space-time superalgebras, JHEP 04 (2000) 019 [hep-th/9909143] [INSPIRE].

[100] H. Sati, Geometric and topological structures related to M-branes, Proc. Symp. Pure Math. 81 (2010) 181 [arXiv: 1001.5020] [INSPIRE].

[101] H. Sati, Geometric and topological structures related to M-branes II: Twisted String and String ${ }^{c}$ structures, J. Austral. Math. Soc. 90 (2011) 93 [arXiv:1007.5419] [INSPIRE].

[102] H. Sati, Framed M-branes, corners and topological invariants, J. Math. Phys. 59 (2018) 062304 [arXiv: 1310.1060] [INSPIRE].

[103] H. Sati and U. Schreiber, Higher T-duality in M-theory via local supersymmetry, Phys. Lett. B 781 (2018) 694 [arXiv: 1805.00233] [INSPIRE]. 
[104] H. Sati and U. Schreiber, Equivariant Cohomotopy implies tadpole anomaly cancellation, in preparation.

[105] J.H. Schwarz, Coupling a selfdual tensor to gravity in six-dimensions, Phys. Lett. B 395 (1997) 191 [hep-th/9701008] [INSPIRE].

[106] D.P. Sorokin, Superbranes and superembeddings, Phys. Rept. 329 (2000) 1 [hep-th/9906142] [INSPIRE].

[107] D.P. Sorokin, Introduction to the superembedding description of superbranes, AIP Conf. Proc. 589 (2001) 98 [hep-th/0105102] [INSPIRE].

[108] D.P. Sorokin, V.I. Tkach and D.V. Volkov, Superparticles, twistors and Siegel symmetry, Mod. Phys. Lett. A 4 (1989) 901 [inSPIRE].

[109] S. Sugimoto, Skyrmion and String theory, in The multifaceted Skyrmion, M. Rho and I. Zahed eds., World Scientific, Singapore (2016).

[110] D. Sullivan, Infinitesimal computations in topology, Pub. Math. IHES 47 (1977) 269.

[111] P.K. Townsend, $M$ theory from its superalgebra, NATO Sci. Ser. C 520 (1999) 141 [hep-th/9712004] [INSPIRE].

[112] A.A. Tseytlin, Born-Infeld action, supersymmetry and string theory, hep-th/9908105 [INSPIRE].

[113] P. van Nieuwenhuizen, Free graded differential superalgebras, in the proceedings of Group theoretical methods in physics, V.V. Dodonov and V.I. Man'ko eds., Springer, Germany (1990).

[114] S. Vaula, On the underlying $E_{11}$ symmetry of the $D=11$ free differential Algebra, JHEP 03 (2007) 010 [hep-th/0612130] [INSPIRE].

[115] P.C. West, $E_{11}, \mathrm{SL}(32)$ and central charges, Phys. Lett. B 575 (2003) 333 [hep-th/0307098] [INSPIRE].

[116] P. West, Generalised geometry, eleven dimensions and E11, JHEP 02 (2012) 018 [arXiv:1111.1642] [INSPIRE].

[117] E. Witten, Twistor-like transform in ten-dimensions, Nucl. Phys. B 266 (1986) 245 [INSPIRE].

[118] E. Witten, Solutions of four-dimensional field theories via M-theory, Nucl. Phys. B 500 (1997) 3 [hep-th/9703166] [INSPIRE].

[119] E. Witten, Anti-de Sitter space, thermal phase transition and confinement in gauge theories, Adv. Theor. Math. Phys. 2 (1998) 505 [hep-th/9803131] [InSPIRE].

[120] E. Witten, Conformal Field Theory In Four And Six Dimensions, in the proceedings of Topology, geometry and quantum field theory. Symposium in the honour of the $60^{\text {th }}$ birthday of Graeme Segal, June 24-29, Oxford, U.K. (2002), arXiv:0712.0157 [INSPIRE]. 